\title{
\begin{tabular}{l|l} 
Mitraries & DSpace@MIT
\end{tabular}
}

\author{
MIT Open Access Articles
}

\section{OVERTONE AND MULTI-MODE RR LYRAE STARS IN THE GLOBULAR CLUSTER M3}

The MIT Faculty has made this article openly available. Please share how this access benefits you. Your story matters.

Citation: Jurcsik, J., P. Smitola, G. Hajdu, A. Sodor, J. Nuspl, K. Kolenberg, G. Furesz, et al. “OVERTONE AND MULTI-MODE RR LYRAE STARS IN THE GLOBULAR CLUSTER M3.” The Astrophysical Journal Supplement Series 219, no. 2 (August 1, 2015): 25. (C) 2015 The American Astronomical Society

As Published: http://dx.doi.org/10.1088/0067-0049/219/2/25

Persistent URL: http://hdl.handle.net/1721.1/100032

Version: Final published version: final published article, as it appeared in a journal, conference proceedings, or other formally published context

Terms of Use: Article is made available in accordance with the publisher's policy and may be subject to US copyright law. Please refer to the publisher's site for terms of use. 


\title{
OVERTONE AND MULTI-MODE RR LYRAE STARS IN THE GLOBULAR CLUSTER M3
}

\author{
J. Jurcsik ${ }^{1}$, P. Smitola ${ }^{1}$, G. Hajdu ${ }^{2,3}$, Á. Sódor ${ }^{1}$, J. Nuspl ${ }^{1}$, K. Kolenderg ${ }^{4,5}$, G. Fúrész ${ }^{6}$, A. Moór ${ }^{1}$, E. Kun ${ }^{7,8}$, A. PÁl $^{1}$, \\ J. Bakos ${ }^{1}$, J. Kelemen ${ }^{1}$, T. Kovács ${ }^{1}$, L. Kriskovics ${ }^{1}$, K. Sárneczky ${ }^{1}$, T. Szalai ${ }^{9}$, A. Szing ${ }^{10}$, and K. Vida ${ }^{1}$ \\ ${ }^{1}$ Konkoly Observatory of the Hungarian Academy of Sciences, H-1525 Budapest P.O. Box 67, Hungary; jurcsik@konkoly.hu \\ ${ }^{2}$ Instituto de Astrofísica, Pontificia Universidad Católica de Chile, Av. Vicuña Mackenna 4860, 782-0436 Macul, Santiago, Chile \\ ${ }^{3}$ Instituto Milenio de Astrofísica, Santiago, Chile \\ ${ }^{4}$ Institute of Astronomy, KU Leuven, Celestijnenlaan 200D, B-3001 Heverlee, Belgium \\ ${ }^{5}$ Harvard-Smithsonian Center for Astrophysics, 60 Garden Street, Cambridge MA 02138, USA \\ ${ }^{6}$ MIT Kavli Institute for Astrophysics and Space Research 77 Mass Ave 37-515, Cambridge, MA, 02139, USA \\ ${ }^{7}$ Department of Theoretical Physics, University of Szeged, H-6720 Szeged, Tisza Lajos krt 84-86, Hungary \\ ${ }^{8}$ Department of Experimental Physics and Astronomical Observatory, University of Szeged, H-6720 Szeged, Dóm tér 9, Hungary \\ ${ }^{9}$ Department of Optics and Quantum Electronics, University of Szeged, H-6720 Szeged, Dóm tér 9, Hungary \\ ${ }^{0}$ Baja Observatory, University of Szeged, 6500 Baja, KT: 766, Hungary \\ Received 2015 March 20; accepted 2015 April 22; published 2015 August 11
}

\begin{abstract}
The overtone and multi-mode RR Lyrae stars in the globular cluster M3 are studied using a 200 day long, $B, V$, and $I_{\mathrm{C}}$ time-series photometry obtained in $2012.70 \%$ of the 52 overtone variables observed show some kind of multi-periodicity (with additional frequency at $f_{0.61}=f_{10} / 0.61$ frequency ratio, Blazhko effect, double/multi-mode pulsation, and period doubling). A signal at the 0.587 frequency ratio to the fundamental-mode frequency is detected in the double-mode star, V13, which may be identified as the second radial overtone mode. If this mode identification is correct, than V13 is the first RR Lyrae star showing triple-mode pulsation of the first three radial modes. Either the Blazhko effect or the $f_{0.61}$ frequency (or both of these phenomena) appears in seven doublemode stars. The $P_{1 \mathrm{O}} / P_{\mathrm{F}}$ period ratio of RRd stars showing the Blazhko effect are anomalous. A displacement of the main frequency component at the fundamental mode with the value of modulation frequency (or its half), is detected in three Blazhko RRd stars that are parallel with the appearance of the overtone-mode pulsation. The $f_{0.61}$ frequency appears in RRc stars that lie at the blue side of the double-mode region and in RRd stars, raising the suspicion that its occurrence may be connected to double-mode pulsation. The changes of the Blazhko and doublemode properties of the stars are also reviewed using the recent and archive photometric data.
\end{abstract}

Key words: globular clusters: individual (M3) - stars: horizontal-branch - stars: oscillations - stars: variables: RR Lyrae

Supporting material: machine-readable tables

\section{INTRODUCTION}

Studies of cluster variables (RR Lyrae stars) date back to the end of the 19th century. The large number of the variables discovered in galactic and extragalactic globular clusters (GCs) provided decisive support to specify the main physical properties and the evolutionary status of RR Lyrae stars. Because of the large distance $(d>6 \mathrm{kpc})$ and the relatively small absolute dimension (total radius, $R_{t}<50 \mathrm{pc}$ ) of GCs, the apparent magnitudes of the cluster members reflect the distribution of their absolute magnitudes with 1\%-2\% accuracy (supposing that the foreground/intra-cluster reddening is homogeneous). The advantage that the relative values of the physical properties of GC stars can be determined more accurately than for any other stellar population makes GC studies very prosperous.

While numerous studies of fundamental-mode RR Lyrae stars (RRab) have appeared year after year in the literature, this is not the case for the overtone (RRc) variables. As RRc stars are relatively rare objects and the similarity of their light curves to W UMa binaries makes even the target selection dubious, only a few field RRc stars have been the subject of detailed photometric and/or spectroscopic studies. Photometric data of GCs showed that about 30\% of their RR Lyrae populations are overtone and double-mode (RRd) pulsators (Clement et al. 2001). RRd stars have always been a focus of interest because of their astrophysical importance, and the discovery of peculiar features in the pulsations of RRc stars (Moskalik 2014; Moskalik et al. 2015; Netzel et al. 2015; Smolec et al. 2015a, $2015 \mathrm{~b}$, and references therein) has recently attracted special attention to overtone RR Lyrae stars. Higher order or multiple pulsations, together with modulation or period doubling (PD) make these stars unique targets of theoretical investigations. The complex and interrelated dynamical features give strong constraints on the nonlinear physics governing their variability, and pose a new challenge to further theoretical work, providing a test bed for improved models.

Thanks to the new data secured by space missions (CoRoT, Kepler; Szabó et al. 2014; Szabó 2014) and ground-based projects (Jurcsik et al. 2009; Le Borgne et al. 2012), a lot of new results were published in the recent years concerning the Blazhko effect; the amplitude and/or phase modulation of the pulsation of RR Lyrae stars. Despite these observational results and the accompanying theoretical efforts, revealing the genuine origin of the phenomenon has still eluded explanation. The modulation was detected in about $50 \%$ of the RRab stars (Jurcsik et al. 2009; Benkő et al. 2014), but no reliable Blazhko statistics exist for overtone variables.

One of the most intriguing new questions concerning overtone RR Lyrae and Cepheid stars is the recent discovery of a peculiar frequency at the 0.61 frequency ratio in many of these variables (Soszyński et al. 2010; Dziembowski 2012; Moskalik et al. 2015, and references therein). The interpretation 
Table 1

$B, V, I_{\mathrm{C}} \mathrm{CCD}$ Observations of $\mathrm{M} 3$ Variables Published in this Paper

\begin{tabular}{lccc}
\hline \hline Star $^{\mathrm{a}}$ & HJD-2400000 $^{\mathrm{b}}$ & mag & Band \\
\hline V012 & 55935.64720 & 16.088 & B \\
V012 & 55937.69026 & 15.583 & B \\
V012 & 55937.70074 & 15.636 & B \\
\hline
\end{tabular}

Notes.

a Identification according to the catalog of Samus et al. (2009).

b The HJDs represent the times of mid-exposure.

(This table is available in its entirety in machine-readable form.)

Table 2

Flux Data of M3 Variables with Uncertain Magnitudes in the Reference Frame in $B$ Band

\begin{tabular}{lcc}
\hline \hline Star $^{\mathrm{a}}$ & HJD $^{2} 2400000^{\mathrm{b}}$ & Flux $^{\mathrm{c}}$ \\
\hline V129 & 55935.64720 & 15583.9 \\
V129 & 55937.69026 & 16631.5 \\
V129 & 55937.70074 & 15274.6 \\
\hline
\end{tabular}

Notes.

a Identification according to the catalog of Samus et al. (2009).

${ }^{b}$ The HJDs represent the times of mid-exposure.

${ }^{c}$ B-band flux differences of ISIS photometry (Alard 2000).

(This table is available in its entirety in machine-readable form.)

of this strange signal is hard in the recent theoretical framework because of its unknown excitation mechanism.

M3 is a GC with a large RR Lyrae population, which was previously investigated in many photometric studies. However, even the most extended CCD observations (e.g., Kaluzny et al. 1998, K98; Corwin \& Carney 2001, C01; Hartman et al. 2005, H05; Benkó et al. 2006, B06; and Jurcsik et al. 2012a, J12) were not long and dense enough and/or used only one filter, therefore our knowledge on the multi-mode and Blazhko properties of M3 variables is still incomplete. The present article gives an in-depth study of the overtone and double-mode RR Lyrae stars of M3, with full details on their modulations and multi-mode properties, as well as on the longterm changes of the light-curve variations based on an extended new multicolor time-series photometry of the cluster and archive data. Some of the interesting features of the Blazhko and doublemode properties of overtone variables using the new data set published here were already published in Jurcsik et al. (2014).

\section{THE PHOTOMETRIC DATA: OBSERVATIONS, DATA PROCESSING AND ANALYSIS}

An extended photometric campaign was organized to observe M3 with the Konkoly Observatory $90 / 60 \mathrm{~cm}$ Schmidt telescope (Piszkéstetô) equipped with a 4096×4096 Apogee CCD camera and Bessel $B V I_{\mathrm{C}}$ filters. About 1000 measurements were obtained in each band between 2012 January and July. The standard calibration processes of the CCD frames were performed using IRAF/CCDRED tasks. ${ }^{11}$ The data were

\footnotetext{
${ }^{11}$ IRAF is distributed by the National Optical Astronomy Observatories, which are operated by the Association of Universities for Research in Astronomy, Inc., under cooperative agreement with the National Science Foundation.
}

not corrected for the nonlinearity (less than $2 \%$ ) of the CCD system. As a consequence of the nonlinearity, linear combinations of independent frequencies may appear in the data as artifacts with mmag amplitude, and the measured amplitudes may be uncertain up to $1 \%-2 \%$. Both aperture (IRAF/ DAOPHOT/PHOT) and Image Subtraction Method (ISIS, Alard 2000) photometry of the variables were performed. The light curves were transformed to the standard $B V I_{\mathrm{C}}$ system using 23 bright, photometrically stable standard stars, covering a wide range in color, from the list of Stetson (2000).

A time-series photometry of 52 overtone and double-mode RR Lyrae was obtained. The flux-magnitude transformation of the results of the ISIS photometry needs accurate magnitudes on the reference frames. The point-spread function photometry (using IRAF/DAOPHOT/ALLSTAR) on the reference frames was performed very circumspectly; the neighboring stars of each variable were checked on SDSS images. ${ }^{12}$ The $B V I_{C}$ calibration of the light curves was accurate for 28 stars (Figure 1), but it remained uncertain because of crowding for 24 variables. Aside from the pulsation period, additional properties of the light curves were detected in some of these non-calibrated flux time series. Therefore, the magnitude transformation of the $B$-filter flux curves of these 24 stars was also performed (Figure 2) with reference magnitude values that yielded mean magnitudes matching the horizontal-branch magnitude of the cluster, which was just for the visualization of the light curves. The time-series photometry obtained using the image subtraction method is sensitive to the contamination of only nearby variables. The frequencies of close variables in the neighborhood appeared with low amplitude in the spectrum of some of the variables with non-calibrated light curves, but these signals were removed from the data. No flux curve could be derived for five overtone variables (V152, V155, V178, V266, and V269) because their ISIS photometry gave unreliable results due to blending with another variable.

The CCD time series of the variables are available as machine-readable tables; Tables 1 and 2 give samples of the magnitude calibrated $B, V, I_{\mathrm{C}}$, and the $B$-filter flux data regarding their form and content. These data are referred to as D12 in the paper.

The light curves were analyzed using the program packages MUFRAN (Kolláth 1990) and LCfit (Sódor 2012). Independent frequencies were selected in successive prewhitening steps if their amplitudes were larger than $4 \sigma$ in at least two bands. For linear-combination terms, a less strict criterion was used.

The long-term behavior and the changes of some special properties of the stars were checked using the CCD data published in $\mathrm{K} 98, \mathrm{C} 01, \mathrm{H} 05, \mathrm{~B} 06$, and $\mathrm{J} 12$, and in the photographic data collected in J12. All the previous photometric data are magnitude homogenized and merged.

\section{THE LIGHT CURVES, VARIABLE TYPES}

Tables 3 and 4 summarize the main characteristics of the light curves of the overtone and the double- (multi-)mode variables, respectively, based on the D12 data. Pulsation mode, period, magnitude-averaged, and intensity-averaged $B, V, I_{\mathrm{C}}$ brightnesses, amplitudes, and remarks on any additional property are listed in the columns. The amplitude ratio of the radial modes in the B-band is also given for non-Blazhko RRd

\footnotetext{
${ }^{12}$ http://www.sdss.org/
} 
10
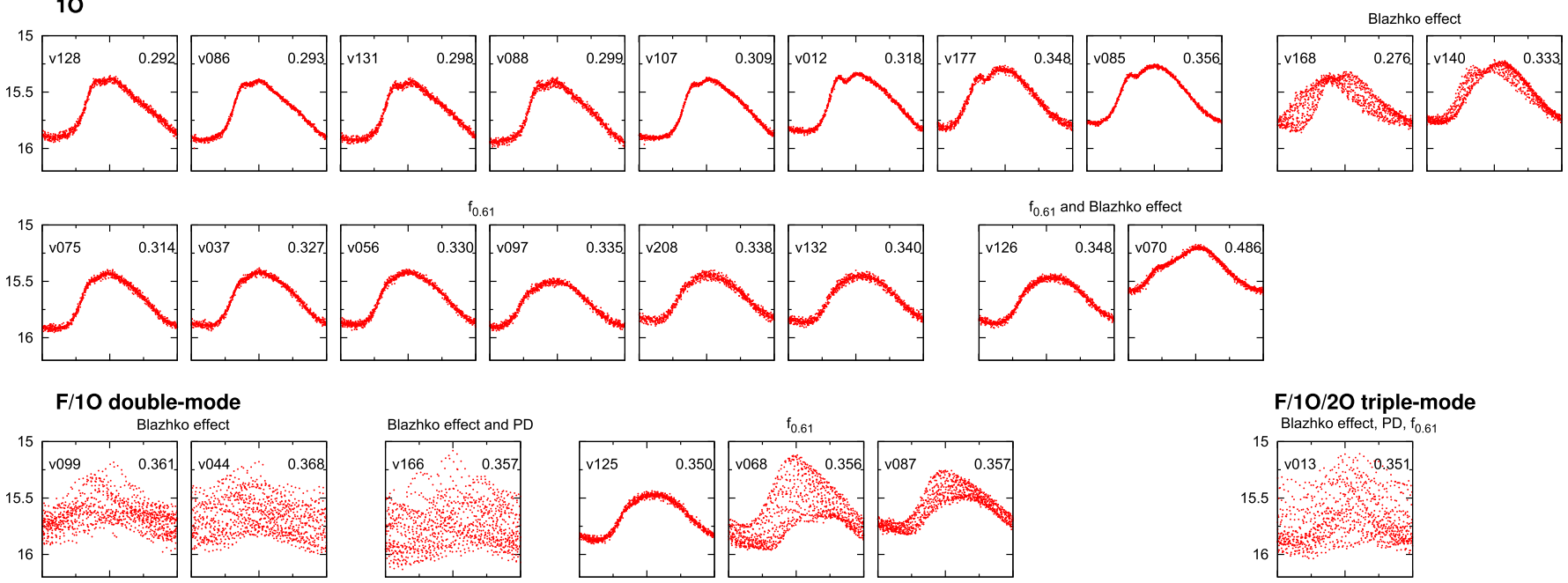

20
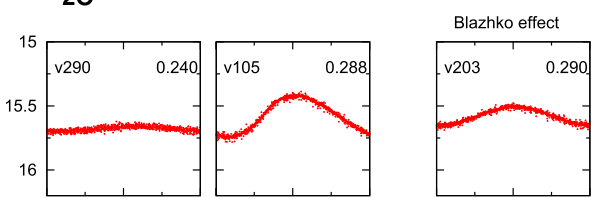

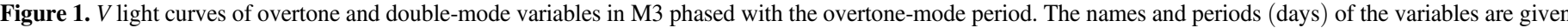
in the top-left- and top-right-side of each panel.

10
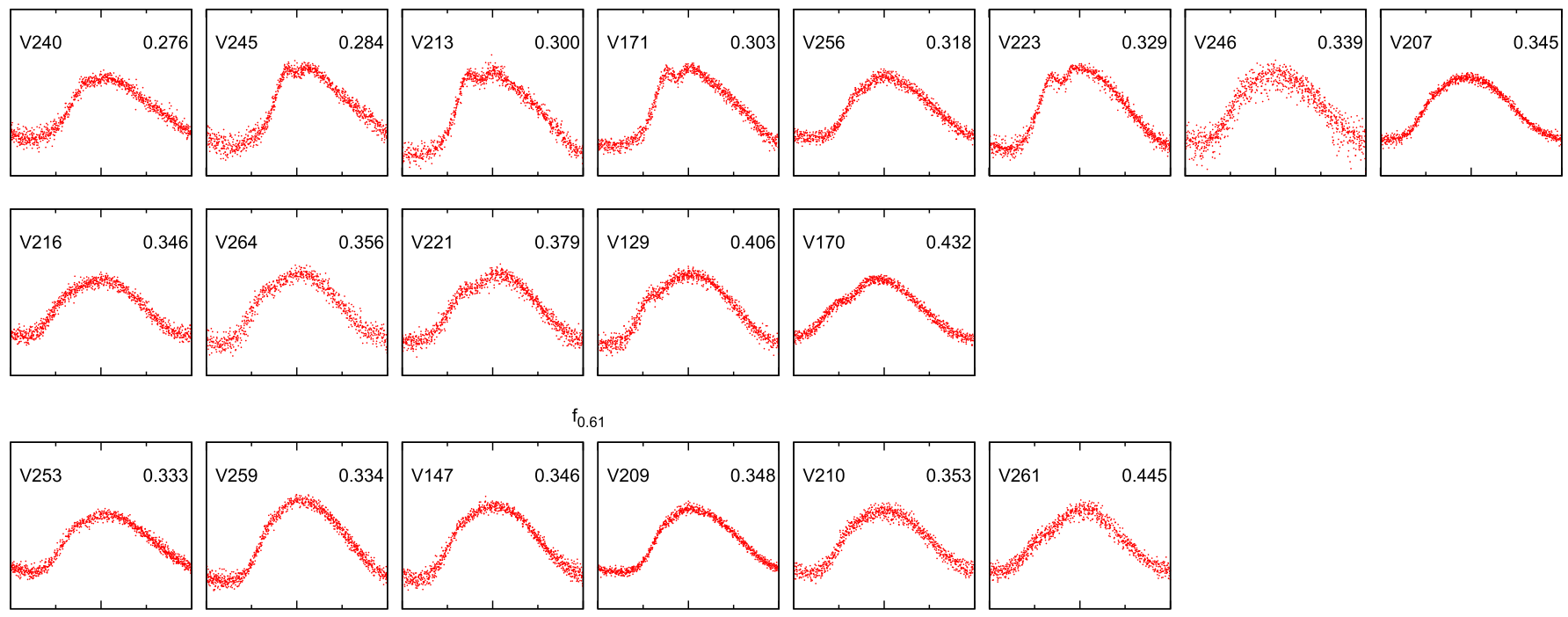

F/10 double mode

20(?)
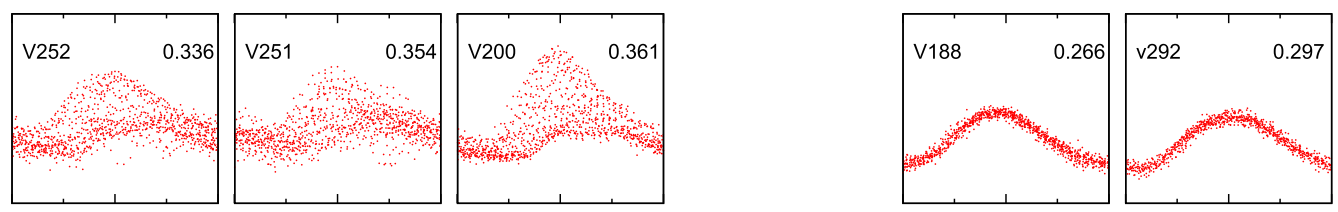

Figure 2. Non-calibrated $B$-filter light curves of overtone and double-mode variables in M3 phased with the overtone-mode period. The ISIS fluxes of the variables with uncertain magnitudes on the reference frame have been transformed to magnitudes matching the mean brightness of the horizontal branch, however, the amplitudes of these light curves are uncertain.

stars in Table 4. The stars for which the photometry is contaminated by signals from nearby (within $6^{\prime \prime}$ ) variable stars are denoted by asterisk in Tables 3 and 4 .
The panels in Figure 3 show the location of the stars on the $V$-period, $A(B)$-period, $V-(B-V)$, and $(V-I)-(B-V)$ planes. The double-mode (red triangle and diamond), first 
Table 3

Summary of the Photometric Properties of the Overtone-mode Variables in M3

\begin{tabular}{|c|c|c|c|c|c|c|c|c|c|c|c|c|c|}
\hline Star $^{\mathrm{a}}$ & Mode $^{b}$ & $\begin{array}{l}\text { Period } \\
\text { (days) }\end{array}$ & $\begin{array}{c}\langle B\rangle^{\mathrm{c}} \\
(\mathrm{mag})\end{array}$ & $\begin{array}{c}(B)^{\mathrm{c}} \\
(\mathrm{mag})\end{array}$ & $\begin{array}{c}\langle V\rangle \\
(\mathrm{mag})\end{array}$ & $\begin{array}{c}(V) \\
(\mathrm{mag})\end{array}$ & $\begin{array}{c}\left\langle I_{\mathrm{C}}\right\rangle \\
(\mathrm{mag})\end{array}$ & $\begin{array}{c}\left(I_{\mathrm{C}}\right) \\
(\mathrm{mag})\end{array}$ & $\begin{array}{c}A_{B}{ }^{\mathrm{d}} \\
(\mathrm{mag})\end{array}$ & $\begin{array}{c}A_{V}^{\mathrm{d}} \\
(\mathrm{mag})\end{array}$ & $\begin{array}{l}A_{I_{\mathrm{C}}}{ }^{\mathrm{d}} \\
(\mathrm{mag})\end{array}$ & \multicolumn{2}{|c|}{$\begin{array}{c}\text { Additional } \\
\text { Feature }^{\mathrm{e}}\end{array}$} \\
\hline V12 & 10 & 0.31786 & 15.829 & 15.802 & 15.600 & 15.584 & 15.264 & 15.258 & 0.65 & 0.51 & 0.32 & $\ldots$ & $\ldots$ \\
\hline V37 & 10 & 0.32663 & 15.909 & 15.885 & 15.664 & 15.650 & 15.311 & 15.306 & 0.64 & 0.48 & 0.30 & $f_{0.61}$ & $\cdots$ \\
\hline V56 & 10 & 0.32960 & 15.908 & 15.884 & 15.657 & 15.643 & 15.278 & 15.272 & 0.62 & 0.47 & 0.29 & $f_{0.61}$ & $\cdots$ \\
\hline V70 & 10 & 0.48607 & 15.691 & 15.677 & 15.398 & 15.391 & 14.963 & 14.960 & $0.53-0.49$ & $0.40-0.38$ & $0.25-0.24$ & $f_{0.61}$ & B1 \\
\hline V75 & 10 & 0.31408 & 15.924 & 15.899 & 15.686 & 15.672 & 15.306 & 15.300 & 0.63 & 0.49 & 0.30 & $f_{0.61}$ & $\cdots$ \\
\hline V85 & 10 & 0.35581 & 15.746 & 15.720 & 15.521 & 15.505 & 15.190 & 15.184 & 0.65 & 0.51 & 0.32 & $\ldots$ & $\ldots$ \\
\hline V86 & 10 & 0.29266 & 15.914 & 15.886 & 15.679 & 15.663 & 15.367 & 15.361 & 0.69 & 0.54 & 0.33 & $\ldots$ & $\ldots$ \\
\hline V88 & 10 & 0.29875 & 15.923 & 15.893 & 15.693 & 15.675 & 15.369 & 15.362 & 0.70 & 0.54 & 0.32 & $\cdots$ & $\cdots$ \\
\hline V97 & 10 & 0.33493 & 15.973 & 15.955 & 15.699 & 15.689 & 15.329 & 15.325 & 0.53 & 0.40 & 0.26 & $f_{0.61}$ & $\cdots$ \\
\hline V105 & 20 & 0.28775 & 15.787 & 15.776 & 15.581 & 15.576 & 15.299 & 15.297 & 0.42 & 0.32 & 0.20 & $\ldots$ & $\ldots$ \\
\hline V107 & 10 & 0.30903 & 15.896 & 15.868 & 15.663 & 15.646 & 15.327 & 15.320 & 0.68 & 0.52 & 0.33 & $\ldots$ & $\ldots$ \\
\hline V126 & 10 & 0.34841 & 15.935 & 15.917 & 15.665 & 15.655 & 15.284 & 15.280 & $0.60-0.53$ & $0.44-0.40$ & $0.30-0.26$ & $f_{0.61}$ & B1 \\
\hline V128 & 10 & 0.29204 & 15.894 & 15.866 & 15.657 & 15.641 & $\ldots$ & $\ldots$ & 0.69 & 0.52 & $\ldots$ & $\ldots$ & $\ldots$ \\
\hline V129 & 10 & 0.40604 & $\cdots$ & $\cdots$ & $\cdots$ & $\cdots$ & $\cdots$ & $\ldots$ & $\cdots$ & $\cdots$ & $\cdots$ & $\cdots$ & $\cdots$ \\
\hline V131 & 10 & 0.29769 & 15.909 & 15.882 & 15.684 & 15.668 & 15.399 & 15.392 & 0.67 & 0.52 & 0.34 & $\ldots$ & $\ldots$ \\
\hline V132 & 10 & 0.33986 & 15.909 & 15.892 & 15.653 & 15.643 & 15.259 & 15.247 & 0.53 & 0.41 & 0.25 & $f_{0.61}$ & $\cdots$ \\
\hline V140 & 10 & 0.33316 & 15.743 & 15.722 & 15.515 & 15.502 & 15.155 & 15.150 & $0.69-0.60$ & $0.53-0.45$ & $0.32-0.27$ & $\ldots$ & $\mathrm{Bl}$ \\
\hline V147* & 10 & 0.34647 & $\cdots$ & $\cdots$ & $\cdots$ & $\cdots$ & $\ldots$ & $\cdots$ & $\ldots$ & $\ldots$ & $\ldots$ & $f_{0.61}$ & $\cdots$ \\
\hline V168 & 10 & 0.27594 & 15.803 & 15.788 & 15.588 & 15.580 & 15.345 & 15.342 & $0.65-0.46$ & $0.48-0.35$ & $0.32-0.23$ & $\ldots$ & B1 \\
\hline V170* & 10 & 0.43238 & $\ldots$ & $\ldots$ & $\ldots$ & $\ldots$ & $\ldots$ & $\ldots$ & $\cdots$ & $\ldots$ & $\cdots$ & $\cdots$ & $\ldots$ \\
\hline V171 & 10 & 0.30328 & $\ldots$ & $\ldots$ & $\ldots$ & $\ldots$ & $\ldots$ & $\ldots$ & $\cdots$ & $\cdots$ & $\ldots$ & $\ldots$ & $\ldots$ \\
\hline V177 & 10 & 0.34836 & 15.763 & 15.735 & 15.552 & 15.536 & 15.263 & 15.257 & 0.67 & 0.52 & 0.33 & $\ldots$ & $\ldots$ \\
\hline V188* & 2O: & 0.26651 & $\ldots$ & $\ldots$ & $\cdots$ & $\ldots$ & $\ldots$ & $\ldots$ & $\ldots$ & $\ldots$ & $\ldots$ & $\ldots$ & $\ldots$ \\
\hline V203 & $2 \mathrm{O}$ & 0.28979 & 15.766 & 15.764 & 15.581 & 15.580 & 15.296 & 15.296 & $0.21-0.17$ & $0.17-0.12$ & $0.09-0.08$ & $\cdots$ & $\mathrm{B} 1$ \\
\hline V207 & 10 & 0.34530 & $\ldots$ & $\ldots$ & $\ldots$ & $\ldots$ & $\ldots$ & $\ldots$ & $\ldots$ & $\ldots$ & $\ldots$ & $\ldots$ & $\ldots$ \\
\hline V208 & 10 & 0.33838 & 15.923 & 15.905 & 15.647 & 15.638 & 15.266 & 15.262 & 0.55 & 0.40 & 0.26 & $f_{0.61}$ & $\cdots$ \\
\hline V209 & 10 & 0.34829 & $\cdots$ & $\cdots$ & $\cdots$ & $\cdots$ & $\cdots$ & $\cdots$ & $\cdots$ & $\cdots$ & $\cdots$ & $f_{0.61}$ & $\cdots$ \\
\hline V210 & 10 & 0.35295 & $\cdots$ & $\cdots$ & $\cdots$ & $\cdots$ & $\ldots$ & $\cdots$ & $\cdots$ & $\cdots$ & $\cdots$ & $f_{0.61}$ & $\cdots$ \\
\hline V213* & 10 & 0.30001 & $\cdots$ & $\cdots$ & $\cdots$ & $\cdots$ & $\cdots$ & $\cdots$ & $\cdots$ & $\cdots$ & $\cdots$ & $\cdots$ & $\ldots$ \\
\hline V216 & 10 & 0.34648 & $\ldots$ & $\cdots$ & $\ldots$ & $\ldots$ & $\ldots$ & $\ldots$ & $\ldots$ & $\ldots$ & $\ldots$ & $\ldots$ & $\ldots$ \\
\hline V221 & 10 & 0.37878 & $\ldots$ & $\cdots$ & $\ldots$ & $\ldots$ & $\ldots$ & $\ldots$ & $\ldots$ & $\cdots$ & $\cdots$ & $\ldots$ & $\ldots$ \\
\hline V223 & 10 & 0.32921 & $\ldots$ & $\cdots$ & $\ldots$ & $\ldots$ & $\ldots$ & $\ldots$ & $\ldots$ & $\cdots$ & $\cdots$ & $\ldots$ & $\ldots$ \\
\hline V240 & 10 & 0.27601 & $\ldots$ & $\cdots$ & $\ldots$ & $\ldots$ & $\ldots$ & $\ldots$ & $\ldots$ & $\cdots$ & $\ldots$ & $\ldots$ & $\ldots$ \\
\hline $\mathrm{V} 245^{*}$ & 10 & 0.28403 & $\ldots$ & $\ldots$ & $\ldots$ & $\ldots$ & $\ldots$ & $\ldots$ & $\ldots$ & $\ldots$ & $\ldots$ & $\ldots$ & $\ldots$ \\
\hline V246* & 10 & 0.33916 & $\ldots$ & $\ldots$ & $\ldots$ & $\ldots$ & $\ldots$ & $\ldots$ & $\ldots$ & $\cdots$ & $\ldots$ & $\ldots$ & $\ldots$ \\
\hline V253* & 10 & 0.33260 & $\cdots$ & $\cdots$ & $\cdots$ & $\cdots$ & $\cdots$ & $\cdots$ & $\cdots$ & $\cdots$ & $\cdots$ & $f_{0.61}$ & $\cdots$ \\
\hline V256 & 10 & 0.31806 & $\cdots$ & $\cdots$ & $\cdots$ & $\cdots$ & $\cdots$ & $\cdots$ & $\cdots$ & $\cdots$ & $\cdots$ & $\cdots$ & $\cdots$ \\
\hline V259 & 10 & 0.33351 & $\cdots$ & $\cdots$ & $\cdots$ & $\cdots$ & $\cdots$ & $\cdots$ & $\cdots$ & $\cdots$ & $\cdots$ & $f_{0.61}$ & $\cdots$ \\
\hline V261* & 10 & 0.44473 & $\cdots$ & $\cdots$ & $\cdots$ & $\cdots$ & $\cdots$ & $\cdots$ & $\cdots$ & $\cdots$ & $\cdots$ & $f_{0.61}$ & $\cdots$ \\
\hline V264* & 10 & 0.35646 & $\cdots$ & $\cdots$ & $\cdots$ & $\cdots$ & $\ldots$ & $\cdots$ & $\cdots$ & $\cdots$ & $\cdots$ & $\cdots$ & $\ldots$ \\
\hline V290 & $2 \mathrm{O}$ & 0.24041 & 15.861 & 15.861 & 15.679 & 15.679 & 15.415 & 15.415 & 0.05 & 0.04 & 0.03 & $\cdots$ & $\cdots$ \\
\hline V292 & 2O: & 0.29654 & $\cdots$ & $\cdots$ & $\cdots$ & $\cdots$ & $\cdots$ & $\cdots$ & $\cdots$ & $\cdots$ & $\cdots$ & $\cdots$ & \\
\hline
\end{tabular}

Notes.

a Asterisk denotes stars with photometry contaminated by signals of nearby variables.

b 1O: first overtone; 2O: second overtone.

${ }^{c}\langle\rangle$ and () denote magnitude- and intensity-averaged mean magnitudes, respectively.

${ }^{\mathrm{d}}$ Peak to peak amplitudes. Maximum and minimum amplitudes are given for Blazhko satrs.

e $f_{0.61}$ : frequency at $f_{10} / f_{0.61} \approx 0.61-0.62 ; \mathrm{Bl}$ : Blazhko effect.

overtone (blue circle), and second-overtone (black square) stars are located in separated groups, with some overlapping in each plot. The extra properties of the stars, the Blazhko effect, and the $f_{0.61}$ additional frequency are also indicated in the figure.

The Fourier amplitudes, amplitude ratios, and phase differences of the $V$ light curves are shown in Figure 4. The same symbols are used for distinguishing stars with different properties as in Figure 3. The location of different-type variables in Figures 3 and 4 are discussed in the corresponding sections.

\subsection{Multi-mode Pulsations, Additional Frequencies}

The light curves of more than half of the studied stars are not stable; their Fourier spectra show extra frequency components. 
Table 4

Summary of the Photometric Properties of Double/Triple-mode Variables in M3

\begin{tabular}{|c|c|c|c|c|c|c|c|c|c|c|c|c|c|c|c|}
\hline $\operatorname{Star}^{\mathrm{a}}$ & Mode $^{\mathrm{b}}$ & $\begin{array}{l}\text { Period } \\
\text { (days) }\end{array}$ & $\begin{array}{c}\langle B\rangle^{\mathrm{c}} \\
(\mathrm{mag})\end{array}$ & $\begin{array}{c}(B)^{\mathrm{c}} \\
(\mathrm{mag})\end{array}$ & $\begin{array}{c}\langle V\rangle \\
(\mathrm{mag})\end{array}$ & $\begin{array}{c}(V) \\
(\mathrm{mag})\end{array}$ & $\begin{array}{c}\left\langle I_{\mathrm{C}}\right\rangle \\
(\mathrm{mag})\end{array}$ & $\begin{array}{c}\left(I_{\mathrm{C}}\right) \\
(\mathrm{mag})\end{array}$ & $\begin{array}{c}A_{B}^{\mathrm{d}} \\
(\mathrm{mag})\end{array}$ & $\begin{array}{c}A_{V}^{\mathrm{d}} \\
(\mathrm{mag})\end{array}$ & $\begin{array}{c}A_{I_{\mathrm{C}}}^{\mathrm{d}} \\
(\mathrm{mag})\end{array}$ & $R^{\mathrm{e}}$ & \multicolumn{3}{|c|}{$\begin{array}{l}\text { Additional } \\
\text { Feature }^{f}\end{array}$} \\
\hline \multirow[t]{2}{*}{$\mathrm{V} 13$} & 10 & 0.35072 & 15.986 & 15.953 & 15.690 & 15.673 & 15.279 & 15.271 & $0.38-0.11$ & $0.29-0.09$ & $0.19-0.05$ & $\ldots$ & $f_{0.61}$ & $\mathrm{~B} 1$ & $\cdots$ \\
\hline & $2 \mathrm{O}$ & 0.28160 & $\ldots$ & $\ldots$ & $\ldots$ & $\ldots$ & $\ldots$ & $\ldots$ & 0.020 & 0.019 & 0.010 & $\ldots$ & $\ldots$ & $\ldots$ & PD \\
\hline \multirow[t]{2}{*}{ V44 } & 10 & 0.36812 & 15.951 & 15.928 & 15.659 & 15.646 & 15.220 & 15.215 & $0.25-0.14$ & $0.19-0.11$ & $0.12-0.07$ & $\ldots$ & $\ldots$ & $\mathrm{B} 1$ & $\ldots$ \\
\hline & F & 0.50377 & $\ldots$ & $\ldots$ & $\ldots$ & $\ldots$ & $\ldots$ & $\cdots$ & $0.87-0.34$ & $0.62-0.24$ & $0.40-0.19$ & $\ldots$ & $\ldots$ & $\mathrm{B} 1$ & $\ldots$ \\
\hline \multirow[t]{2}{*}{ V87 } & 10 & 0.35749 & 15.871 & 15.853 & 15.583 & 15.573 & 15.168 & 15.164 & 0.49 & 0.37 & 0.23 & $\cdots$ & $f_{0.61}$ & $\cdots$ & $\cdots$ \\
\hline & F & 0.48017 & $\ldots$ & $\ldots$ & $\ldots$ & $\ldots$ & $\ldots$ & $\ldots$ & 0.21 & 0.16 & 0.11 & 0.4 & $\ldots$ & $\ldots$ & $\ldots$ \\
\hline \multirow[t]{2}{*}{ V99 } & 10 & 0.3611 & 15.940 & 15.924 & 15.653 & 15.644 & 15.223 & 15.220 & $0.52-0.13$ & $0.39-0.10$ & $0.26-0.06$ & $\ldots$ & $\cdots$ & $\mathrm{Bl}$ & \\
\hline & $\mathrm{F}$ & 0.4821 & $\ldots$ & $\ldots$ & $\ldots$ & $\ldots$ & $\ldots$ & $\ldots$ & $0.44-0.20$ & $0.38-0.15$ & $0.24-0.10$ & $\ldots$ & $\ldots$ & $\mathrm{B} 1$ & ... \\
\hline \multirow[t]{2}{*}{ V125 } & 10 & 0.34982 & 15.939 & 15.922 & 15.665 & 15.656 & 15.262 & 15.258 & 0.54 & 0.42 & 0.26 & 0.02 & $f_{0.61}$ & $\cdots$ & $\ldots$ \\
\hline & F & 0.4709 & $\ldots$ & $\ldots$ & $\ldots$ & $\ldots$ & $\ldots$ & $\ldots$ & 0.012 & 0.012 & 0.005 & $\ldots$ & $\ldots$ & $\ldots$ & \\
\hline \multirow[t]{2}{*}{ V251* } & 10 & 0.3542 & $\ldots$ & $\ldots$ & $\ldots$ & $\ldots$ & $\ldots$ & $\ldots$ & $\ldots$ & $\ldots$ & $\ldots$ & $\ldots$ & $\ldots$ & $\ldots$ & ... \\
\hline & $\mathrm{F}$ & 0.4762 & $\ldots$ & $\ldots$ & $\ldots$ & $\ldots$ & $\ldots$ & $\ldots$ & $\ldots$ & $\ldots$ & $\ldots$ & 0.9 & $\ldots$ & $\ldots$ & ... \\
\hline \multirow[t]{2}{*}{$\mathrm{V} 252^{*}$} & 10 & 0.3361 & $\ldots$ & $\ldots$ & $\ldots$ & $\ldots$ & $\ldots$ & $\ldots$ & $\ldots$ & $\ldots$ & $\ldots$ & $\ldots$ & $\ldots$ & $\ldots$ & $\cdots$ \\
\hline & $\mathrm{F}$ & 0.4530 & $\ldots$ & $\ldots$ & $\ldots$ & $\ldots$ & $\ldots$ & $\ldots$ & $\ldots$ & $\ldots$ & $\ldots$ & 0.95 & $\ldots$ & $\ldots$ & .. \\
\hline
\end{tabular}

Notes.

a Asterisk denotes stars with photometry contaminated by signals of nearby variables.

${ }^{\mathrm{b}} \mathrm{F}$ : fundamental mode; 10: first overtone; 2O: second overtone.

${ }^{c}\langle\rangle$ and () denote magnitude- and intensity-averaged mean magnitudes, respectively.

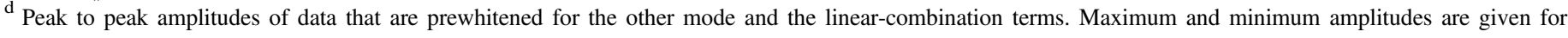
Blazhko stars.

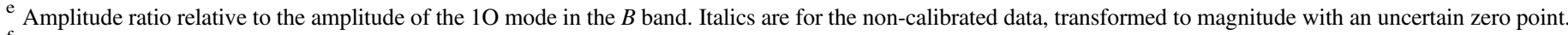

f $f_{0.61}$ : frequency at $f_{10} / f_{0.61} \approx 0.61-0.62 ; \mathrm{Bl}$ : Blazhko effect; PD: period doubling.

$\mathrm{g}$ The $f_{0.61}$ frequency is detected only in the $V$ data.

For example, an additional frequency component appears in 18 stars at the frequency ratio $f_{10} / f_{0.61} \approx 0.61-0.62\left(\mathrm{RR}_{0.61}\right.$ stars). PD-manifested as a signal at a half-integer frequency ratio to the original one-is detected in two cases (V13 and V166). When the additional frequency appears close to the overtone frequency (and its harmonics), the Blazhko effect (B1) is supposed to be observed, even if only one close frequency component is detected (V126, V166, and V203). Alternatively, however, the additional signal may correspond to a non-radial mode in these latter cases.

Ten double-mode variables are in the sample; one of them is, in fact, a triple-mode star. Aside from the fundamental $(\mathrm{F})$ and the first-overtone $(1 \mathrm{O})$ modes, the second-overtone mode $(2 \mathrm{O}$; the identification is based on the $P / P_{\mathrm{F}}=0.587$ period ratio) also appears in the spectrum of V13. If the $2 \mathrm{O}$ identification of the third mode is correct, V13 is the first RR Lyrae star to show pulsation with the first three radial modes simultaneously.

The occurrence of the different types of phenomena are frequently interlaced. The most complex variation is observed in the triple-mode star, V13, which shows the Blazhko effect of the $\mathrm{F}$ and $1 \mathrm{O}$ radial modes, $\mathrm{PD}$ of the $2 \mathrm{O}$ mode, and the $f_{0.61}$ component. About half of the RRd stars show the Blazhko effect and their $P_{\mathrm{O}} / P_{\mathrm{F}}$ period ratios are anomalous.

In contrast with the fast and irregular period changes that are often detected in RRc stars, the period changes of the overtone variables in M3 are marginal or modest. Irregular, rapid period changes were detected only in two RRc stars (V12 and V70) and some RRd stars in the period-change study of the RR Lyrae stars of M3 (J12). Therefore, the possibility that the detected additional features were the artifacts of period changes in a 200 day long time-series photometry can be excluded in most cases. The possible role of the period changes of V70 is discussed in Section 6.1, and the period changes of doublemode stars are summarized in Section 7.

\subsection{Evolved and Second-overtone Variables}

The overtone variables (V70, V170, and V261) with the longest periods are close to the short-end of the period range of the fundamental-mode variables $(P=0.40-0.45$ days $)$ and have light curves showing anomalous shape. Their rising branch is longer than that of typical ones, and the bump appears at its middle, while that generally precedes the maximum light in normal RRc stars slightly. Therefore, the RRc status of these highly evolved variables might be questionable. However, two of these stars (V70 and V261) show a secondary periodicity with 0.61 period ratio. The fact that this type of multiperiodicity appears in many other RRc stars in M3, and exclusively in first-overtone variables according to other studies (see details in Section 4), supports the RRc status of these stars.

The identification of three $2 \mathrm{O}$ variables (V105, V203, and V290) is based on their short period, small-amplitude, smooth light curves and extreme color indices. Although its period and 

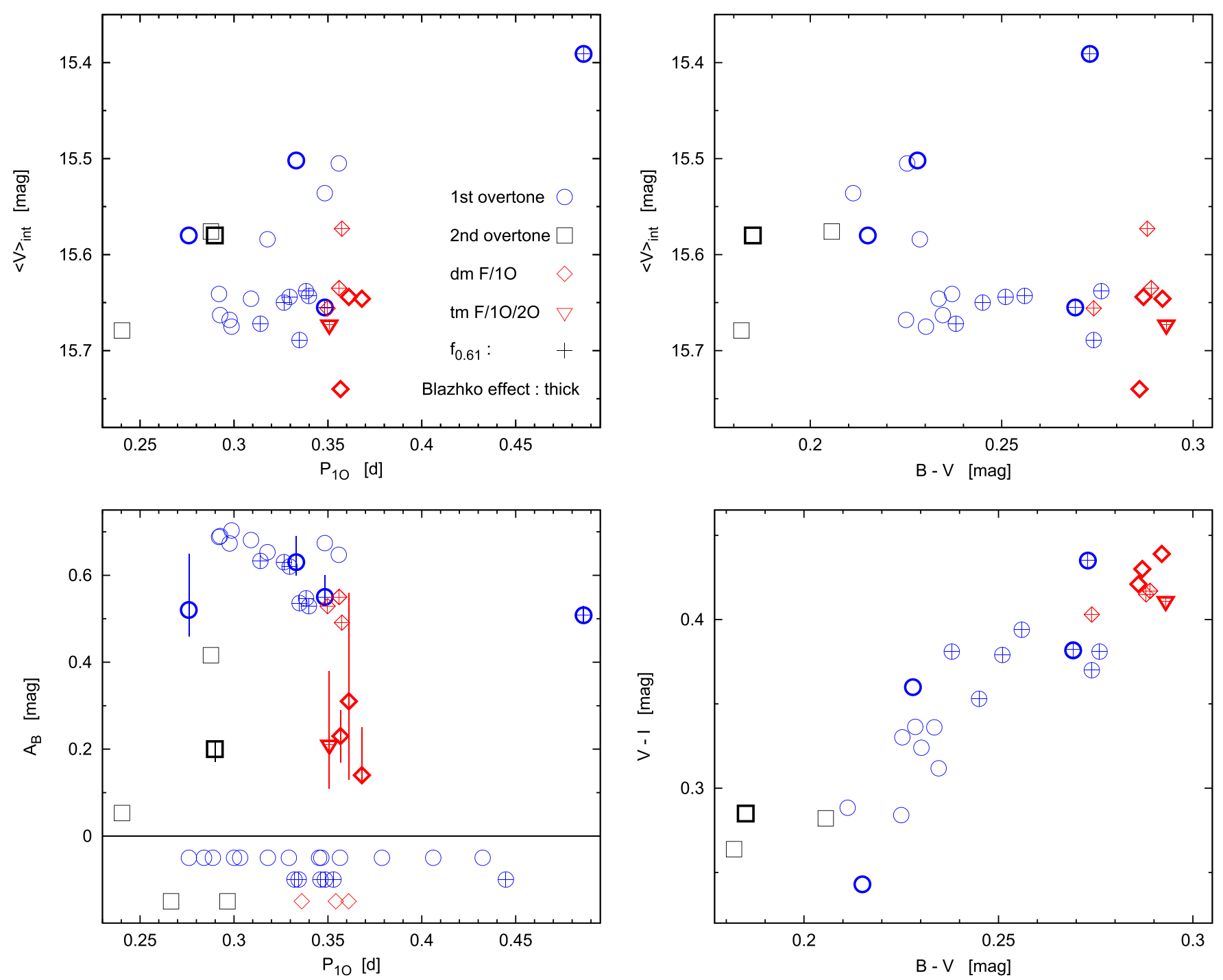

Figure 3. Intensity-averaged mean $V$ magnitudes and peak-to-peak $B$ amplitudes vs. period of overtone variables are plotted on the left-side panels. $1 \mathrm{O}, 2 \mathrm{O}$ variables, $\mathrm{F} / 1 \mathrm{O}$ stars, and the $\mathrm{F} / 1 \mathrm{O} / 2 \mathrm{O}$ triple-mode variable, V13, are denoted by different symbols. The appearance of the Blazhko effect and the additional frequency component at the $f_{10} / f_{0.61} \approx 0.61-0.62$ ratio is also indicated. The period distribution of stars with an uncertain flux-magnitude transformation is shown in the bottom part of the $A(B)-P_{10}$ panel. For Blazhko stars, the full range of the amplitude variation is indicated by a vertical line. The right-hand panels show the intensityaveraged mean $V$ magnitude (top) and $V-I$ color (bottom) vs. $B-V$.

amplitude may also indicate $2 \mathrm{O}$ status of V168 $(P=0.276$ days), this star shows a strong bump preceding maximum light in the large-amplitude phase of its modulation, which is a feature typical in RRc stars, thus its $2 \mathrm{O}$ classification is rejected. The second-overtone status of two more stars with no color information (V188 and V292) is also proposed based on their short periods and small-amplitude smooth light curves.

\section{ADDITIONAL FREQUENCIES AT THE 0.61 FREQUENCY RATIO}

The $f_{0.61}$ frequency $\left(f_{0.61} \approx f_{1 \mathrm{O}} / 0.61\right)$ is detected in $14 \mathrm{RRc}$ and in four double-mode stars in M3. Thus, the phenomenon is observed in $38 \%$ and $40 \%$ of RRc and RRd stars, respectively. Figures 5 and 6 show the spectral windows, the amplitude spectra, and the prewhitened spectra of V75 and V97, as examples for the detection of the $f_{0.61}$ frequency in the D12 data.

Table 5 lists the $\mathrm{RR}_{0.61}$ stars and gives their frequencies, frequency ratios, $A_{0.61}$ amplitudes in $V$ band, and the $A_{0.61} / A_{1 O}$ amplitude ratios. The previous CCD data (C01, K98, H05, $\mathrm{B} 06$, and J12) of these variables have also been reinvestigated and the $f_{0.61}$ frequency was detected in six stars in the archive photometric data; these results are also given in Table 5.

\subsection{Stability of the $f_{0.61}$ Frequency}

The $f_{0.61}$ frequencies detected in the archive CCD and D12 data differ from each other in most of the cases and the differences are too large to be explained by the $\sim 0.0001$ -0.001 day $^{-1}$ uncertainty of the frequencies.

The $f_{0.61}$ frequency is a discrete, stable signal in V75, but this component has a complex, multiple structure in V97, which indicates probable amplitude and/or phase changes of the signal. A complex structure of the $f_{0.61}$ frequency is detected in the residual spectra of four variables in the D12 data. The stability of the frequency solution of these stars is checked on V97. The first and second parts of the observations are analyzed separately, and these results are also given in Table 5 . The frequency and the amplitude of the $f_{0.61}$ component are 

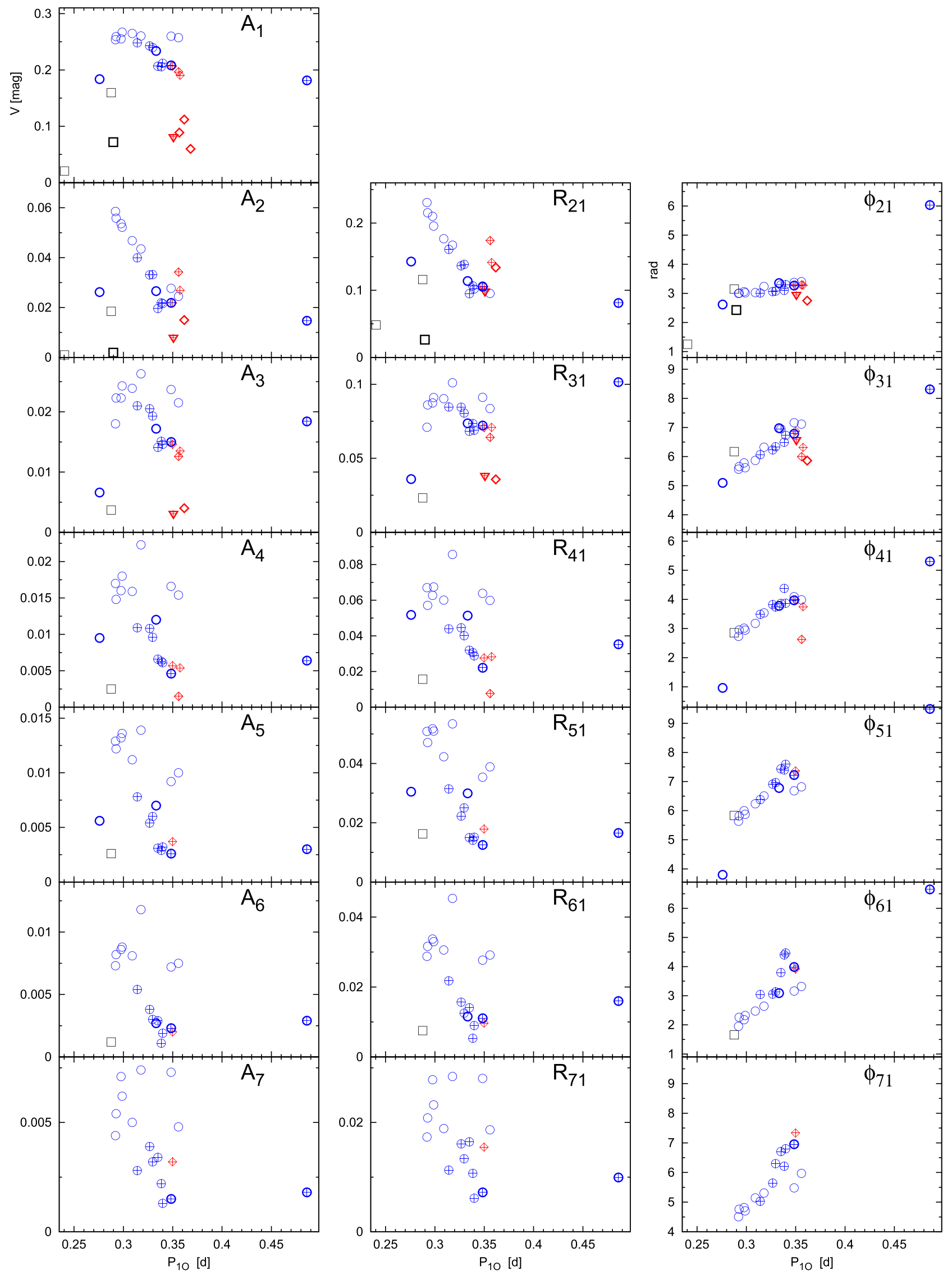

Figure 4. Fourier amplitudes $\left(A_{k}\right)$, amplitude ratios $\left(R_{k 1}\right)$, and phase differences $\left(\varphi_{k 1}\right)$ of the $V$ light curves of overtone variables and the overtone mode of doublemode stars are shown. The symbols are the same as in Figure 3. Low-amplitude variables (second overtone, double-mode stars) do not appear in each plot because they have detectable amplitudes only in the lowest harmonic orders. 


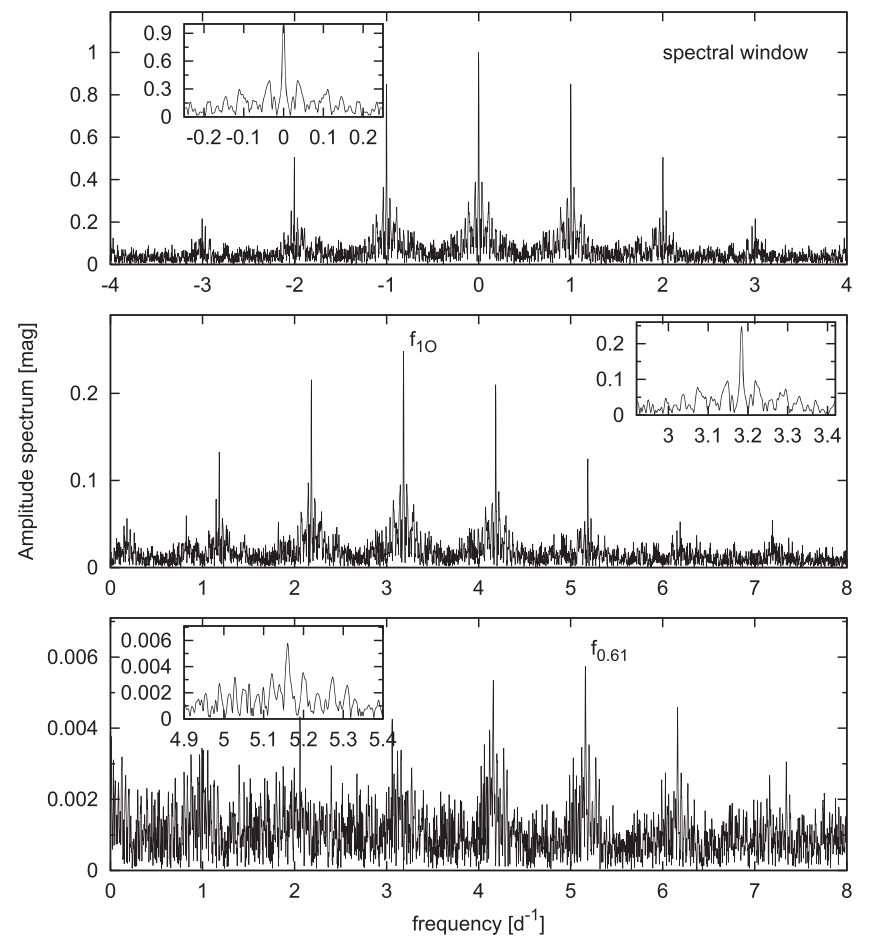

Figure 5. Spectral window (top), amplitude spectrum, and spectrum of the data prewhitened for the overtone-mode frequencies of V75 in the $V$ band. The insets enlarge the main peaks. The detection of the $f_{0.61}$ frequency in the prewitened spectrum of V13 is shown in Section 5.
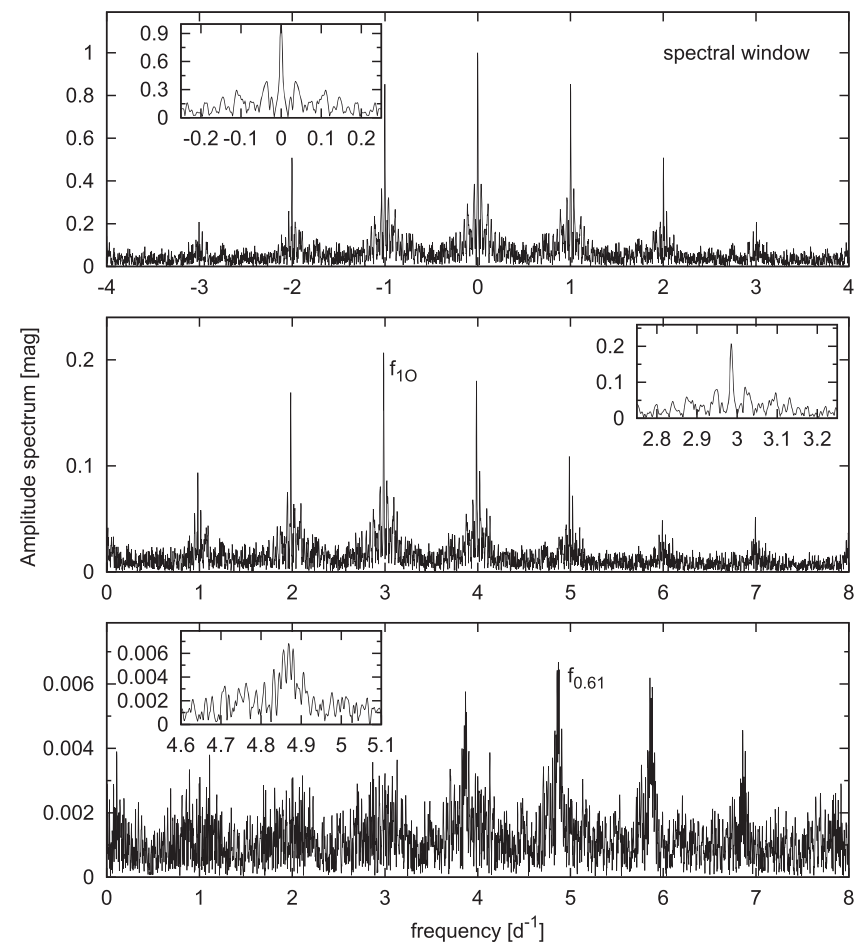

Figure 6. The same spectra as in Figure 5 for V97. The $f_{0.61}$ frequency is a complex multiplet in this case.

significantly different in the two parts of the data. This, and the differences between the results of the D12 and the archive data imply that, instead of real multiplets, the amplitude and/or frequency changes of the $f_{0.61}$ component should be behind its
Table 5

Parameters of the $f_{0.61}$ Frequency Components

\begin{tabular}{|c|c|c|c|c|c|c|}
\hline Star & $\begin{array}{c}f_{1 \mathrm{O}} \\
\left(\mathrm{day}^{-1}\right)\end{array}$ & $\begin{array}{c}f_{0.61} \\
\left(\text { day }^{-1}\right)\end{array}$ & $f_{10} / f_{0.61}$ & $\begin{array}{l}A_{0.61}{ }^{\mathrm{a}} \\
(\mathrm{mag})\end{array}$ & $A_{0.61} / A_{10}{ }^{\mathrm{b}}$ & Data $^{c}$ \\
\hline V13 & 2.8513 & 4.6457 & 0.6137 & 0.006 & 0.075 & D12 \\
\hline \multirow[t]{3}{*}{ V37 } & 3.0615 & 4.977 & 0.6151 & 0.006 & 0.025 & D12 \\
\hline & $\ldots$ & 4.965 & 0.6166 & 0.004 & 0.018 & D12 \\
\hline & 3.0615 & 4.9660 & 0.6165 & 0.007 & 0.028 & $\mathrm{H} 05+\mathrm{B} 06$ \\
\hline \multirow[t]{4}{*}{ V56 } & 3.0340 & 4.944 & 0.6137 & 0.009 & 0.036 & D12 \\
\hline & $\ldots$ & 4.911 & 0.6137 & 0.005 & 0.019 & D12 \\
\hline & 3.0341 & 4.9475 & 0.6133 & 0.007 & 0.028 & $\mathrm{~J} 12$ \\
\hline & 3.0339 & 4.9323 & 0.6151 & 0.009 & 0.035 & $\mathrm{H} 05+\mathrm{B} 06$ \\
\hline V68 & 2.8092 & 4.5717 & 0.6145 & 0.005 & 0.028 & D12 \\
\hline V70 & 2.0573 & 3.3115: & 0.6213 & 0.004 & 0.024 & D12 \\
\hline \multirow[t]{2}{*}{ V75 } & 3.1839 & 5.1595 & 0.6171 & 0.006 & 0.023 & D12 \\
\hline & 3.1840 & 5.1506 & 0.6182 & 0.008 & 0.032 & $\mathrm{~J} 12$ \\
\hline V87 & 2.7973 & 4.5288 & 0.6177 & 0.006 & 0.034 & D12 \\
\hline \multirow[t]{6}{*}{ V97 } & 2.9857 & 4.879 & 0.6147 & 0.005 & 0.026 & D12 \\
\hline & $\cdots$ & 4.868 & 0.6133 & 0.005 & 0.025 & D12 \\
\hline & $\cdots$ & 4.857 & 0.6119 & 0.005 & 0.024 & D12 \\
\hline & 2.9856 & 4.8597 & 0.6144 & 0.010 & 0.050 & D12a \\
\hline & 2.9857 & 4.8703 & 0.6130 & 0.005 & 0.022 & D12b \\
\hline & 2.9857 & 4.8370 & 0.6173 & 0.008 & 0.041 & $\mathrm{H} 05+\mathrm{B} 06$ \\
\hline \multirow[t]{5}{*}{ V125 } & 2.8586 & 4.654 & 0.6142 & 0.006 & 0.028 & D12 \\
\hline & $\cdots$ & 4.640 & 0.6161 & 0.005 & 0.022 & D12 \\
\hline & $\cdots$ & 4.590 & 0.6229 & 0.005 & 0.025 & D12 \\
\hline & 2.8586 & 4.6343 & 0.6168 & 0.006 & 0.031 & $\mathrm{~J} 12$ \\
\hline & 2.8587 & 4.6344 & 0.6168 & 0.009 & 0.047 & $\mathrm{H} 05+\mathrm{B} 06$ \\
\hline V126 & 2.8702 & 4.604: & 0.6234 & 0.005 & 0.023 & D12 \\
\hline \multirow[t]{2}{*}{ V132 } & 2.9424 & 4.7913 & 0.6141 & 0.011 & 0.053 & D12 \\
\hline & 2.9425 & 4.7497 & 0.6195 & 0.011 & 0.057 & B06 \\
\hline V147 & 2.8863 & 4.6947 & 0.6148 & $\ldots$ & 0.028 & D12 \\
\hline V208 & 2.9552 & 4.8058 & 0.6149 & 0.013 & 0.065 & D12 \\
\hline V209 & 2.8712 & 4.6800 & 0.6135 & $\ldots$ & 0.033 & D12 \\
\hline V210 & 2.8333 & 4.6185 & 0.6135 & $\cdots$ & 0.044 & D12 \\
\hline V253 & 3.0066 & 4.8720 & 0.6171 & $\cdots$ & 0.079 & D12 \\
\hline V259 & 2.9984 & 4.8773 & 0.6148 & $\cdots$ & 0.026 & D12 \\
\hline V261 & 2.2485 & 3.6237 & 0.6205 & $\ldots$ & 0.069 & D12 \\
\hline
\end{tabular}

Notes.

${ }^{\text {a }}$ Fourier amplitude in the $V$ band.

${ }^{\mathrm{b}}$ Amplitude ratio in the $V$ band. Italics denote the $B$-band amplitude ratio of non-calibrated data.

${ }^{\mathrm{c}}$ D12a and D12b denote the first and second parts of the D12 data.

complex appearance. This is in contrast with the stability of the radial-mode frequency of the $\mathrm{RR}_{0.61}$ stars. While as large as $\sim 1 \%$ differences are detected in the frequency values of the $f_{0.61}$ component, the variation in the frequency of the radial mode is lower than $0.01 \%$ (see Table 5).

\subsection{On the Light Curves of $R R_{0.61}$ Stars}

The appearance of the $f_{0.61}$ frequency in different groups of stars is indicated in Figures 1 and 2. The light curves of these variables are more sinusoidal than the light curves of overtone variables without any additional features. While a pronounced bump characterizes the light variation of normal RRc stars just preceding light maximum, the bump in these stars is marginal if it exists at all. The light curves of the 10 mode of double-mode stars have a similar shape as $\mathrm{RR}_{0.61}$ stars, irrespective of whether or not the $f_{0.61}$ frequency is detected in their spectra (see also Figure 20 in Section 7).

According to the best-quality light curves shown in Figure 1, there is no star contradicting this scheme. Each $R_{0.61}$ star 

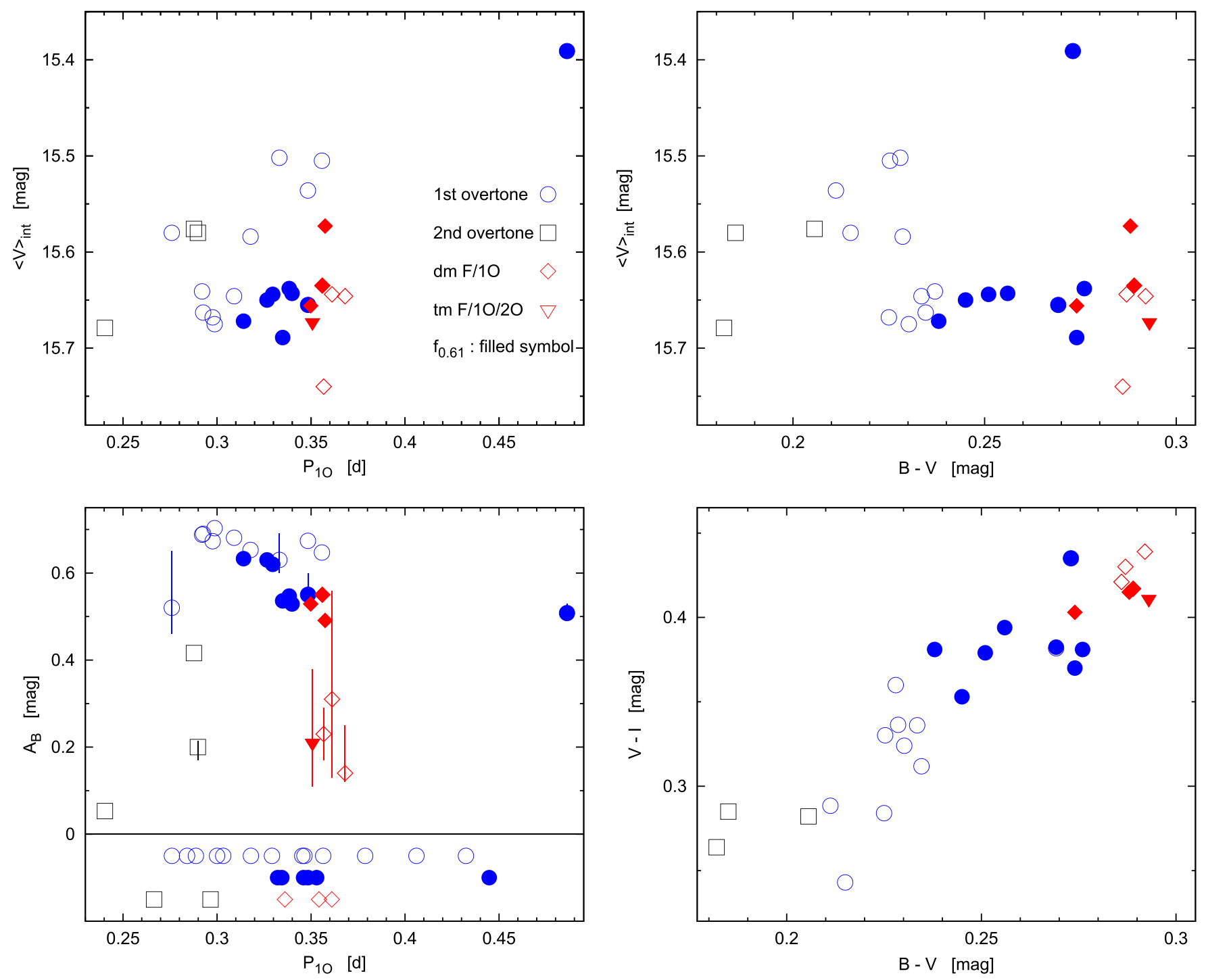

Figure 7. The same as in Figure $3 . \mathrm{RR}_{0.61}$ stars are shown by filled symbols.

displays a sinusoidal light curve with a strongly reduced bump, similar to the overtone-mode light curve of double-mode stars, whereas the light curves of the normal RRc stars are asymmetric and their bump is pronounced. The only exception is V70, which is a highly evolved, over-luminous, long-period RRc star.

The non-calibrated light curves of variables affected by crowding shown in Figure 2 follow the same pattern with some exceptions. Each $\mathrm{RR}_{0.61}$ star of this sample fits to this scheme; their bumps are marginal. However, similar, sinusoidal light curves are also found among normal RRc stars. Because of the larger noise level, the detection limit of the additional frequency is larger in these cases than in the best-quality light curves. Therefore, it cannot be ruled out that these "outliers" may, in fact, exhibit the " 0.61 " feature as well, but the quality of the light curve simply does not allow its detection. There is also an evolved, long-period star in this sample (V170), which shows the $f_{0.61}$ frequency.

The Fourier parameters of the light curves (Figure 4) also reflect the described diversity. While mono-periodic RRc stars have detectable amplitudes up to 10 or even larger harmonic orders in the $V$ band, we can find harmonics only up to 6-8 with amplitudes above the noise limit in stars showing the $f_{0.61}$ frequency. The Fourier amplitudes and amplitude ratios of the $\mathrm{RR}_{0.61}$ stars are systematically smaller than the ones of normal RRc stars. Only double-mode, Blazhko, and 20 stars have amplitudes and amplitude ratios as low as $\mathrm{RR}_{0.61}$ stars over the third harmonic order. The phase differences of the $\mathrm{RR}_{0.61}$ stars do not show any peculiar behavior, they follow the trend defined by other RRc stars.

\subsection{Parameters of $R R_{0.61}$ Stars}

The location of $\mathrm{RR}_{0.61}$ stars is also special in the panels shown in Figure 7. These plots are the same as in Figure 3, but the $\mathrm{RR}_{0.61}$ stars are marked by filled symbols for clarity. The $f_{0.61}$ frequency component is not detected in any of the stars with a period shorter than 0.31 days, whereas it appears in the residual spectrum of each, not over-luminous RRc stars at longer periods, and in four double-mode stars. None of normalperiod, bright, evolved RRc stars show the $f_{0.61}$ frequency, but it is detected in two of the five highly evolved stars. The periods of these $1 \mathrm{O}$ pulsators (V70, $P=0.486$ days and V261, $P=0.445$ days) are longer than the 10 period of double-mode 


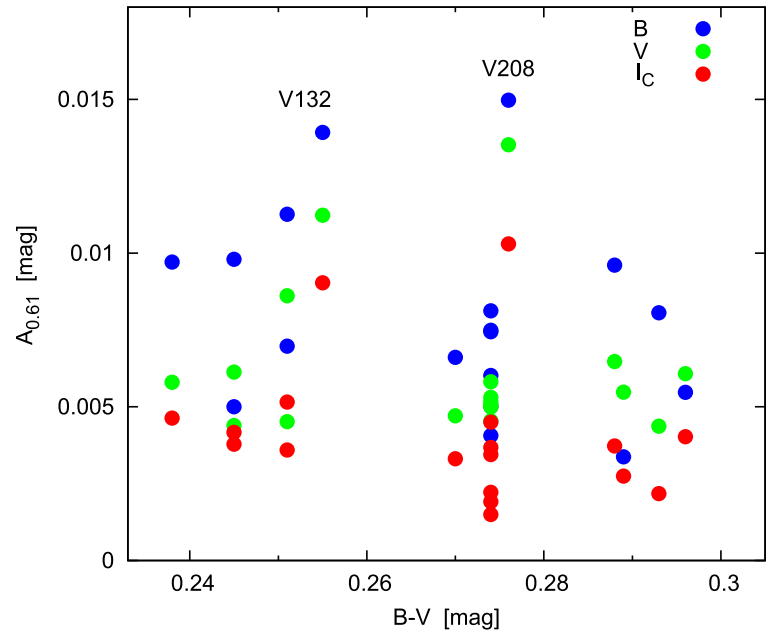

Figure 8. $B-, V-, I_{\mathrm{C}}$-band amplitudes of the $f_{0.61}$ frequency component vs. $B-V$. No amplitude decrease of the $f_{0.61}$ frequency toward smaller color indices is detected. The two largest $f_{0.61}$-amplitude stars are labeled.

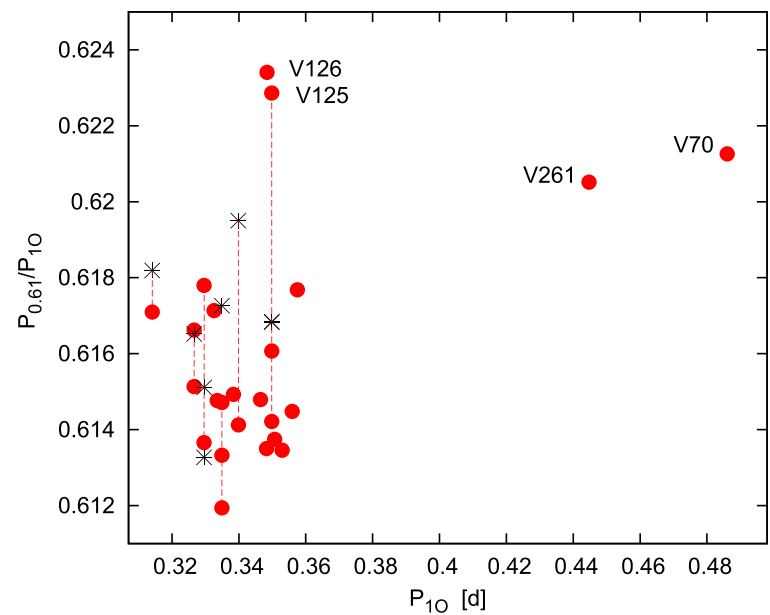

Figure 9. Petersen diagram, period ratio vs. $P_{10}$ of variables showing the $f_{0.61}$ frequency component. The detected frequencies of the same star are connected with dashed lines (see the data in Table 5). Results for archive CCD data are shown by asterisk.

stars; they are in the period range of the shortest period fundamental-mode variables. Although the magnitude calibration of V261 is uncertain-its period and light-curve shape is similar to those of V70, the brightest, longest-period RRc star in M3-it is very probable that there are no significant differences between the luminosities and temperatures of V261 and V70.

The separation of $\mathrm{RRc}$ and $\mathrm{RR}_{0.61}$ stars is the most pronounced in the $B-V-V-I$ panel of Figure 7. It seems that the $f_{0.61}$ phenomenon occurs only in the hotter half of the temperature range of overtone and double-mode stars in M3. There are only three double-mode stars (V44, V99, and V166) in the hotter half of the complete RRc/RRd sample where the $f_{0.61}$ frequency is not detected. However, these stars exhibit complex Blazhko modulation of both modes and the mean amplitude of their 10 mode is only about half of the amplitude of the other RRc stars and of RRd stars with dominant overtone mode. Supposing that the $A_{10} / A_{0.61}$ amplitude ratio is not extremely large (as in the case of V13, the only RRd star with dominant fundamental mode showing the $f_{0.61}$ component), the

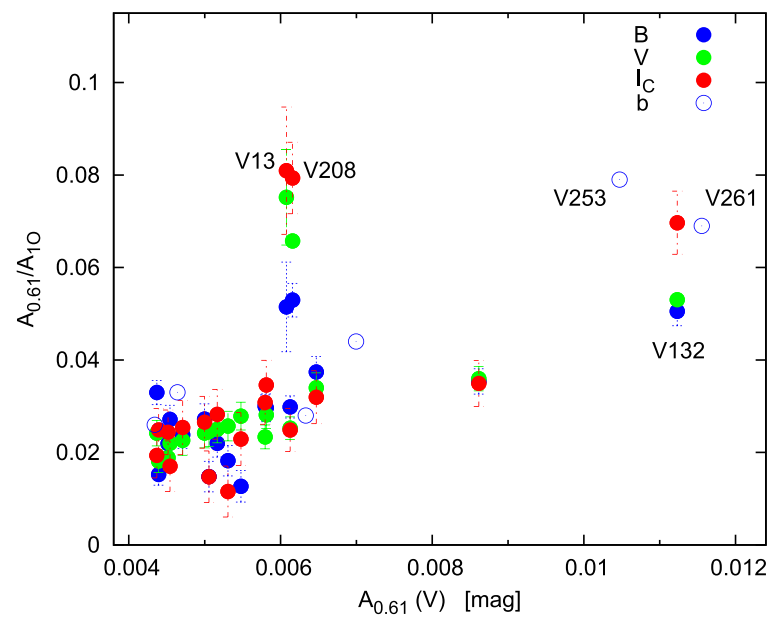

Figure 10. $B-, V$-, $I_{\mathrm{C}}$-band and non-calibrated $B$-filter amplitude ratios $\left(A_{0.61} / A_{10}\right)$ vs. the Fourier $V$ amplitude of the $f_{0.61}$ frequency ( $B$-filter amplitude of the non-calibrated data). For multiple $f_{0.61}$ signals each component is shown.

estimated amplitude of the $f_{0.61}$ frequency might be below the detection limit in these three RRd stars. The $f_{0.61}$ frequency is not detected in the RRd stars with uncalibrated, noisy light curves either, but the $f_{0.61}$ frequency may also be hidden in the noise in these stars. Therefore, we cannot exclude the possibility that the $f_{0.61}$ frequency is a common property of RRd stars and of the hotter half of the RRc sample. In contrast, none of the RRc stars with $B-V \lesssim 0.24$ and $V-I \lesssim 0.35$ mag shows the $f_{0.61}$ signal.

Figure 8 shows the amplitudes of the $f_{0.61}$ frequency components plotted against the $B-V$ color index. No systematic amplitude decrease of the $f_{0.61}$ frequency is detected with decreasing color index. Consequently, the lack of $\mathrm{RR}_{0.61}$ stars at hotter temperatures as shown in the $V-I$ versus $B-V$ panel of Figure 7 cannot be explained by a systematic reduction of the amplitude of the $f_{0.61}$ component toward hotter temperatures.

Figure 9 plots the observed $P_{0.61} / P_{10}$ period ratios of the 18 $\mathrm{RR}_{0.61}$ stars. Each component of the stars with multiple $f_{0.61}$ frequencies and results for the archive CCD data are shown. The period ratios fall in a very narrow range between 0.612 and 0.623 . The four largest values are detected in peculiar stars. Two of them, V70 and V261, are evolved, extremely longperiod variables. The additional frequency is a strongly varying signal with three $f_{0.61}$ components appearing in the whole data set of V125. The period ratios of two of these components and those obtained from the $\mathrm{H} 05, \mathrm{~B} 06$, and $\mathrm{J} 12$ data fall in the $0.614-0.617$ range. The largest period ratio is detected in V126. The $f_{0.61}$ component is one of the weakest in this star, thus in this case the frequency solution is somewhat uncertain.

Figure 10 shows the $A_{0.61} / A_{10}$ amplitude ratios in $B, V$, and $I_{\mathrm{C}}$ bands (and for the non-calibrated $B$-filter data) derived from the D12 data. Most of the amplitude ratios are in the 0.02-0.04 range. The amplitude ratios of five stars (V13, V132, V208, V253, and V261) are even larger; they are about $0.05-0.08$. The increased $A_{0.61} / A_{10}$ amplitude ratios are the consequences of either the relatively large amplitude of the $f_{0.61}$ component or the reduced amplitude of the overtone mode as in the case of V13. No general, systematic difference between the amplitude ratios in the $B, V, I_{\mathrm{C}}$ bands is seen for most of the $f_{0.61}$ components. However, the three large amplitude-ratio stars 

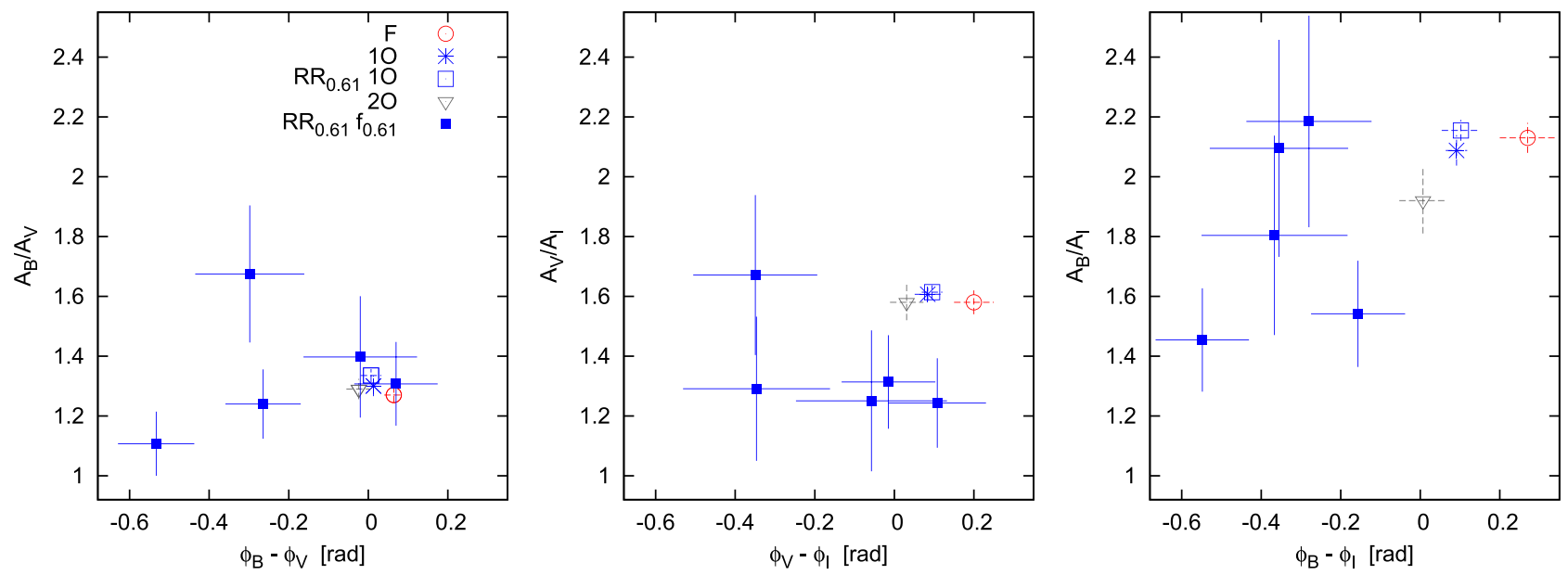

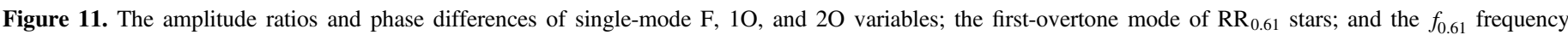

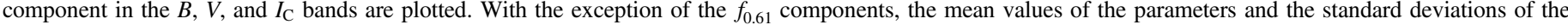

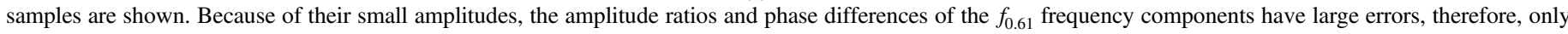
results of the five largest $\mathrm{S} / \mathrm{N}$ components are shown. The formal errors, which may underestimate the true uncertainties of the $f_{0.61}$ components, are indicated.

with $B V I$ photometric data (V13, V132, and V208) show an anomalous tendency-their $A_{0.61} / A_{10}$ amplitude ratio is the largest in the $I_{\mathrm{C}}$ and the smallest in the $B$ band. However, taking into account that the formal errors may seriously underestimate the true uncertainties, this result has to be taken with caution.

The amplitude ratios and phase differences of single-mode $\mathrm{F}$, $1 \mathrm{O}, 2 \mathrm{O}$ variables; the first-overtone mode of $\mathrm{RR}_{0.61}$ stars (mean values and standard deviations of the samples); and the $f_{0.61}$ frequency component are plotted in Figure 11 for the $B, V$, and $I_{\mathrm{C}}$ bands. The amplitude ratios and phase differences of the $f_{0.61}$ components have large errors because of their small amplitudes. Therefore, results are shown only for the five best, smallest-error components. There is no detectable difference between the positions of the overtone mode of the monoperiodic and the $\mathrm{RR}_{0.61}$ stars shown by an asterisk and a square, respectively. The phase differences of the $\mathrm{F}$ and $2 \mathrm{O}$ modes are at slightly larger and smaller values than the phase differences of the 1O. The amplitude ratios and phase differences of the best $\mathrm{S} / \mathrm{N} f_{0.61}$ components form a separate group in the $V, I$ and $B$, I planes, with no overlap with the regions of the radial modes. Although the errors even of the best signals are significant, the homogeneous location of these components implies that the amplitude ratios and phase differences of the $f_{0.61}$ frequency and of the radial modes may indeed be different. However, more accurate multicolor observations of $\mathrm{RR}_{0.61}$ stars are needed to locate the precise positions of the $f_{0.61}$ component in the amplitude-ratio and phase-difference planes.

\subsection{Comparison with Other Results}

The $f_{0.61}$ frequency has been detected in several overtone RR Lyrae stars previously. A complete list of these objects was given by Moskalik et al. (2015). Moreover, Netzel et al. (2015) identified 147 Galactic bulge objects in the Optical Gravitational Lensing Experiment (OGLE)-III photometry that all showed the peculiar frequency component in the 0.60-0.65 period-ratio range. The period ratios of the OGLE sample defined two sequences, one at 0.613 and a less populous group at 0.631 ratio. Moskalik et al. (2015) argued that this frequency cannot be identified with any radial mode. They have concluded that the $f_{0.61}$ frequency most probably corresponds to a strongly trapped, unstable, high-degree $(l \geqslant 6)$ nonradial mode.

Color information may help mode identification of nonradial modes (see, e.g., Garrido 2004), however, RR Lyrae stars still lack appropriate modeling to exploit this possibility.

Similar period ratios were detected in numerous Magellanic Cloud 10 Cepheids (Moskalik \& Kołaczkowski 2008, 2009; Soszyński et al. 2008, 2010). The period ratios of these stars fell on three parallel lines, which were separated by about 0.02 in period ratios in the $P / P_{1 \mathrm{O}}-\log P_{10}$ Petersen diagram. In a theoretical work, Dziembowski (2012) concluded that in Cepheids "the only unstable modes that may reproduce the observed period ratio are $f$-modes of high angular degrees $(l=42-50) . " \quad$ This hypothesis predicts very large $\left(35-70 \mathrm{~km} \mathrm{~s}^{-1}\right)$ broadening of the spectral lines of these stars, which has not yet been verified observationally.

RR Lyrae stars are known to be slow rotators (Peterson et al. 1995; Preston \& Chadid 2013) with a microturbulent velocity of about 2-3 $\mathrm{km} \mathrm{s}^{-1}$ (Govea et al. 2014, and references therein), and no significant line broadening of any RR Lyrae stars has been detected previously.

Spectroscopic data of M3 variables were also collected by Hectochelle@MMT (Szentgyörgyi et al. 2011) with 20,000 spectral resolution in the 5150-5300 $\AA$ wavelength range parallel with the photometric observations in 2012. The details of the observations and data reduction process will be published in a separate paper.

Six overtone RRL stars belonged to the targets of the spectroscopic observations. Three of them-the RRc stars V56 and V87, and the RRd star V97-show the $f_{0.61}$ frequency. The others are normal RRc stars (V86 and V107), and one of them, V140, shows strong Blazhko modulation. We have checked the line broadenings of these stars in the pulsation phase near to minimum brightness. Figure 12 shows the line profiles of the Mgb (5183.604 $\AA$ ) absorption line, which is the deepest nonblended line in the spectra of the six RRc/RRd stars, and the best fitting models using the alpha enhanced synthetic spectra $(Z=-1.5)$ of the Asiago stellar library (Munari et al. 2005). The atmospheric parameters $\left(T_{\text {eff }}, \log g\right)$ were estimated from the observed colors assuming $E(B-V)=0.01 \mathrm{mag}$ 


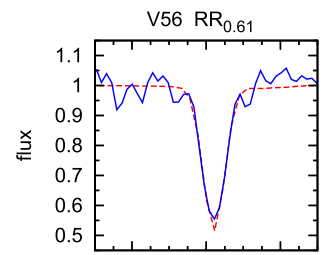

V86 RRc

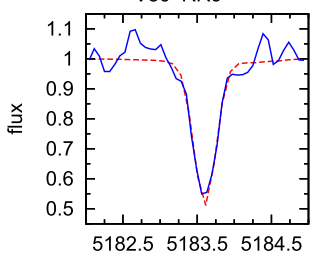

$\lambda[\mathrm{A}]$

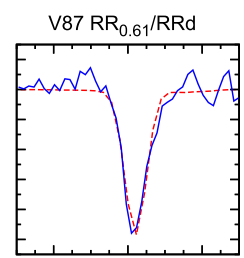

V107 RRc

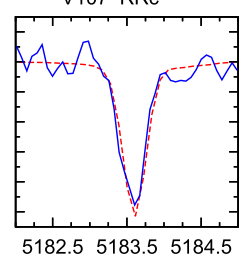

$\lambda[\mathrm{A}]$

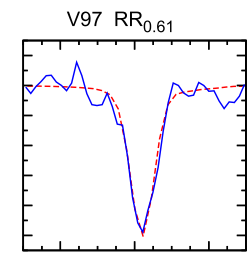

V140 RRc BI

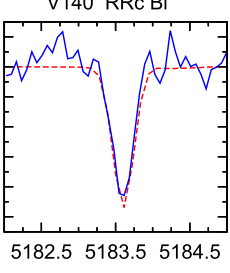

$\lambda[\mathrm{A}]$
Figure 12. The Mgb (5183.604 $\AA$ ) absorption line in the spectra of six RRc/ RRd stars at the pulsation phase around minimum brightness. Stars showing the $f_{0.61}$ frequency are in the top panels. The line profiles are fitted with a synthetic spectra of appropriate physical parameters and of rotational velocities of $5 \mathrm{~km} \mathrm{~s}^{-1}$ ( $\left.v \sin i\right)$ for each star. No systematic difference is detected between the broadenings of the line profiles of $\mathrm{RR}_{0.61}$ and the other $1 \mathrm{O}$ stars.

interstellar reddening using the synthetic colors published in Castelli \& Kurucz (2003). Uncertainties of the physical properties account for about $1-2 \mathrm{~km} \mathrm{~s}^{-1}$ errors of the results.

The broadenings of the line profiles are estimated to be about $5 \mathrm{~km} \mathrm{~s}^{-1}$ for all six stars (Figure 12). The line broadening of the three $R_{0.61}$ stars are thus the same as for RRc stars not showing the $f_{0.61}$ component. The lack of any systematic difference between the broadenings of the line profiles of $\mathrm{RR}_{0.61}$ and other $1 \mathrm{O} \mathrm{RRL}$ stars in M3 contradicts the highorder $f$-mode explanation of the $f_{0.61}$ frequency proposed by Dziembowski (2012).

Amplitude and phase variations of the $f_{0.61}$ component on a timescale of 10-200 days was detected in the Kepler data (Moskalik et al. 2015). However, the Kepler data revealed very low amplitude (tenths of mmag) variations of the radial mode on similar timescale, as well. Such variations are below the detection limit of the D12 data.

The light curves of the Kepler RRc stars with the $f_{0.61}$ frequency component are also sinusoidal or show only a marginal bump (see Figure 2 in Moskalik et al. 2015), as in M3. Although no $\mathrm{H}$ emission in the pre-maximum phase of RRc stars has been reported, the pre-maximum bump in RRab stars is related to a strong atmospheric shock (Gillet \& Fokin 2014, and references therein). The lack of a pronounced bump on the light curves of $\mathrm{RR}_{0.61}$ stars might indicate that the formation of atmospheric shocks and the excitation of the $f_{0.61}$ frequency are mutually exclusive.

The amplitude ratio of the $f_{0.61}$ frequency relative to the amplitude of the radial mode is in the 0.02-0.08 range in M3. The amplitude ratios are in a similar range in all other samples of stars showing the $f_{061}$ frequency. They are $0.01-0.04$, 0.02-0.05, and 0.02-0.08 in Cepheids (Soszyński et al. 2010), the RRc stars observed by the Kepler space telescope (Moskalik et al. 2015), and the Galactic bulge (Netzel et al. 2015), respectively. The $f_{0.61}$ frequency is thus a relatively small-amplitude signal, according to each of these studies.

Each of the four RRc stars observed by the Kepler mission shows the $f_{0.61}$ frequency, moreover, the PD of this component is detected in these stars (Moskalik et al. 2015). The half-
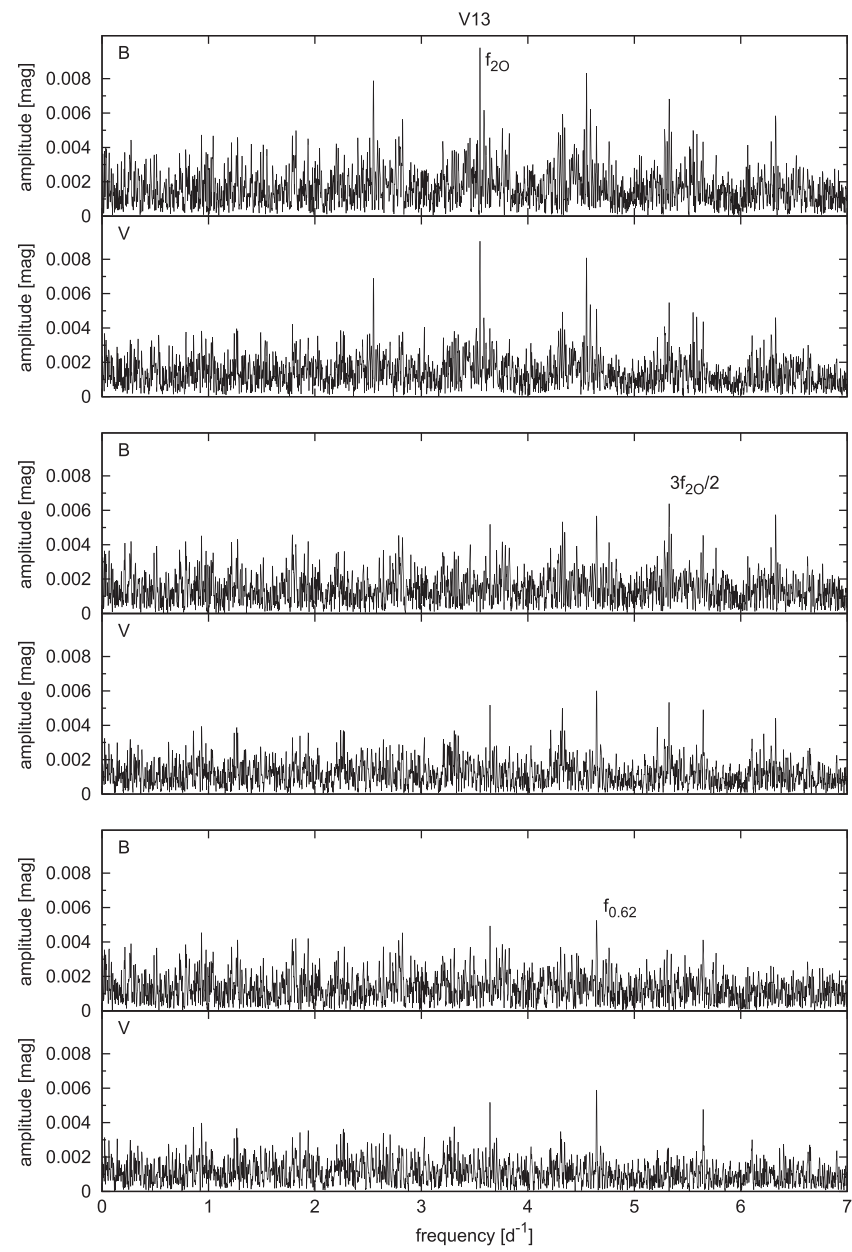

Figure 13. $B$ and $V$ residual spectra of V13 in consecutive prewhitening steps. The largest signal appears at the $f_{2 \mathrm{O}}(2 \mathrm{O}$ mode $)$ frequency in the data prewhitened for the $f_{\mathrm{F}}$ and $f_{1 \mathrm{O}}$ frequency components, their modulations, and the linear-combination terms (top panels). In the next prewhitening steps, frequencies are detected at $3 f_{20} / 2$, and then, at $f_{0.61}$ (middle and bottom panes).

integer frequency of the $f_{0.61}$ component also appears in four of the 147 Galactic bulge $\mathrm{RR}_{0.61}$ stars (Netzel et al. 2015). The amplitudes of these half-integer frequencies are smaller than $0.003 \mathrm{mag}$ with one exception-the amplitude of the $f_{0.61} / 2$ frequency of KIC 5520878 is $0.006 \mathrm{mag}$. No $k f_{0.61} / 2$ halfinteger frequency is detected in M3, however, signals with amplitude smaller than 0.004 mag are below the detection limit of the D12 data.

The $f_{0.61}$ frequency is detected in $38 \%$ of the RRc/RRd stars in M3. Moskalik et al. (2015) found that almost every RRc and RRd star observed from space showed the $f_{0.61}$ frequency, consequently the phenomenon must be very common. Concerning Cepheids, $\sim 9 \%$ of the 10 pulsators showed the $f_{0.61}$ frequency in the SMC (Soszyński et al. 2010), but none of the shortest period ones $\left(\log P_{10}<0.056\right)$ noticed by Dziembowski (2012). It seems that in this sample there is a tendency for the phenomenon to not occur in the hottest stars.

About $20 \%$ of the $\mathrm{RR}_{0.61}$ stars are bona-fide double-mode pulsators in M3. There are four and two RRd stars in the samples of 23 field and GC (Moskalik et al. 2015), and 147 Galactic bulge $R_{0.61}$ stars (Netzel et al. 2015), respectively. Thus the frequency of double-mode pulsators in these data sets is $17 \%$ and $1.4 \%$. The very low rate of RRd stars showing the 
Table 6

Period Doubling Detected in the M3 Data

\begin{tabular}{lcccccc}
\hline \hline Star & Mode $^{\mathrm{a}}$ & $k^{\mathrm{b}}$ & $P($ days $)$ & Error & $P_{\mathrm{DD}}-2 P / k$ & $A(V)$ \\
\hline V13 & $2 \mathrm{O}$ & $\ldots$ & 0.28160 & 0.00003 & $\ldots$ & 0.010 \\
& $\cdots$ & 3 & 0.18775 & 0.00003 & 0.00002 & 0.005 \\
V166 & $\mathrm{F}$ & & 0.48504 & 0.00001 & $\ldots$ & 0.200 \\
& $\cdots$ & 7 & 0.13861 & 0.00002 & 0.00003 & 0.008 \\
\hline
\end{tabular}

Notes.

${ }^{a}$ Pulsation mode period doubling belongs to.

${ }^{\mathrm{b}} \operatorname{Order}(k)$ of the half-integer frequency, $2 P / k$.

$f_{0.61}$ frequency in the Galactic bulge is a consequence of the fact that the occurrence rate of RRd stars among overtone variables in the Galactic bulge (Soszyński et al. 2011) is only $\sim 2 \%$.

The homogeneity of GC data allows us to determine the positions of $\mathrm{RR}_{0.61}$ stars relative to other overtone variables using their measured parameters. Each RRc star close to the blue edge of the double-mode region-based on their periods and colors-as well as four RRd stars, show the $f_{0.61}$ frequency. It is also detected in some highly evolved, bright, long-period variables, whose temperature (indicated by their colors) falls in the temperature range covered by the other $\mathrm{RR}_{0.61}$ stars. The similarity of the light-curve shapes of $\mathrm{RR}_{0.61}$ and $\mathrm{RRd}$ stars (with reduced or missing bump) and their physical parameters (based on the colors and periods) indicates that the $f_{0.61}$ phenomenon could be strongly connected to the double-mode state. It is possible that the complex dynamics of the observed and/or hidden double-mode pulsation are behind the excitation of this peculiar frequency component.

\section{PERIOD DOUBLING}

A new feature of the pulsation of RRab stars, the alternating shapes of their light curves (i.e., PD) was recognized, thanks to the high-precision time-resolved photometry of the Kepler space telescope data (as a review see Szabó 2014). Half-integer frequencies of the fundamental-mode frequency are the signature of the phenomenon in the Fourier spectrum. The observed half-integer frequencies had the highest amplitude, typically at $3 f_{\mathrm{F}} / 2$, and they were detected exclusively in stars showing Blazhko modulation in the Kepler data. Moskalik et al. (2015) reported that half-integer frequencies also appear in RRc stars; however, in this case the PD was connected to the additional $f_{0.61}$ frequency, instead of the radial-mode pulsation component. PD of the third (third radial overtone or non-radial) mode of OGLE-BLG-RRLYR-24137 was also reported by Smolec et al. (2015b). Using turbulent, convective hydrodynamical models and amplitude equation formalism, Kolláth et al. (2011) and Buchler \& Kolláth (2011) argued that a 9:2 resonance between the fundamental and the ninth radial overtone (which is a strange mode) is responsible for the observed PD of RRab stars, and it might have taken part in the generation of the Blazhko modulation. In contrast to this claim, citing Moskalik et al. (2015), "it is unclear which (if any) resonance is responsible for the $\mathrm{PD}$ in the $\mathrm{RRc}$ and $\mathrm{RRd}$ variables."

The D12 data revealed PD of two multi-mode RR Lyrae stars in M3, as well. It is connected to the second overtone of the triple-mode star, V13, and to the fundamental mode of the
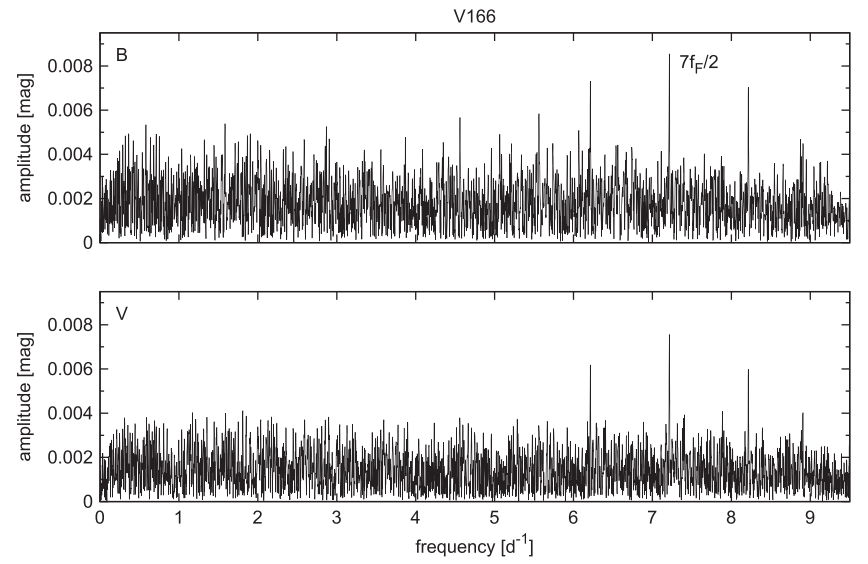

Figure 14. $B$ and $V$ residual spectra of V166, prewhitened for the $f_{\mathrm{F}}$ and $f_{10}$ frequency components and their modulations and linear combinations, are drawn. Frequency appears at the position of the half-integer frequency, $7 f_{\mathrm{F}} / 2$.

double-mode star, V166. Both stars show Blazhko modulation of the fundamental and the overtone modes. Table 6 gives the main properties of the frequencies connected to PD of these stars. These frequencies appear at the exact value of the halfinteger frequencies within the uncertainty limits $(3 \sigma)$.

The $B$ and $V$ residual spectra-prewhitened for the $f_{\mathrm{F}}$ and $f_{1 \mathrm{O}}$ frequency components, their modulations, and the linearcombination terms - are shown for V13 and V166 in Figures 13 and 14 , respectively. The frequency of the $2 \mathrm{O}$ appears first in $\mathrm{V} 13$, then, in further prewhitening steps, frequencies are detected at $3 f_{2 \mathrm{O}} / 2$, and finally, at $f_{0.61}$. The prewhitened spectra of V166 show a signal at the $7 f_{\mathrm{F}} / 2$ half-integer frequency with a high significance.

Despite the fact that the amplitude of the $2 \mathrm{O}$ pulsation mode is only $0.010 \mathrm{mag}$, it is quite surprising that the half-integer frequency is connected to the $2 \mathrm{O}$ mode and not to the most strongly modulated large-amplitude fundamental mode. The amplitude ratio of the half-integer frequency to the $2 \mathrm{O}$ is quite large: $A_{3 f_{20} / 2} / A_{f_{20}}=0.50$. We note that even larger amplitude ratio of any half-integer component was detected only in some of the $f_{0.61}$ frequencies reported by Moskalik et al. (2015) and Netzel et al. (2015). The half-integer frequency was also connected to a small-amplitude frequency component and not the large-amplitude radial mode in these cases.

Looking for a candidate resonance behind the $\mathrm{PD}$ of the $2 \mathrm{O}$ mode of V13, a low-order one, $3 f_{2 \mathrm{O}}=2 f_{4 \mathrm{O}}$, may be valid in the 6800-6900 K temperature range based on the period-ratio grid of radial modes (Figure 3 in Kolláth et al. 2011). According to the temperature calibration of Cacciari et al. (2005), doublemode stars fall exactly into this temperature range in M3. However, as the fourth overtone is strongly damped (see Figure 4 in Kolláth et al. 2011), its excitation, even in resonance position is dubious. Why the PD of the small-amplitude $2 \mathrm{O}$ appears in $\mathrm{V} 13$ and whether its $3 f_{2 \mathrm{O}} / 2$ frequency can be explained by resonances with some high-order overtone remain questions to be answered. Besides the PD feature observed in RRab stars that V166 seems to be following, the behavior of $\mathrm{V} 13$ is pointing to some additional mechanism also taking part in this effect.

PD of the overtone mode of single or double-mode stars or the $f_{0.61}$ frequency component is not detected in M3. 
Table 7

Blazhko Properties According to the D12 Data

\begin{tabular}{|c|c|c|c|c|c|c|}
\hline Star & $\begin{array}{c}P_{\text {puls }} \\
\text { (days) }\end{array}$ & $\begin{array}{l}P_{\text {mod }}{ }^{\mathrm{a}} \\
\text { (days) }\end{array}$ & $\begin{array}{c}f_{\text {mod }} \\
\left(\text { day }^{-1}\right)\end{array}$ & $\begin{array}{c}A_{\mathrm{A}_{\text {mod }}}{ }^{\mathrm{b}} \\
(\mathrm{mag})\end{array}$ & $\begin{array}{c}A_{\mathrm{ph}_{\text {mod }}}{ }^{\mathrm{c}} \\
(\mathrm{rad})\end{array}$ & Summary of the Main Modulation Components \\
\hline \multirow[t]{2}{*}{$\overline{\mathrm{V} 13}$} & 0.35072 & $139(1)$ & 0.007 & 0.20 & 1.25 & $-f_{\mathrm{m}},-2 f_{\mathrm{m}}$ at the first and second harmonic orders \\
\hline & 0.47949 & $139(1)$ & 0.007 & 0.42 & 0.85 & dominant $-f_{\mathrm{m}}$, some small amplitude $+f_{\mathrm{m}},-2 f_{\mathrm{m}}$ in the first 4 harmonic orders \\
\hline \multirow[t]{2}{*}{ V44 } & 0.36812 & $56.0(8)$ & 0.018 & 0.15 & 2.20 & complex multiplets at the first and second harmonic orders \\
\hline & 0.50377 & $97.0(2)$ & 0.010 & 0.45 & 1.00 & complex modulation with strong $-f_{\mathrm{m}},-2 f_{\mathrm{m}}$ and $\pm f_{\mathrm{m}} / 2$ components \\
\hline V70 & 0.48607 & $150(10)$ & 0.007 & 0.01 & 0.10 & triplet with 0.007 and $0.006 \mathrm{mag}$ amplitude of the $-f_{\mathrm{m}},+f_{\mathrm{m}}$ components \\
\hline \multirow[t]{2}{*}{ V99 } & 0.3611 & $40.3(2)$ & 0.025 & 0.35 & 2.20 & $+f_{\mathrm{m}},+2 f_{\mathrm{m}},+3 f_{\mathrm{m}}$ components at the first and second harmonic orders \\
\hline & 0.4821 & $\approx 450$ & $\approx 0.002$ & $>0.25$ & $>0.2$ & triplet with 0.050 and $0.025 \mathrm{mag}$ amplitude of the $-f_{\mathrm{m}}$ and $+f_{\mathrm{m}}$ components \\
\hline V126 & 0.34841 & $15.0(2)$ & 0.067 & 0.03 & 0.04 & one component at $f+f_{\mathrm{m}}$ with $0.006 \mathrm{mag}$ amplitude \\
\hline V140 & 0.33316 & $28.8(1)$ & 0.035 & 0.10 & 0.63 & triplets at least up to the 5 th harmonic order \\
\hline \multirow[t]{2}{*}{ V166 } & 0.35672 & $43.5(5)$ & 0.023 & 0.08 & 0.50 & one component at $f-f_{\mathrm{m}}$ with $0.023 \mathrm{mag}$ amplitude \\
\hline & 0.48504 & $71.5(3)$ & 0.014 & 0.45 & 1.20 & members of the quintuplets (dominant $-f_{\mathrm{m}},-2 f_{\mathrm{m}}$ ) up to the 4 th harmonic order \\
\hline V168 & 0.27594 & $23.0(1)$ & 0.043 & 0.16 & 1.00 & members of the quintuplets up to the 4 th harmonic order \\
\hline V203 & 0.28979 & $118(5)$ & 0.008 & 0.03 & 0.15 & one component at $f+f_{\mathrm{m}}$ with $0.007 \mathrm{mag}$ amplitude \\
\hline
\end{tabular}

Notes.

a The error in the last digit is given in parenthesis.

b Full amplitude of the amplitude modulation in the $V$ band.

${ }^{\mathrm{c}}$ Full amplitude of the phase modulation in the $V$ band.
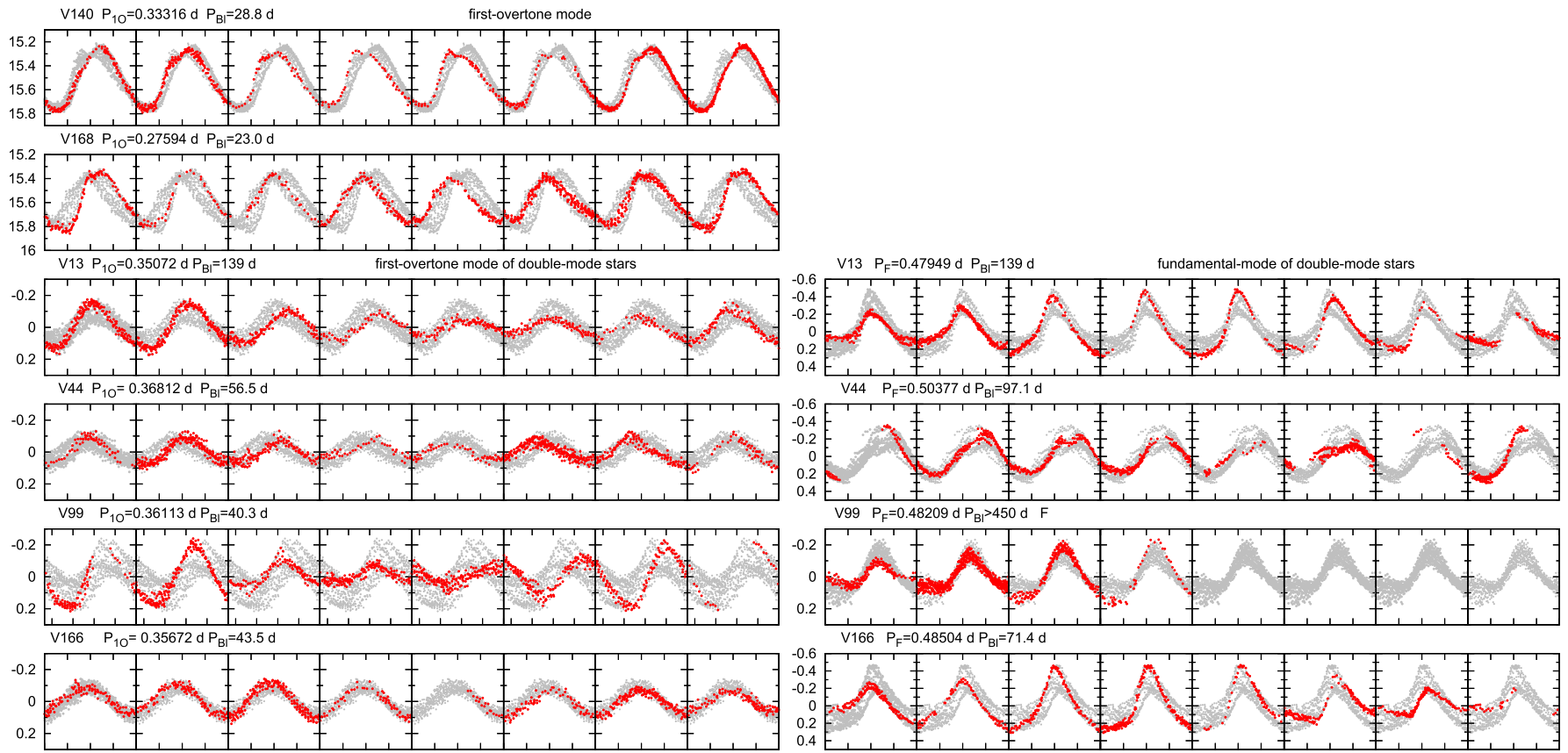

Figure 15. The $V$ light curves of the Blazhko stars in eight phases of their main modulation cycle are shown. The light curves of the double-mode stars are prewhitened for the light variation of the other mode and for the linear-combination terms.

\section{BLAZHKO EFFECT}

Equally spaced asymmetric triplets or multiplets, which are characteristics for Blazhko RRab stars, are detected in the residual spectra of seven overtone and double-mode stars. A small-amplitude component near the radial-mode frequency appears in the residual spectra of two further stars (V126, V203). Although the non-radial mode origin of these latter signals cannot be excluded, we also classify these stars as Blazhko variables. Six of the Blazhko stars are multiperiodic aside from their light-curve modulation. V13, V44, V99, and
V166 are double(triple)-mode stars, and the $f_{0.61}$ frequency component is detected in V70 and V126. Some specialties of the Blazhko properties of overtone stars in M3 were already discussed in Jurcsik et al. (2014). The Blazhko stars are denoted by thick symbols in Figures 3 and 4 .

Table 7 lists the pulsation and modulation periods, the amplitudes of the amplitude- and phase-modulations, and data and comments on the main features of the modulation detected in the D12 data of Blazhko stars.

The light curves of the strongly modulated stars in eight phases of the Blazhko cycle are shown in Figure 15. The data 
of multi-mode pulsators are prewhitened for the signals of the pulsation and the modulation of the other radial mode, the $f_{0.61}$ frequency, and for the linear-combination terms. Note that the modulation periods of the two modes are different in V44, V99, and V166.

The light curves in Figure 15 and the data given in Table 7 show that the modulation of RRc and the overtone mode of RRd stars are phase modulations predominantly. The phase modulation of the overtone mode of V44 and V99 is larger than one-third of the pulsation period. This is in contrast with the Blazhko behavior of the fundamental mode of RRd and RRab stars. Their modulation is dominated mostly by amplitude variation, or their amplitude and phase modulations have similar strength.

A pre-maximum bump only appears in the two single-mode Blazhko stars (V140 and V168) in some phases of their Blazhko cycle. Both of these stars are at high luminosity, but at different temperatures as indicated by their magnitudes, periods, and color indices (see Figure 3). The bump is observed at the opposite phases of the modulation of the two stars. The shock-induced phenomenon appears in V140 and V168 when the phase shift is negative (period is decreasing) and the amplitude is small, and when the phase-shift of the light curve is positive (period is increasing) and the amplitude is large, respectively. The temperature difference between the two stars may explain this reverse behavior. The temporal appearance of the bump in Blazhko RRc stars implies that their main physical properties vary during the Blazhko cycle, similarly as detected in Blazhko RRab stars (see, e.g., Jurcsik et al. 2008, 2012b; Sódor et al. 2011).

\subsection{Notes on the Blazhko Properties of the Stars}

Besides the analysis of the D12 data, the archive photographic and CCD data collected in J12 have also been checked to follow the changes of the Blazhko properties on a long timescale. Further details on the pulsation/modulation of Blazhko RRd stars are given in Section 7.

$V 13$ is a triple-mode star $(\mathrm{F} / 1 \mathrm{O} / 2 \mathrm{O})$ with a dominant fundamental mode (Section 7.1). The $\mathrm{F}$ and 10 modes are modulated with the same period, and the amplitude and phase variations of the modes are in anti-phase with each other (see also in Jurcsik et al. 2014), similarly to 10/2O Blazhko Cepheids (Moskalik \& Kołaczkowski 2009). The amplitude ratios of the radial modes to the largest amplitude modulation components are 2.3 and 1.6 for the $\mathrm{F}$ and 10 modes. Assuming that the pulsation/modulation amplitude ratios of each radial mode are similar, the modulation amplitudes are expected to be around $0.004-0.005 \mathrm{mag}$ at the $2 \mathrm{O}$ frequency, because the $V$ amplitude of the $2 \mathrm{O}$ mode is $0.009 \mathrm{mag}$. However, no modulation component with amplitude larger than $\sim 0.002$ mag has been found; the largest amplitude signal close to the 20 frequency in the residual spectrum is the 1 day $^{-1}$ alias component of the $f_{0.61}$ frequency (see Figure 13). Previous CCD observations show a similar behavior as in the D12 data; anti-phase modulations of the fundamental and overtone modes with 144 days is derived from the combined K98, B06, and J12 data set.

V44 is a double-mode star with a dominant fundamental mode. The modulation of the fundamental mode is very complex; the main modulation period is 97 days but modulations with half and twice this period are also detected. The 56 day modulation of the overtone mode is dominantly phase
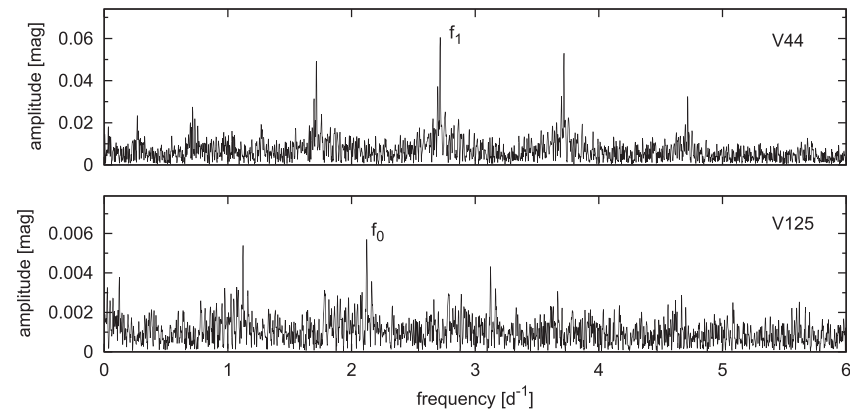

Figure 16. Residual spectra of the detected new radial-mode components of V44 and V125. The data are prewhitened for all the other signals.

modulation. Modulation of the fundamental mode with 52, 104, and 111 days are identified in the C01, H05+B06, and the J12 data, respectively. The photographic data show some signs of modulation with $P_{\mathrm{m}}=82$ days (JD 2420625-2424684), and 49 days (JD 2428963-2436991).

$V 70$ is a very-long-period (0.486 days), over-luminous, evolved variable. The small-amplitude, 150 day modulation detected in the D12 data is dominantly phase modulation, but amplitude changes are also observed mostly in the $B$ band. The amplitude changes and the 150 day period of the modulation, which is shorter than the time span of the observations, make it unlikely that period change mimics Blazhko modulation in this case. The C01, H05, B06, and J12 data show significant residuals at $f_{10}$ but with different separations. However, it is not clear in these cases whether these signals arise from strong period changes, which are typical in this star, or from lightcurve modulation.

V99 is a double-mode star. The amplitude of the fundamental mode was increasing from 0.13 to $0.42 \mathrm{mag}$ in the D12 data. The $V$ amplitude of the fundamental mode was $0.20,0.40$, and $0.25 \mathrm{mag}$ in 1992-1993, 1999, and 2009, respectively, according to previous CCD observations (C01, H05, B06, and J12). The strong differences in the measured amplitudes of the fundamental mode indicates that the amplitude increase of the fundamental mode in the D12 data reflects Blazhko modulation on a timescale of some hundred days and that the true amplitude of the fundamental mode can be even larger as measured in the D12 data. The overtone mode is strongly amplitude and phase modulated. Blazhko modulation of the overtone mode is identified in both the combined H05 and B06 and the J12 data with a period of around 40 days.

V126 is an RRc star showing a small-amplitude $f_{1 \mathrm{O}}+f_{\mathrm{m}}$ component. This RRc star is the longest period, not overluminous one in the magnitude calibrated sample, which lies just at the blue edge of the double-mode region.

V140 is about 0.2 mag above the zero-age horizontal branch. The $\mathrm{J} 12$ data show Blazhko modulation $\left(f_{1 \mathrm{O}}+f_{\mathrm{m}}, f_{1 \mathrm{O}}-f_{\mathrm{m}}\right.$, and $2 f_{1 \mathrm{O}}-f_{\mathrm{m}}$ components) with $f_{\mathrm{m}}=0.0689 \mathrm{day}^{-1}$ $\left(P_{\mathrm{m}}=14.5\right.$ days $)$, which is half the recent modulation period. The combined H05 and B06 observations yield similar lightcurve modulation as observed in the D12 data. The pulsation and modulation periods were 0.333146 and 29.0 days in 1999 . The modulation period cannot be determined in the $\mathrm{C} 01$ data, but from the large residuals around the pulsation frequency we suppose that the light curve was also modulated between JD 2448755 and 2449091. Similar modulation as observed in the CCD data is suspected in two parts of the photographic data; the $f_{1 \mathrm{O}}+0.068$ day $^{-1}(\sim 15$ days $)$ component could be present 
Table 8

Long-term Changes in the Properties of Double-mode Stars According to a Reanalysis of the Photometric Data

\begin{tabular}{|c|c|c|c|c|c|c|c|c|c|c|c|}
\hline Star & Band & JD-2400000 & $\begin{array}{c}f_{\mathrm{F}} \\
\left(\mathrm{day}^{-1}\right)\end{array}$ & $\begin{array}{c}A_{\mathrm{F}}{ }^{\mathrm{a}} \\
(\mathrm{mag})\end{array}$ & $\begin{array}{c}f_{1 \mathrm{O}} \\
\left(\text { day }^{-1}\right)\end{array}$ & $\begin{array}{c}A_{10} \\
(\mathrm{mag})\end{array}$ & $A_{\mathrm{F}} / A_{1 \mathrm{O}}$ & $f_{\mathrm{F}} / f_{1 \mathrm{O}}$ & $\begin{array}{c}f_{\mathrm{mF}}^{\mathrm{b}} \\
\left(\mathrm{day}^{-1}\right)\end{array}$ & $\begin{array}{c}f_{\mathrm{m} 10^{\mathrm{c}}} \\
\left(\mathrm{day}^{-1}\right)\end{array}$ & Remark \\
\hline \multirow[t]{6}{*}{ V13 } & $\mathrm{pg}$ & $23858-24684$ & 2.07019 & 0.46 & $\cdots$ & $\cdots$ & $\cdots$ & $\cdots$ & $\cdots$ & $\ldots$ & $\ldots$ \\
\hline & $\mathrm{pg}$ & $28963-35933$ & 2.07022 & 0.36 & $\cdots$ & $\cdots$ & $\cdots$ & $\cdots$ & $\cdots$ & $\cdots$ & $\cdots$ \\
\hline & $\mathrm{pg}$ & $36367-37131$ & 2.07023 & 0.38 & $\cdots$ & $\cdots$ & $\cdots$ & $\cdots$ & $\cdots$ & $\cdots$ & $\cdots$ \\
\hline & $\mathrm{pg}$ & $39944-47554$ & 2.08544 & 0.28 & $\cdots$ & $\cdots$ & $\cdots$ & $\cdots$ & $\cdots$ & $\cdots$ & $\cdots$ \\
\hline & CCD $V$ & $50162-54965$ & 2.08555 & 0.22 & 2.85123 & 0.07 & 3.3 & 0.7315 & 0.0069 & 0.0069 & $\cdots$ \\
\hline & CCD $V$ & $55935-56137$ & 2.08555 & 0.23 & 2.85127 & 0.08 & 2.9 & 0.7314 & 0.0072 & 0.0072 & $f_{2 \mathrm{O}}, f_{\mathrm{PD}}=3 f_{2 \mathrm{O}} / 2, f_{0.61}$ \\
\hline \multirow[t]{8}{*}{ V44 } & pg & $13372-22840$ & 1.97485 & 0.58 & $\ldots$ & $\ldots$ & $\ldots$ & $\ldots$ & $\ldots$ & $\ldots$ & $\ldots$ \\
\hline & $\mathrm{pg}$ & $24283-30078$ & 1.97490 & 0.54 & $\ldots$ & $\cdots$ & $\cdots$ & $\ldots$ & $\ldots$ & $\ldots$ & $\ldots$ \\
\hline & $\mathrm{pg}$ & $31965-36991$ & 1.97471 & 0.45 & $\ldots$ & $\cdots$ & $\ldots$ & $\ldots$ & $\ldots$ & $\ldots$ & $\ldots$ \\
\hline & $\mathrm{pg}$ & $37018-47555$ & 1.97470 & 0.30 & $\ldots$ & $\cdots$ & $\ldots$ & $\ldots$ & $\ldots$ & $\ldots$ & $\ldots$ \\
\hline & CCD $V$ & $48755-49091$ & 1.975: & 0.22 & 2.7: & 0.06 & 3.7 & $0.731:$ & $0.019:$ & $\ldots$ & $\ldots$ \\
\hline & CCD $V$ & 50893-51257 & 1.9744 & 0.40 & $\ldots$ & $\ldots$ & $\ldots$ & $\ldots$ & $0.010 / 0.019$ & $\ldots$ & $\ldots$ \\
\hline & CCD $V$ & $54869-54965$ & 1.9736 & 0.30 & 2.685: & 0.03 & 10.0 & 0.7350 & $0.009 / 0.018$ & $\cdots$ & $\cdots$ \\
\hline & CCD $V$ & 55935-56137 & 1.98505 & 0.21 & 2.71650 & 0.06 & 3.5 & 0.7307 & $0.005 / 0.010 / 0.020$ & 0.018 & $\cdots$ \\
\hline \multirow[t]{6}{*}{ V68 } & $\mathrm{pg}$ & $22455-24684$ & 2.08989 & 0.25 & 2.80916 & 0.26 & 0.8 & 0.7440 & $\cdots$ & $\cdots$ & $\cdots$ \\
\hline & $\mathrm{pg}$ & 33034-39944 & 2.08985 & 0.22 & 2.80920 & 0.25 & 0.8 & 0.7439 & $\cdots$ & $\cdots$ & $\cdots$ \\
\hline & CCD $V$ & $48755-50176$ & 2.08985 & 0.17 & 2.80921 & 0.20 & 0.8 & 0.7439 & $\ldots$ & $\cdots$ & $\ldots$ \\
\hline & CCD $V$ & 50893-51257 & 2.08988 & 0.17 & 2.80918 & 0.19 & 0.9 & 0.7439 & $\ldots$ & $\cdots$ & $\ldots$ \\
\hline & CCD $V$ & $54869-54965$ & 2.0899 & 0.17 & 2.8092 & 0.21 & 0.8 & 0.7440 & $\ldots$ & $\ldots$ & $\ldots$ \\
\hline & CCD $V$ & $55935-56137$ & 2.08986 & 0.17 & 2.80921 & 0.20 & 0.9 & 0.7439 & $\cdots$ & $\cdots$ & $f_{0.61}$ \\
\hline \multirow[t]{16}{*}{ V79 } & pg & $13372-20656$ & 2.0666 & 0.66 & $\cdots$ & $\cdots$ & $\cdots$ & $\cdots$ & $\cdots$ & $\cdots$ & $\cdots$ \\
\hline & $\mathrm{pg}$ & $22729-24684$ & 2.0697 & 0.51 & $\cdots$ & $\cdots$ & $\cdots$ & $\cdots$ & $\cdots$ & $\cdots$ & $\cdots$ \\
\hline & pg & $28963-35933$ & 2.0690 & 0.50 & $\ldots$ & $\cdots$ & $\cdots$ & $\cdots$ & 0.050: & $\cdots$ & $\cdots$ \\
\hline & pg & $36367-39672$ & 2.0691 & 0.44 & $\cdots$ & $\cdots$ & $\cdots$ & $\cdots$ & 0.012: & $\cdots$ & $\cdots$ \\
\hline & $\mathrm{pg}$ & $41061-42970$ & 2.0698 & 0.25 & $\cdots$ & $\cdots$ & $\cdots$ & $\cdots$ & $\cdots$ & $\cdots$ & residual at $f_{\mathrm{F}}$ \\
\hline & $\mathrm{pg}$ & $43160-44760$ & 2.0691 & 0.42 & $\ldots$ & $\cdots$ & $\ldots$ & $\ldots$ & $\ldots$ & $\cdots$ & residual at $f_{\mathrm{F}}$ \\
\hline & $\mathrm{pg}$ & $44988-46566$ & 2.0696 & 0.43 & $\ldots$ & $\ldots$ & $\ldots$ & $\ldots$ & $\ldots$ & $\ldots$ & $\ldots$ \\
\hline & pg & $47202-47329$ & 2.0711 & 0.49 & $\ldots$ & $\ldots$ & $\ldots$ & $\ldots$ & $0.015:$ & $\ldots$ & signal at $2.087 \mathrm{day}^{-1}$ \\
\hline & pg & $47552-47686$ & $\ldots$ & $\ldots$ & $\ldots$ & $\ldots$ & $\ldots$ & $\ldots$ & $\ldots$ & $\ldots$ & at JD 2447620 pulsation ceases \\
\hline & pg & $47970-48485$ & 2.0843 & 0.29 & & $\ldots$ & $\ldots$ & $\ldots$ & $\ldots$ & $\ldots$ & strong residual at $2.0743 \mathrm{day}^{-1}$ \\
\hline & CCD $V$ & 4875549091 & $2.0856:$ & 0.11 & 2.7931: & 0.16 & 0.7 & 0.7467 & $\ldots$ & $\ldots$ & $\ldots$ \\
\hline & CCD $V$ & $50162-50176$ & 2.084 & 0.09 & 2.792 & 0.17 & 0.5 & 0.746 & $\ldots$ & $\ldots$ & $\ldots$ \\
\hline & CCD $V$ & 50893-50972 & 2.0865 & 0.09 & 2.7914 & 0.17 & 0.5 & 0.7474 & 0.015 & $\ldots$ & $\ldots$ \\
\hline & CCD clear & $54257-54273$ & 2.072 & 0.32 & 2.795 & 0.13 & 2.5 & 0.741 & $\ldots$ & $\ldots$ & $\ldots$ \\
\hline & CCD $V$ & $54869-54965$ & 2.06913 & 0.41 & & $\ldots$ & $\ldots$ & $\ldots$ & 0.016 & $\ldots$ & largest mod. peak at $2.085 \mathrm{day}^{-1}$ \\
\hline & CCD $V$ & $55935-56137$ & 2.06914 & 0.35 & & $\ldots$ & $\ldots$ & $\ldots$ & $0.006 / 0.016 / 0.055$ & $\ldots$ & third largest mod. peak at $2.085 \mathrm{day}^{-1}$ \\
\hline \multirow[t]{7}{*}{ V87 } & pg & 22729-24684 & 2.08184 & 0.14 & 2.79737 & 0.32 & 0.5 & 0.7442 & $\ldots$ & $\ldots$ & $\ldots$ \\
\hline & pg & 33390-39944 & 2.08261 & 0.09 & 2.79732 & 0.25 & 0.4 & 0.7445 & $\ldots$ & $\ldots$ & $\ldots$ \\
\hline & $\mathrm{pg}$ & $42838-47555$ & 2.08147 & 0.13 & 2.79733 & 0.29 & 0.5 & 0.7441 & $\ldots$ & $\ldots$ & $\ldots$ \\
\hline & CCD $V$ & $48755-50176$ & 2.08248 & 0.08 & 2.79732 & 0.20 & 0.4 & 0.7445 & $\ldots$ & $\ldots$ & $\ldots$ \\
\hline & CCD $V$ & $50893-51283$ & 2.08238 & 0.09 & 2.79738 & 0.20 & 0.5 & 0.7444 & $\ldots$ & $\ldots$ & $\ldots$ \\
\hline & CCD $V$ & 54869-54965 & 2.08263 & 0.09 & 2.79715 & 0.22 & 0.4 & 0.7446 & $\ldots$ & $\ldots$ & $\ldots$ \\
\hline & CCD $V$ & $55935-56137$ & 2.08260 & 0.08 & 2.79729 & 0.19 & 0.4 & 0.7445 & $\ldots$ & $\cdots$ & $f_{0.61}$ \\
\hline \multirow[t]{2}{*}{ V99 } & $\mathrm{pg}$ & $13372-15161$ & $\ldots$ & $\ldots$ & 2.77062 & 0.42 & $\ldots$ & $\ldots$ & $\ldots$ & $\ldots$ & $\ldots$ \\
\hline & $\mathrm{pg}$ & $22455-24684$ & 2.0673: & 0.22 & $2.7712:$ & 0.23 & 0.8 & 0.7460: & $\ldots$ & $\ldots$ & $\ldots$ \\
\hline
\end{tabular}


Table 8

(Continued)

\begin{tabular}{|c|c|c|c|c|c|c|c|c|c|c|c|}
\hline Star & Band & JD-2400000 & $\begin{array}{c}f_{\mathrm{F}} \\
\left(\text { day }^{-1}\right)\end{array}$ & $\begin{array}{c}A_{\mathrm{F}}^{\mathrm{a}} \\
(\mathrm{mag})\end{array}$ & $\begin{array}{c}f_{1 \mathrm{O}} \\
\left(\mathrm{day}^{-1}\right)\end{array}$ & $\begin{array}{c}A_{1 \mathrm{O}} \\
(\mathrm{mag})\end{array}$ & $A_{\mathrm{F}} / A_{1 \mathrm{O}}$ & $f_{\mathrm{F}} / f_{1 \mathrm{O}}$ & $\begin{array}{c}f_{\mathrm{mF}}^{\mathrm{b}} \\
\left(\mathrm{day}^{-1}\right)\end{array}$ & $\begin{array}{c}f_{\mathrm{m}_{10}}^{\mathrm{c}} \\
\left(\text { day }^{-1}\right)\end{array}$ & Remark \\
\hline & pg & $33034-35933$ & 2.0719 & 0.10 & 2.7712 & 0.21 & 0.5 & 0.7476 & $\ldots$ & $\ldots$ & $\ldots$ \\
\hline & pg & $36367-39933$ & 2.0732 & 0.14 & 2.7711 & 0.20 & 0.6 & 0.7482 & $\ldots$ & $\ldots$ & residual at 2.069 \\
\hline & $\mathrm{pg}$ & $42838-43254$ & $\cdots$ & $\cdots$ & 2.7711 & 0.20 & $\cdots$ & $\cdots$ & $\cdots$ & $\cdots$ & $\cdots$ \\
\hline & CCD $V$ & 48755-49091 & 2.0746: & 0.08 & 2.7707: & 0.24 & 0.3 & $0.7488:$ & $\ldots$ & $\cdots$ & $\cdots$ \\
\hline & CCD $V$ & 50893-50972 & 2.0740 & 0.15 & 2.7696 & 0.10 & 1.4 & 0.7488 & $\ldots$ & 0.025 & $\ldots$ \\
\hline & CCD $V$ & 54869-54965 & 2.0734 & 0.10 & 2.7703 & 0.18 & 0.6 & 0.7484 & $\ldots$ & 0.025 & $\ldots$ \\
\hline & CCD $V$ & 55935-56137 & 2.0743 & 0.12 & 2.7691 & 0.09 & 1.4 & 0.7490 & 0.002: & 0.025 & $\cdots$ \\
\hline \multirow[t]{7}{*}{ V125 } & $\mathrm{pg}$ & $13372-24684$ & & $\ldots$ & 2.85862 & 0.36 & $\ldots$ & $\ldots$ & $\cdots$ & $\ldots$ & $\ldots$ \\
\hline & $\mathrm{pg}$ & 28963-39944 & & $\cdots$ & 2.85860 & 0.28 & $\cdots$ & $\ldots$ & $\cdots$ & $\cdots$ & $\cdots$ \\
\hline & $\mathrm{pg}$ & $42838-47555$ & $\ldots$ & $\cdots$ & 2.85859 & 0.26 & $\ldots$ & $\ldots$ & $\cdots$ & $\ldots$ & $\ldots$ \\
\hline & CCD $V$ & $48755-50176$ & & $\cdots$ & 2.85860 & 0.21 & $\cdots$ & $\cdots$ & $\cdots$ & $\cdots$ & $\cdots$ \\
\hline & CCD $V$ & 50893-50972 & $\cdots$ & $\cdots$ & 2.85869 & 0.20 & $\cdots$ & $\cdots$ & $\cdots$ & $\cdots$ & $\cdots$ \\
\hline & CCD $V$ & 54869-54965 & $\cdots$ & $\cdots$ & 2.85857 & 0.21 & $\cdots$ & $\cdots$ & $\cdots$ & $\cdots$ & $\cdots$ \\
\hline & CCD $V$ & 55935-56137 & 2.1233 & 0.01 & 2.85862 & 0.21 & 0.03 & 0.7428 & $\cdots$ & $\cdots$ & $f_{0.61}$ \\
\hline \multirow[t]{4}{*}{ V166 } & CCD $V$ & $48755-50176$ & 2.0619 & 0.18 & 2.8031 & 0.14 & 1.2 & 0.7356 & $\cdots$ & $\cdots$ & strong residual at $f_{\mathrm{F}}$ \\
\hline & CCD $V$ & 50893-51283 & 2.0621 & 0.20 & 2.8031 & 0.09 & 2.3 & 0.7356 & 0.014 & $\cdots$ & $\cdots$ \\
\hline & CCD $V$ & 54869-54965 & 2.0623 & 0.23 & 2.8034 & 0.12 & 1.9 & 0.7356 & 0.014 & $\cdots$ & $\cdots$ \\
\hline & CCD $V$ & $55935-56137$ & 2.0617 & 0.20 & 2.8033 & 0.09 & 2.2 & 0.7355 & 0.014 & 0.023 & $f_{\mathrm{PD}}=7 f_{\mathrm{F}} / 2$ \\
\hline \multirow[t]{3}{*}{ V200 } & CCD $V$ & 50893-51283 & 2.0606 & 0.19 & 2.7700 & 0.18 & 1.0 & 0.7439 & $\ldots$ & $\cdots$ & $\ldots$ \\
\hline & CCD flux $(V)$ & $50920-50965$ & 2.0606 & $\cdots$ & 2.7690 & $\cdots$ & 0.8 & 0.7442 & $\cdots$ & $\cdots$ & $\cdots$ \\
\hline & CCD flux $(B)$ & $55935-56137$ & 2.06058 & $\cdots$ & 2.76995 & $\cdots$ & 0.9 & 0.7439 & $\cdots$ & $\cdots$ & $\cdots$ \\
\hline \multirow[t]{2}{*}{ V251 } & CCD $V$ & $50896-51283$ & 2.0998: & 0.18 & 2.8232: & 0.15 & 1.2 & 0.7438 & $\cdots$ & $\cdots$ & $\cdots$ \\
\hline & CCD flux $(B)$ & $55935-56137$ & 2.0998 & $\cdots$ & 2.8230 & $\cdots$ & 1.0 & 0.7438 & $\cdots$ & $\cdots$ & $\cdots$ \\
\hline \multirow[t]{3}{*}{ V252 } & $\mathrm{CCD} B$ & $48755-50622$ & 2.2071: & 0.28 & 2.9754: & 0.32 & 0.9 & 0.7418 & $\cdots$ & $\cdots$ & $\cdots$ \\
\hline & CCD $V$ & $50893-51283$ & 2.2076 & 0.18 & 2.9755 & 0.19 & 0.9 & 0.7419 & $\cdots$ & $\cdots$ & $\cdots$ \\
\hline & CCD flux $(B)$ & $55935-56137$ & 2.2075 & $\cdots$ & 2.9754 & & 1.0 & 0.7419 & $\cdots$ & $\cdots$ & $\cdots$ \\
\hline
\end{tabular}

Notes.

${ }^{\mathrm{a}}$ Fourier amplitude; $A_{\mathrm{pg}}$ is about 1.3 times larger than $A_{V}$; at the earliest epochs (JD 2413000-2415000) it is about two times larger.

$\mathrm{b}$ Modulation frequency of the $\mathrm{F}$ mode.

${ }^{\mathrm{c}}$ Modulation frequency of the 10 mode. 
in the data between JD 2422729-2424684 and JD 2433390-2435933, in the latter data set the $f_{10}-0.034$ day $^{-1}$ ( 29 days) component also has a significant amplitude. The dominant modulation of V140 seems to switch between $f_{\mathrm{m}}$ and $2 f_{\mathrm{m}}$ time to time.

V166 is a double-mode star with a dominant fundamental mode. The fundamental mode shows strong amplitude and phase modulations, whereas the modulation of the overtone mode is modest. A 71 day modulation of the fundamental mode is also detected in the B06 and J12 data, and the large residual at $f_{\mathrm{F}}$ points to modulation of the fundamental mode in the $\mathrm{C} 01$ data, too.

V168 is a short period, over-luminous star. The combined H05 and B06 data yield similar light-curve modulation as observed in the D12 data. The pulsation and modulation periods were 0.27595 and 22.9 days in 1999. Modulation of the fundamental mode is also evident in the $\mathrm{C} 01$ data.

$V 203$ is a second-overtone over-luminous variable. A frequency component at $f_{2 \mathrm{O}}+0.0096 \mathrm{day}^{-1}$ is also detected in the $\mathrm{J} 12$ data with 0.008 mag amplitude.

\section{DOUBLE- AND TRIPLE-MODE STARS}

Nine double-mode stars have previously been identified in M3. A thorough investigation of M3 RRd stars was given by (Clementini et al. 2004, hereafter C04). The double-mode nature of two more stars, V44 and V125, and the triple-mode behavior of $\mathrm{V} 13$ are detected in the D12 data. The residual spectra prewhitened for all the other signals in Figure 16 depict the new radial modes of V44 and V125 in the D12 data, similar to the $2 \mathrm{O}$ mode of V13 in Figure 13.

The D12 data makes it possible to reveal the complex behavior of RRd stars. To inspect the changes of pulsation properties, all previous photometric data listed in Section 2 are also checked. The 200 days length of the D12 data allows us to determine the pulsation frequencies of double-mode stars, typically with a precision of the order of $10^{-4}-10^{-5}$ day $^{-1}$. The frequency spectra of the previous photometric data are strongly biased; especially the $1 \mathrm{yr}^{-1}$ alias frequencies, which result in $\pm 0.003 \mathrm{day}^{-1}$ ambiguities of the solutions appear. Therefore, the frequencies published in the literature (K98, C01, $\mathrm{C} 04$, H05, B06, and J12) were revised in the sense that from the equally possible frequency solutions we accepted the one that gave the closest resemblance to the solution of the D12 data.

The main photometric properties of the double/triple-mode stars obtained from the D12 data are given in Table 4. Table 8 summarizes the detected pulsation and modulation (discussed in Section 6) properties of double-mode stars at different epochs.

\subsection{The Triple Mode Star, V13}

The new frequency components detected in the residual spectra of V13 are shown in Figure 13. The frequency appearing at $f_{\mathrm{F}} / f_{x}=0.5873\left(f_{1 \mathrm{O}} / f_{x}=0.8028\right)$ frequency ratio matches the required $P_{2} / P_{0}$ period-ratio range $(0.582-0.593)$ of F+2O double-mode RR Lyrae-type stars (Moskalik 2014). Therefore, we identify this signal with the second radial overtone mode. The radial-mode frequencies are far from the $2 f_{1 \mathrm{O}}=f_{\mathrm{F}}+f_{2 \mathrm{O}}$ resonance condition, $2 f_{1 \mathrm{O}}-f_{2 \mathrm{O}}=2.1508$, while $f_{10}$ is observed at 2.0855 day $^{-1}$.

Modeling the radial-mode frequencies of triple-mode stars provides a unique opportunity to determine the physical parameters of the stars precisely. Unfortunately, pulsation

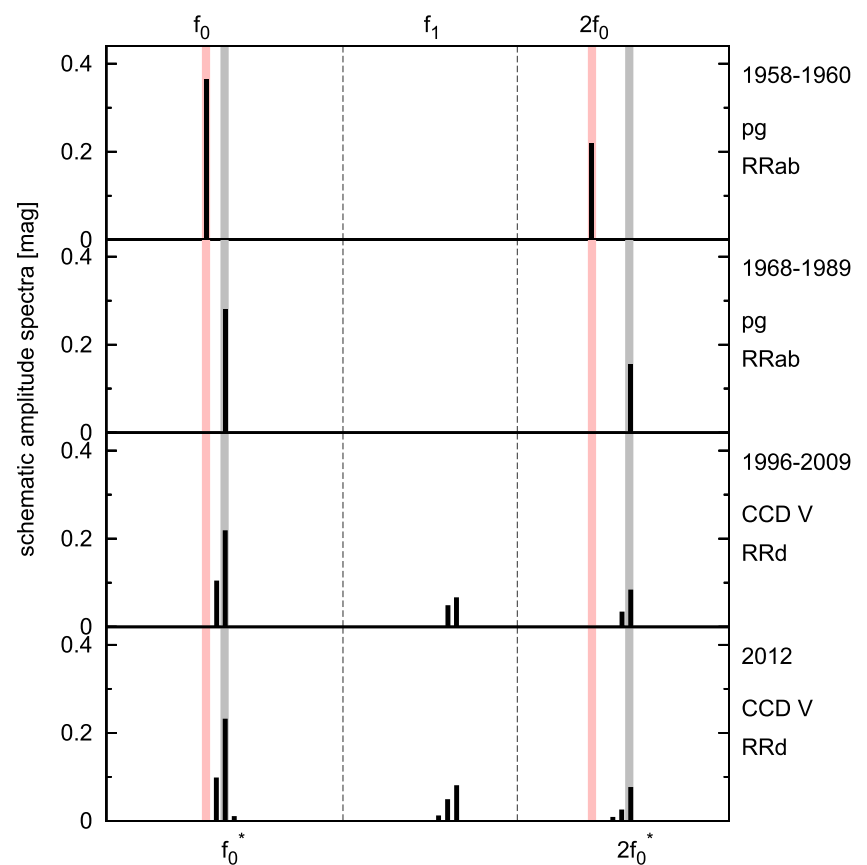

Figure 17. Evolution of the $f_{\mathrm{F}}, 2 f_{\mathrm{F}}$, and $f_{1 \mathrm{O}}$ frequency components of $\mathrm{V} 13$ in the 1958-2012 period. The pink and gray strips indicate the positions of the fundamental mode and its first harmonic detected at the different epochs.

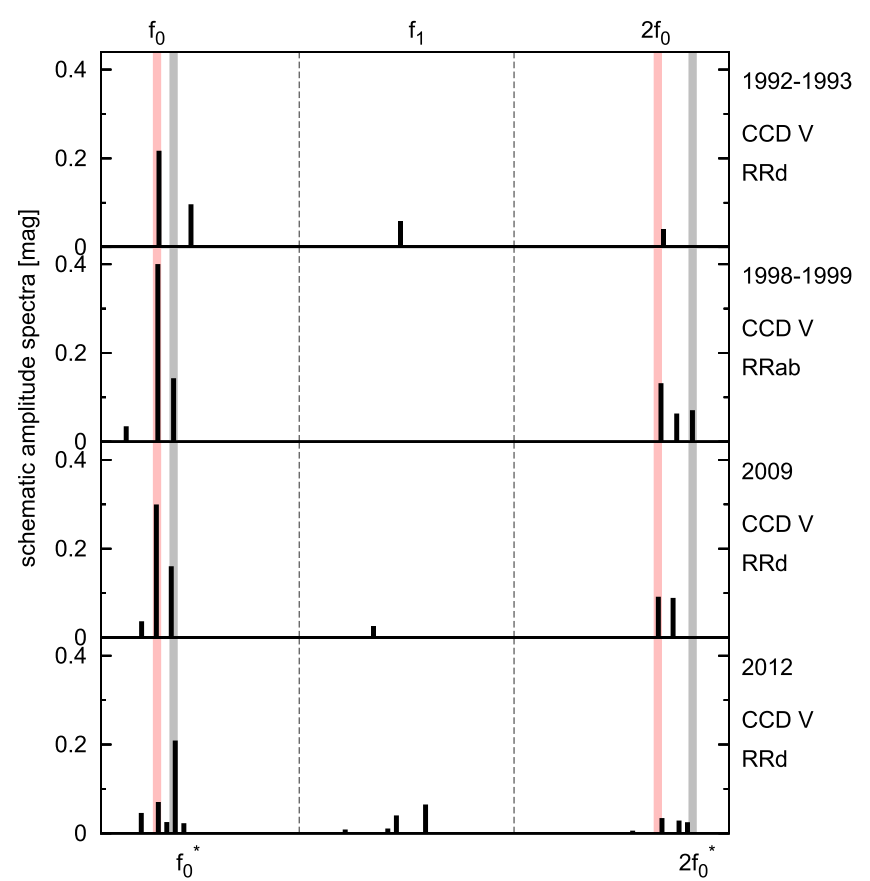

Figure 18. The same as Figure 13 for the frequency components of V44 in the 1998-2012 period.

models still fail to explain the anomalous period ratios observed in M3 and in the Galactic Bulge (C04, Smolec et al. 2015a), and V13 is one of the RRd stars with a too small period ratio. According to D12 and the combined K98, B06, and $\mathrm{J} 12$ data, its $f_{1 \mathrm{O}} / f_{\mathrm{F}}=0.7315$ frequency ratio is even smaller than that derived by $\mathrm{C} 04$ (0.7379).

V13 was identified first as a double-mode variable by C04. It had been classified as an RRab star in all previous studies. The 
fundamental mode was the dominant mode in each available data set.

The pulsation period decreased drastically by 0.0036 days $\left(\Delta f_{\mathrm{F}}=0.015 \mathrm{day}^{-1}\right)$ in the $1960 \mathrm{~s}$. The period change was accompanied by a $25 \%$ decrease of the fundamental-mode amplitude. Unfortunately, the quality of the photographic data does not make it possible to detect small-amplitude signals in these data. Therefore, it cannot be excluded that the strong period change and amplitude decrease were synchronous with the appearance of the overtone mode.

The reanalysis of all the CCD data shows that, in fact, the overtone mode is present in all observations. The 0.015 day $^{-1}$ frequency shift of the fundamental mode preceding the appearance of the 10 mode is about twice the modulation frequency $\left(0.0072 \mathrm{day}^{-1}\right)$ detected in the CCD data. The $f_{\mathrm{F}}$, $2 f_{\mathrm{F}}$, and $f_{1 \mathrm{O}}$ frequencies identified in the different data sets of V13 are shown schematically in Figure 17.

\subsection{The Double-mode Stars}

V44 was classified as an RRab star with a complex lightcurve variation assigned to a very strong Blazhko effect previously. Its 0.506 day fundamental-mode period is significantly longer than the $0.453-0.485$ day period of the other RRd stars. There are 18 RRab stars in M3 that do not show the overtone mode, but their periods are shorter than the F-mode period of V44.

We find, however, that frequency around the possible position of the $1 \mathrm{O}$ mode also appears in the $\mathrm{C} 01$ and J12 observations, not only in the D12 data. It is the highest amplitude signal in the residual spectra of these data sets, but $1 \mathrm{O}$ is not detected in the H05 and B06 observations. The amplitude of the fundamental mode is 1.5-2.0 times larger in the H05 and B06 data than in those observations when the overtone mode is also present, although with a small amplitude.

The detected frequencies of V44 at the positions of $f_{\mathrm{F}}, 2 f_{\mathrm{F}}$, and $f_{10}$ are shown schematically for the available CCD data sets in Figure 18. The frequency of the main component at $f_{\mathrm{F}}$ is 0.011 day $^{-1}$ larger (the period is 0.0029 days shorter) in the D12 data than it was previously. The frequency, which is identified as $f_{\mathrm{F}}-f_{\mathrm{m}}$ in the D12 data, is the largest amplitude signal in the 2009 observations and in all other CCD data. Despite the fundamental-mode frequency having a significantly larger amplitude than the largest side frequency (the amplitude ratio is $\sim 3$ ) in the D12 data, $2 f_{0}$ and $3 f_{0}$ are not detected; only modulation components appear in the second and third harmonic orders. In contrast, in other CCD data harmonic components of the F-mode frequency are detected up to harmonic orders $4-5$.

The frequency ratio of the radial modes has an anomalously low value in each data showing double-mode pulsation.

V68 All the CCD and photographic data yield a very similar solution with the $0.7439-0.7440$ frequency ratio and a dominant overtone mode.

V79 The unique behavior of V79 was discussed in several previous papers (e.g., Clement et al. 1997; Clement \& Goranskij 1999; Clement \& Thompson 2007; Goranskij et al. 2010). Data published in Clement \& Goranskij (1999) and Goranskij \& Barsukova (2007) are utilized in the analysis, in addition to the other CCD and photographic data.

V79 was a double-mode star only in the 1992-2007 interval. Before and after this double-mode episode it had/has been an RRab star. A strong Blazhko effect has been detected since

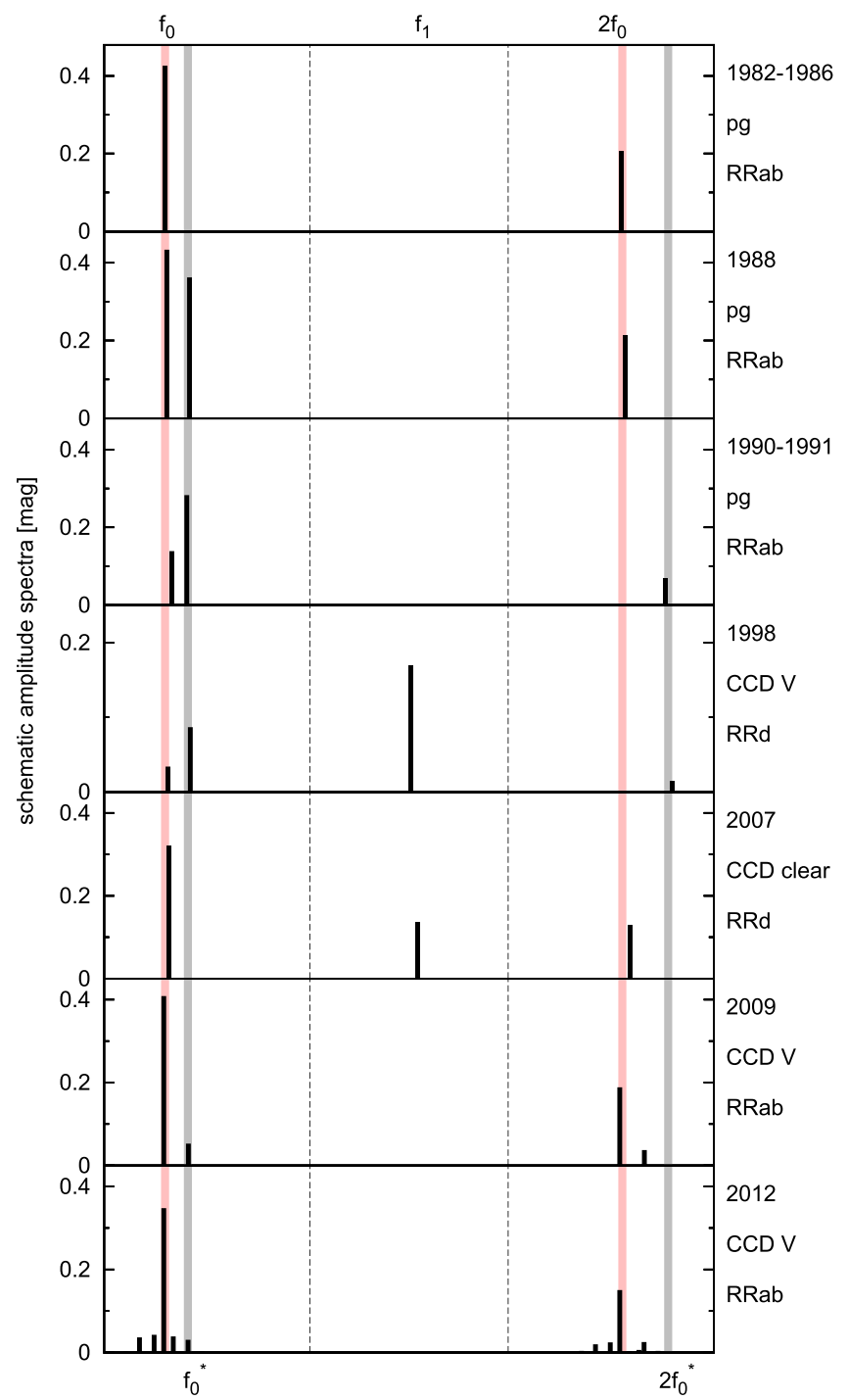

Figure 19. The same as Figure 17 for the frequency components of V79 in the 1982-2012 period. Only those epochs that give a substantially different solution are shown.

2008. Indications of Blazhko modulation of the fundamental mode are evident in many parts of the photographic data, but no Blazhko period can be derived. For completeness, we also include a summary of the peculiar changes of V79.

During the major part of its double-mode stage and just preceding it (between 1990 and 1998) the fundamental mode frequency was $0.016 \mathrm{day}^{-1}$ larger (and the period was 0.004 days shorter) than it was prior and after the doublemode episode. The amplitude of the overtone mode was about twice the amplitude of the fundamental mode in 1992-1998; the fundamental mode became the dominant mode again from 2006. The frequency ratio of the radial modes was larger in 1992-1998 and smaller than normal in 2006-2007 by about 0.002 .

The changes in the frequency spectrum of V79 is documented in Figure 19. Only the substantially different solutions are shown. Preceding the emergence of the overtone mode, the fundamental mode frequency had been split into two components and the amplitudes of the components had been transposed. In addition to the dominant frequencies observed at 2.0711 and $2.0843 \mathrm{day}^{-1}$, residual signals appeared at 2.087 
and 2.0743 day $^{-1}$ frequencies in the 1988 and 1990-1991 photographic data, respectively. The combined 1998 CCD data (H05, B06, and CG99) showed the dominant fundamental frequency at $2.0865 \mathrm{day}^{-1}$, but a frequency component at 2.0719 day $^{-1}$ also appeared in this data set. The frequency changes of the first harmonic frequency of $f_{\mathrm{F}}$ followed the displacement of the fundamental frequency. After the doublemode episode, several $k f_{\mathrm{F}}+f_{\mathrm{m}}\left(f_{0}=2.069, f_{\mathrm{m}}=0.016\right.$ day $^{-1}$ ) components appeared in the 2009 data. The frequency of the strongest modulation component was equivalent to the fundamental-mode frequency $\left(2.085 \mathrm{day}^{-1}\right)$ observed during the double-mode phase. Later, the modulation become more complex, multiple periodic with 0.006 and 0.016 day $^{-1}$ frequencies of the primary and secondary modulations. The $2.085 \mathrm{day}^{-1}$ frequency component also appears in the D12 data. However, it is only the fourth largest modulation component with 0.031 mag amplitude.

Table 9 lists the fundamental-mode period and the magnitude- and intensity-averaged mean $B, V, I_{\mathrm{C}}$ magnitudes of V79 derived from the CCD data. According to the pulsation equation (given, e.g., by Marconi \& Degl'Innocenti 2007) a 0.0043 increase of $\log L$ (i.e., a $0.011 \mathrm{mag}$ decrease in magnitude) accounts for a 0.004 day increase of the pulsation period. Although the changes of the intensity-averaged mean magnitudes may support that luminosity changes are standing behind the period increase of V79, we have to note also that differences of the order of $0.01 \mathrm{mag}$ in photometric results of GCs are common, simply because of calibration and/or instrumental effects. Moreover, the much larger decrease of the magnitude-averaged brightness may be a consequence of the sinusoidal overtone mode being dominant in 1992-1998, but the large-amplitude, asymmetric RRab-type light curve observed in 2009 and 2012 is strongly nonlinear. Consequently, the observed increase of the intensity-averaged mean magnitudes may also arise, at least partly, from the differences in the light-curve shapes.

V87 All the CCD and photographic data yield a very similar solution with a dominant overtone mode. Period changes of both modes are marginal.

V99 The period ratio of the radial modes are anomalously large according to each observation that shows double-mode behavior. The reanalysis of the photographic data has revealed that aside from the overtone mode, the fundamental mode is also detectable in the majority of the data. Most probably, the improper phase coverage of the very-long-period Blazhko cycle of the fundamental mode (Section 6.1) explains the previously detected changes of the fundamental-mode frequency. The $\sim 0.001$ day $^{-1}$ decrease of the overtone-mode frequency is probably real. Although the frequency of the $f_{\mathrm{F}}+f_{\mathrm{m}}$ component detected in the D12 data is very close to the exact 3:4 resonance position to the overtone mode $\left(\left(f_{\mathrm{F}}+f_{\mathrm{m}}\right) / f_{1}=0.7498\right)$, the amplitude of this modulation component is only half of the amplitude of $f_{\mathrm{F}}-f_{\mathrm{m}}$. Even in such a close-resonance position, there is no sign that the 3:4 resonance would be in action (see also in Jurcsik et al. 2014).

V125 The fundamental mode is detected only in the D12 data. Its very small amplitude makes the detection of this component in the previous data unlikely. The period of the overtone mode is very stable. The star is not over-luminous and lies at the blue edge of the double-mode region.
Table 9

Period and Magnitude Changes of V79 between 1992 and 2012

\begin{tabular}{lcccccccc}
\hline \hline Year & \multirow{2}{*}{$P_{0}$} & $\langle B\rangle_{\mathrm{i}}$ & $\langle B\rangle_{\mathrm{m}}$ & $\langle V\rangle_{\mathrm{i}}$ & $(V)_{\mathrm{m}}$ & $\left\langle I_{\mathrm{C}}\right\rangle_{\mathrm{i}}$ & $\left\langle I_{\mathrm{C}}\right\rangle_{\mathrm{m}}$ & Data \\
\cline { 3 - 9 } & & $(\mathrm{mag})$ & $(\mathrm{mag})$ & $(\mathrm{mag})$ & $(\mathrm{mag})$ & $(\mathrm{mag})$ & $(\mathrm{mag})$ & \\
\hline $1992-1993$ & 0.47948 & 15.95 & 15.94 & 15.67 & 15.66 & $\ldots$ & $\ldots$ & $\mathrm{C} 01$ \\
1996 & 0.4798 & $\ldots$ & $\ldots$ & 15.71 & 15.70 & $\ldots$ & $\ldots$ & $\mathrm{K} 98$ \\
1998 & 0.47927 & 16.00 & 15.99 & 15.72 & 15.71 & 15.28 & 15.28 & $\mathrm{H} 05, \mathrm{~B} 06$ \\
1998 & 0.47927 & $\ldots$ & $\ldots$ & 15.71 & 15.70 & $\ldots$ & $\ldots$ & $\mathrm{CG}(1)$ \\
2009 & 0.48329 & $\ldots$ & $\ldots$ & 15.78 & 15.72 & $\ldots$ & $\ldots$ & $\mathrm{J} 12$ \\
2012 & 0.48329 & 16.06 & 16.00 & 15.76 & 15.72 & 15.31 & 15.29 & $\mathrm{D} 12$ \\
\hline
\end{tabular}

Notes. The subscripts " $\mathrm{m}$ " and "i" refer to magnitude- and intensity-averaged mean brightness values.

Reference. (1) Clement \& Goranskij (1999).

V166 Double-mode behavior is observed in each CCD data set. Both the fundamental- and the overtone-mode light curves are modulated. The period ratio is anomalously small.

V200 Each CCD data show double-mode behavior. The $\mathrm{H} 05$, B06, and the D12 data yield a similar light-curve solution with similar amplitudes of the fundamental and the overtone modes and with a normal period ratio. The $\mathrm{C} 01$ observations of V200 are not publicly available. Based on the analysis of a reprocessed version of the C01 data, C04 listed V200 as one of the double-mode stars with a too small period ratio. However, the strong aliasing of the $\mathrm{C} 01$ data makes the frequency determinations of $\mathrm{C} 04$ dubious. The strongest alias frequencies appearing in the $\mathrm{C} 01$ observations are separated by 0.003 , 0.006 , and $0.009 \mathrm{day}^{-1}$. The differences between the $f_{\mathrm{F}}$ and $f_{1 \mathrm{O}}$ frequencies given by $\mathrm{C} 04$ and found in the other observations are -0.006 and $0.009 \mathrm{day}^{-1}$, which supports that, probably, no significant period change occurs in this star. No difference between the amplitude ratios of the fundamental and firstovertone modes larger than expected from the uncertainties is seen between the solutions of the H05, B06, and the D12 data. The amplitude changes of the modes found by $\mathrm{C} 04$ between the 1992 and 1993+1997 seasons are probably the consequence of the inadequacy of the data sampling and the mistaken frequency identification. Based on the characteristics of its light curve and period ratio, this star seems to be the twin of V68.

V251 The frequencies published by $\mathrm{C} 04$ and B06 are significantly different from the results obtained from the D12 data. The reprocessed C01 data of V251 analyzed in C04 are not publicly available. The differences between the fundamental- and overtone-mode frequencies given by $\mathrm{C} 04$ and found in the present analysis are 0.009 and $-0.003 \mathrm{day}^{-1}$. Thus, it seems that aliasing might have also biased the frequency determination of V251 in C04. The frequencies of V251 given by B06 are $0.03-0.04$ day $^{-1}$ larger than those detected in the D12 data. Although the frequencies identified by B06 are indeed the largest amplitude signals in the Fourier spectrum of the data, a solution with frequency values close to the results of the D12 data fits the observations with $7 \%$ smaller residual scatter. C04 reported significant amplitude changes of the modes, however, the D12 data do not show any frequency in the residual spectrum at the radial modes, indicating that the light curves of the modes are stable.

V252 The C01 observations of V252 are not publicly available. The combined $\mathrm{H} 05$ and B06 data yield a similar solution as the D12 data. 
Non-Blazhko RRd stars
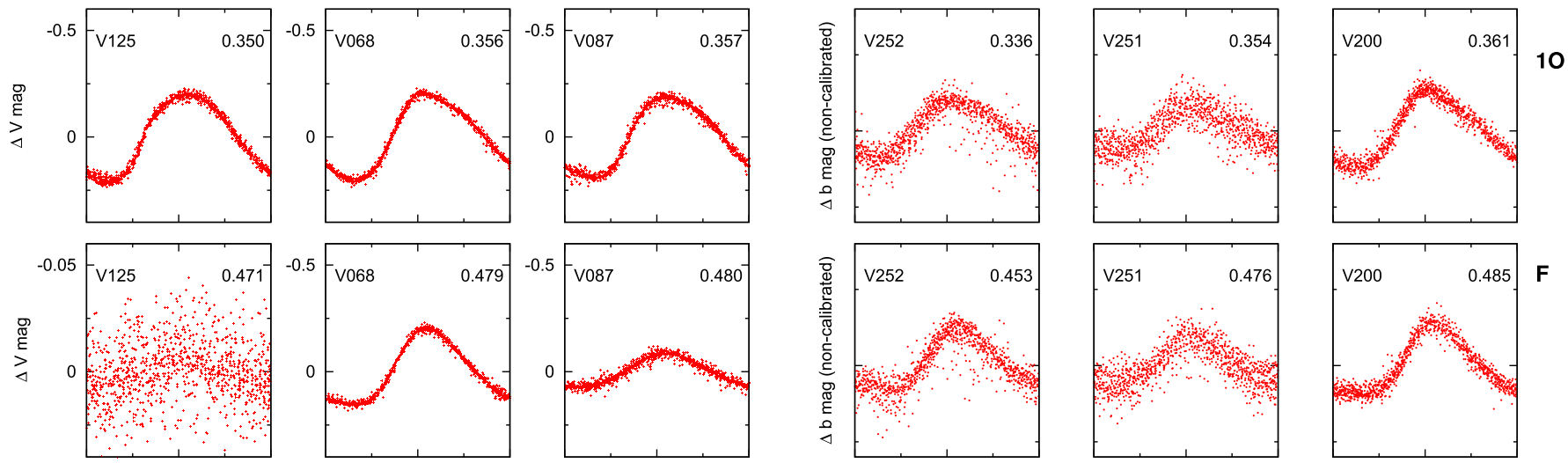

\section{Blazhko RRd stars}
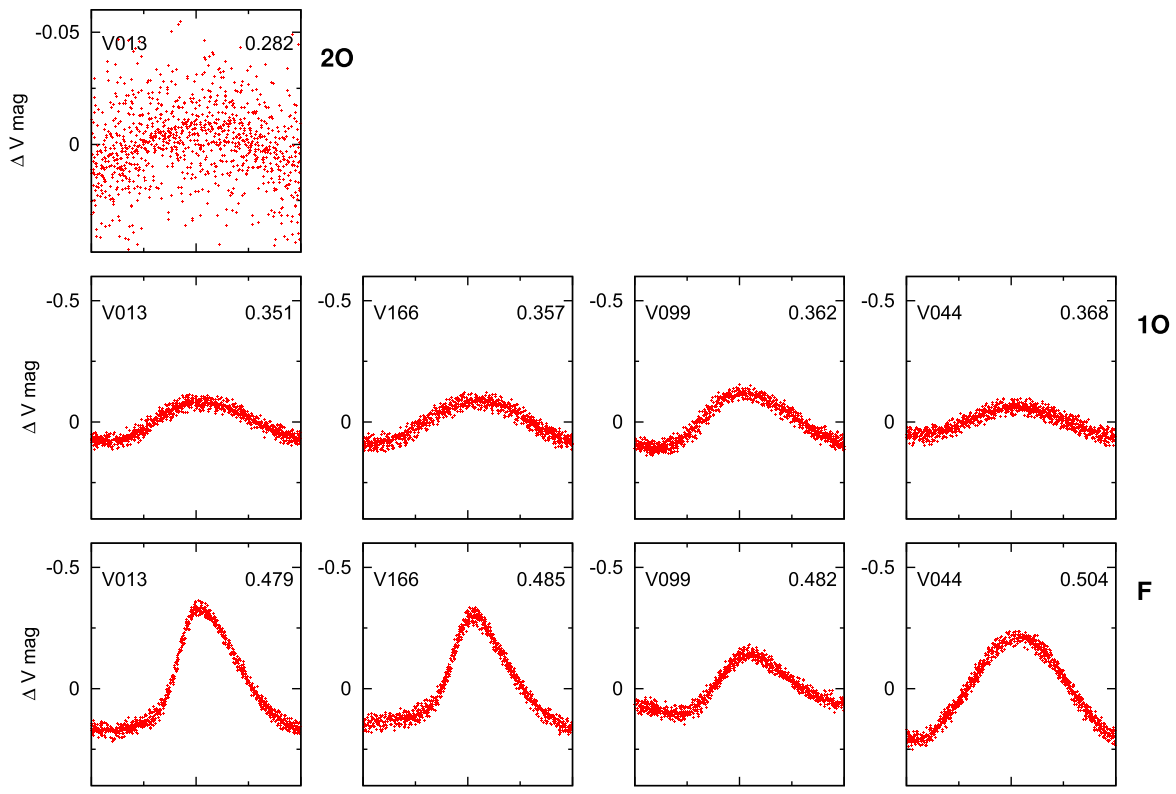

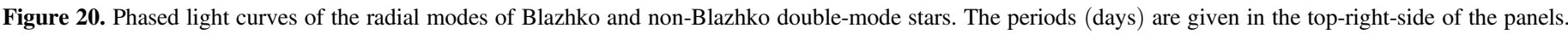
The data are prewhitened for all the other signals.
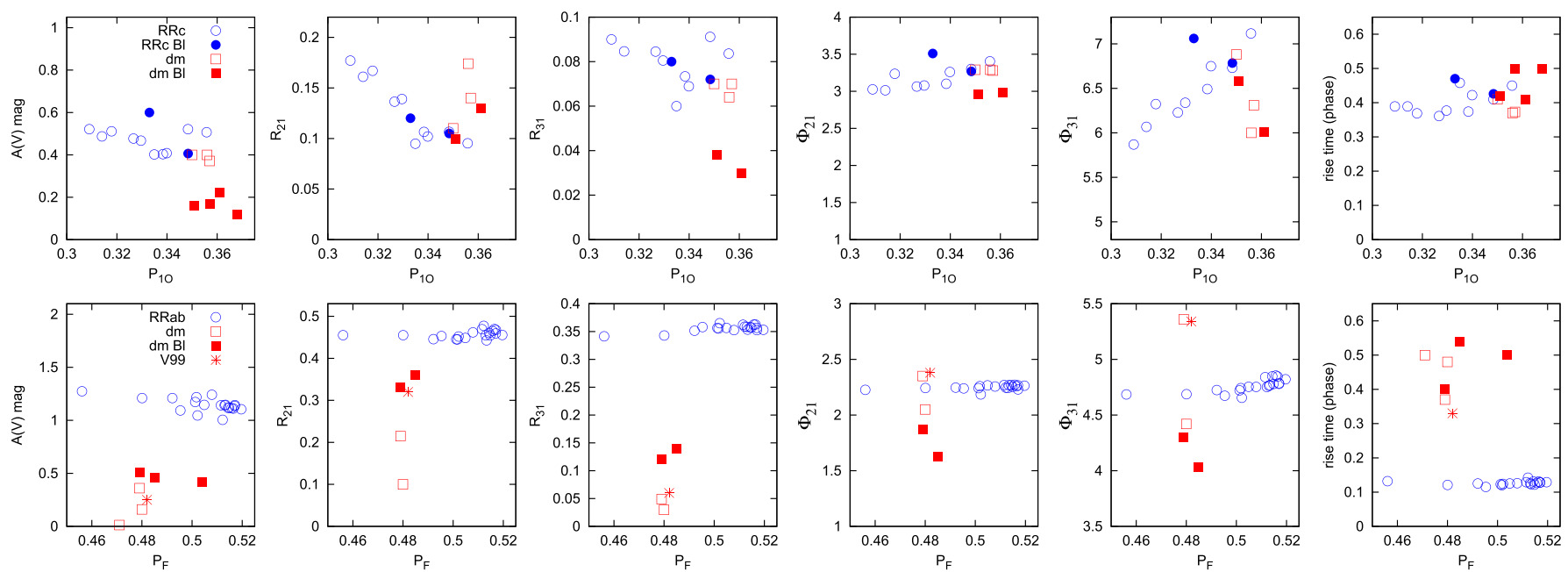

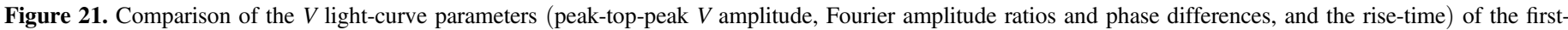

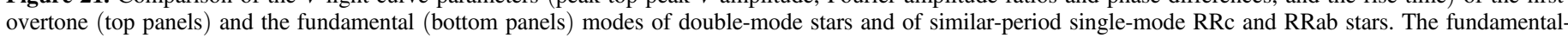

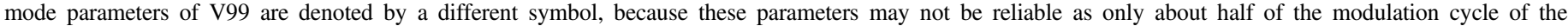
fundamental mode is covered by the observations. 
Our analysis shows that in normal period ratio RRd stars of M3 (V68, V87, V125, V200, V251, and V252) either the overtone mode is dominant or the fundamental mode has a similar amplitude as the overtone. The majority of the known RRd stars share this property. Based on the results given in Table 8, the modal content (i.e., the frequencies) and the amplitude- and period-ratios of V68 and V87 have been stable during the century covered by the observations. Most probably, $\mathrm{V} 125$, at the blue border of the double-mode region, is in the stage of the fall-off of the weak fundamental-mode component. This is supported by the similarity of the periods, amplitudes, colors, and the light curves' shapes of V125 and V126 (the longest period not over-luminous RRc star in M3). We suppose that the differences between the amplitude ratios and the frequencies published in $\mathrm{C} 01, \mathrm{C} 04, \mathrm{H} 05$, and $\mathrm{B} 06$, and obtained from the D12 data for V200, V251, and V252 do not reflect real changes of the double-mode properties of these stars, but arise from the strong aliasing problems of the previous data, which may have biased the previous frequency determinations. We conclude that the pulsation properties of double-mode stars with normal period ratio are stable; they do not change significantly on a timescale of about a century in $\mathrm{M} 3$.

\section{DISCUSSION ON THE PROPERTIES OF BLAZHKO RRD STARS}

RR Lyrae stars showing Blazhko effect were first reported by Soszyński et al. (2014). A detailed analysis of these special variables found in the OGLE Galactic bulge collection was published recently by Smolec et al. (2015a). It is also probable that long-period Blazhko modulation accounts for the detected changes of the amplitude ratios of the radial modes of NSVS 5222076 (Hurdis \& Krajci 2012). The Blazhko modulation of 1O/2O double-mode Cepheids were discussed in detail by Moskalik \& Kołaczkowski (2009).

Modulation is observed among 1O/2O LMC Cepheids either in both modes or neither of them (Moskalik \& Kołaczkowski 2009). The same is true for F/1O double-mode RR Lyrae stars in M3. However, despite the large-amplitude modulations of the $\mathrm{F}$ and $1 \mathrm{O}$ modes, the small-amplitude $2 \mathrm{O}$ mode of $\mathrm{V} 13$ is not modulated.

Moskalik \& Kołaczkowski (2009) explained the anti-phase modulations of the radial modes of Cepheids by non-stationary resonant coupling of one radial mode with the another radial or a non-radial mode, and with the sharing on the excitation sources between the two radial modes. Resonances are in fact detected in Cepheids (e.g., a 2:1 resonance produces the wellseen features of the light curve of bump-Cepheids). Therefore, we checked whether the light curves of the radial modes of the Blazhko double-mode stars show any systematic difference from the light curves of other double-mode stars and from single-mode RR Lyrae stars. Figure 20 shows the light curves of the radial modes of double-mode stars. The data were prewhitened for the Fourier components of the other mode, for the modulation of both modes, and for the linear-combination terms. The only conspicuous difference between the light curves of the Blazhko and non-Blazhko double-mode stars is that the overtone mode is the dominant mode (or the modes have similar amplitudes: V200, V251, V252) in RRd stars without light-curve modulation, whereas the fundamental mode has a larger amplitude than the overtone in Blazhko RRd stars.

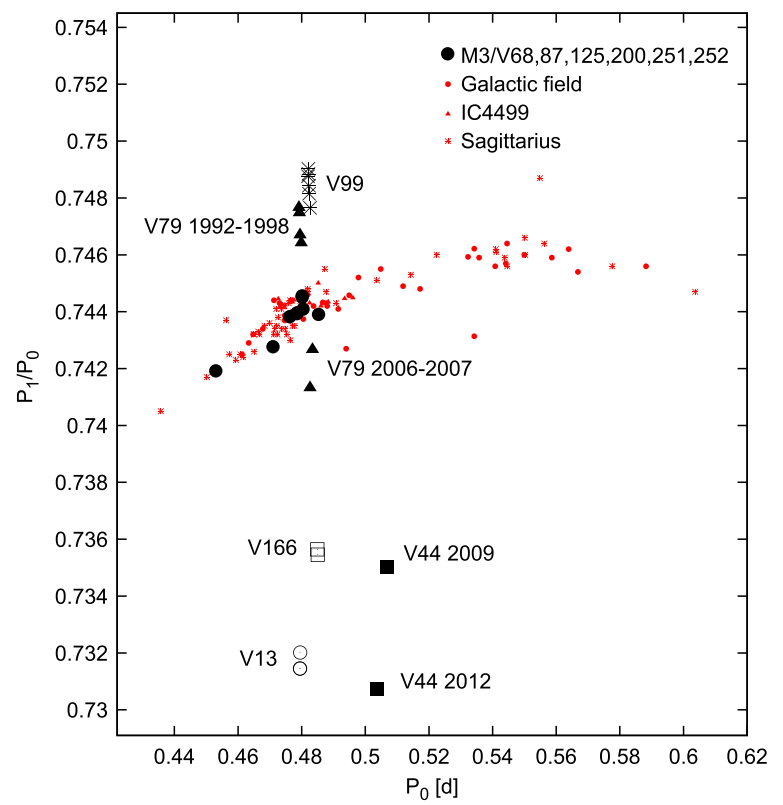

Figure 22. Petersen diagram, $P_{1} / P_{0}$ period ratio vs. $P_{0}$, of the M3 double-mode stars is shown. Filled circles denote RRd stars with a normal period ratio (V68, V87, V125, V200, V251, and V252). Other symbols denote the results obtained for Blazhko RRd stars at different epochs according to the data given in Table 8. For comparison, the period ratios of double-mode stars in the Galactic field, IC 4499, and the Sagittarius dwarf galaxy are also shown.

There is no easily seen distortion in the mean light curves of the radial modes of Blazhko stars (see in Figure 20). The fundamental-mode light curves of both Blazhko and nonBlazhko RRd stars differ from the light curves of single-mode RRab stars systematically; however, the lack of the higherorder harmonic components in RRd stars may mostly explain the differences.

None of the light curves at the different Blazhko phases shown in Figure 15 indicate that any resonance would affect their shapes. Amplitude and phase modulations of both modes of the Blazhko double-mode stars are evident; however, the light curve is smooth in each Blazhko phase. Only the fundamental mode of V44 displays irregularities, but this is probably produced by the complexity of the modulation.

The $V$ light-curve parameters of the fundamental and firstovertone modes of double-mode stars and of similar-period single-mode stars are shown in Figure 21. The period; peak-topeak $V$ amplitude; $R_{21}, R_{31}, \varphi_{21}, \varphi_{31}$ Fourier amplitude ratios and phase-differences; and rise-time (in phase units) are plotted versus the overtone- and fundamental-mode periods in the panels. The Fourier amplitude ratios and phase differences are not shown for the most sinusoidal components of the doublemode stars. The only systematic difference between the lightcurve parameters of the overtone mode of Blazhko and nonBlazhko RRd stars is that the $A$ amplitudes and the $R_{31}$ amplitude ratios, and maybe the $\varphi_{21}$ phase differences of Blazhko RRd stars, are systematically smaller than those of the normal RRd and RRc stars. The rise-time of each RRd star is around the same as the rise-time of RRc stars (between 0.4 and $0.5)$. The amplitudes and amplitude ratios of the fundamental mode of both Blazhko and non-Blazhko RRd stars are significantly smaller than those in normal RRab stars (the amplitude ratios of normal RRd stars are even smaller than of Blazhko RRd stars); the phase differences of Blazhko RRd 

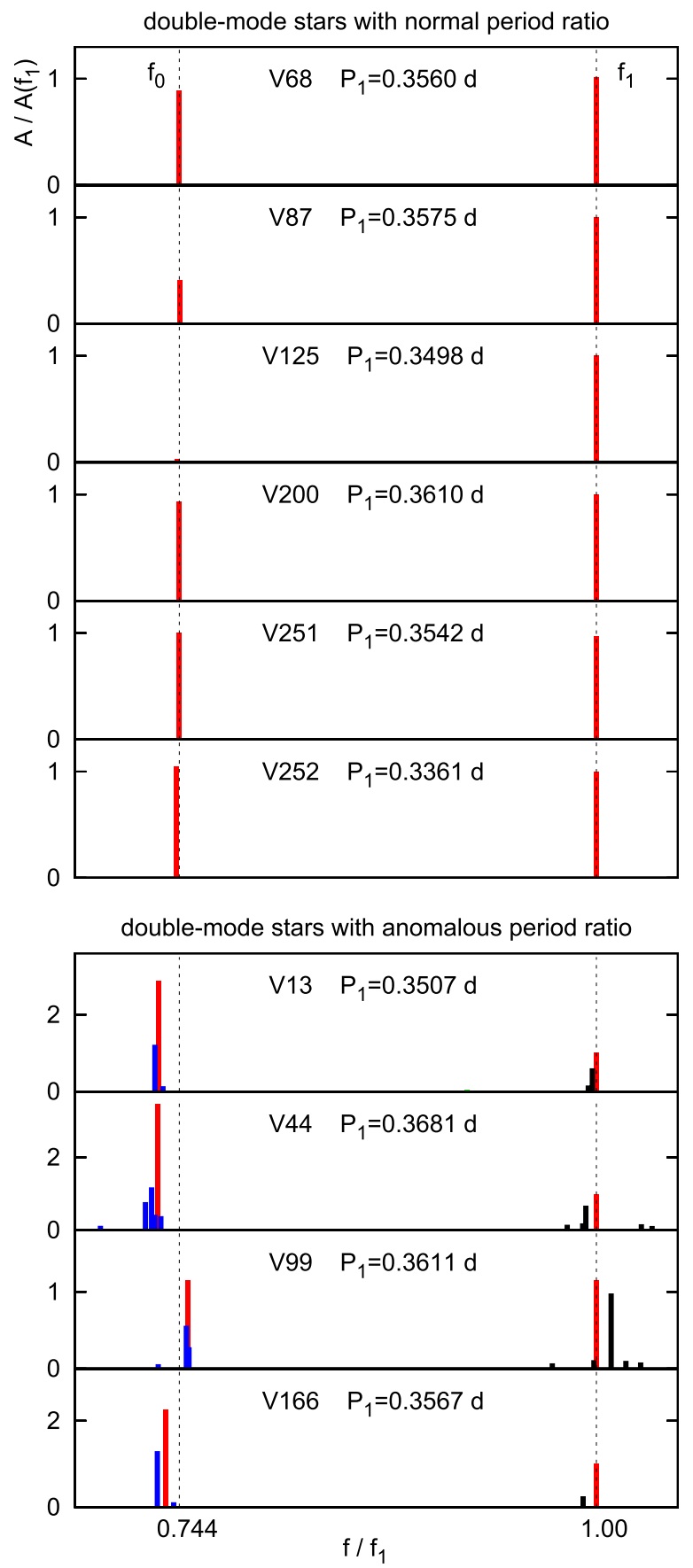

Figure 23. The fundamental- and overtone-mode frequencies of RRd stars in M3. The radial-mode frequencies of RRd stars with a normal period ratio $\left(P_{1} / P_{0}=0.742-0.745\right)$ are separate, single signals. By contrast, complex multiplets at the radial-mode frequencies, characteristics of Blazhko modulation, are detected in RRd stars, which have anomalous period ratios.

stars are also too small, whereas the phase differences of normal RRd stars seem to be scattered around the values of RRab stars. The rise time of the fundamental mode of each $\mathrm{RRd}$ star is significantly larger than the rise time of RRab stars.

Based on the light curve's shapes and the Fourier parameters of the Blazhko RRd stars, we have not found any clear evidence of a distortion caused by resonance interaction.

In Jurcsik et al. (2014) we have shown that the anomalous period ratio is a common property of RRd stars showing Blazhko modulation of the radial modes in M3. The opposite is also true, none of the RRd stars with a normal period ratio shows modulation of any of the modes. A similar tendency has also been detected in the Galactic bulge data (Smolec et al. 2015a). The anomalous period ratios of RRd stars cannot be interpreted by any canonical explanation as was discussed in detail in C04 and Smolec et al. (2015a).

In Figure 22, the $P_{1 \mathrm{O}} / P_{\mathrm{F}}$ period ratio of $\mathrm{M} 3$ double-mode stars is shown in comparison with results of the double-mode stars in the Galactic field, the IC 4499 GC, and the Sagittarius dwarf galaxy (compilation by Walker \& Nemec 1996; Cseresnjes 2001; McClusky 2008; Wils 2010). The period ratios of six RRd stars (V68, V87, V125, V200, V251, and V252) fit the region covered by the data of the other systems. The period ratios of Blazhko RRd stars (V13, V44, V79, V99, and V166) are anomalous according to each of the data, which show double-mode behavior (see Table 8).

The frequencies appearing at the positions of $f_{\mathrm{F}}$ and $f_{1 \mathrm{O}}$ of RRd stars in the D12 data are shown in Figure 23. Contrary to the single signals of the radial modes of normal period-ratio RRd stars, the frequency spectrum of Blazhko RRd stars are comprised from multiplets at the fundamental- and the overtone-mode frequencies. The highest-amplitude frequency belongs to the fundamental mode in these stars.

The data given in Table 8 and Figures 17-19 indicate that in Blazhko RRd stars not only are the period ratios anomalous, but from time to time, a different component of the multiplets is the dominant component. OGLE-BLG-RRLYR-02530 (Smolec et al. 2015a) seems to be a similar case. We have also checked whether any pair of the components of the multiplets would yield a period ratio in the required $0.743-0.744$ range. However, the mystery of the anomalous period ratios of Blazhko RRd stars cannot be resolved in this way; none of the components of the multiplets can give a proper period ratio.

Connected to the switch between the single- and doublemode states (just preceding or following it), jumps of the fundamental-mode frequency as large as $0.010-0.015 \mathrm{day}^{-1}$ (0.003-0.004 days jumps in the periods) are detected in the Blazhko RRd stars V13, V44, and V79. The frequency shift preceding the switch to the double-mode stage is always positive (the period is decreasing) and it is accompanied by a strong amplitude decrease of the fundamental mode in all these stars.

Blazhko RRab stars are known to show large, irregular, rapid period changes (J12); however, all these changes are at least about an order of a magnitude smaller than the period changes observed in Blazhko double-mode stars. The switch from double-mode to fundamental-mode pulsation of OGLE-BLGRRLYR-12245 (Soszyński et al. 2014) was accompanied by a 0.0005 day period increase $\left(-0.003\right.$ day $^{-1}$ in frequency). The period change was attributed to a nonlinear shift caused by the four-times increase of the amplitude of the fundamental mode by Soszyński et al. (2014). Although simultaneously with the appearance of the overtone mode, amplitude decrease of the fundamental mode of V13, V44, and V79 is also detected, because their period shifts are about an order of a magnitude larger than in OGLE-BLG-RRLYR-12245; therefore, nonlinearity cannot explain the observed frequency shifts in these stars completely.

The displacement of the frequencies corresponds to the modulation frequency (half of it in V13) indicating that, instead of real frequency changes, the modulation is so strong that the side frequency has the largest amplitude in these cases. 

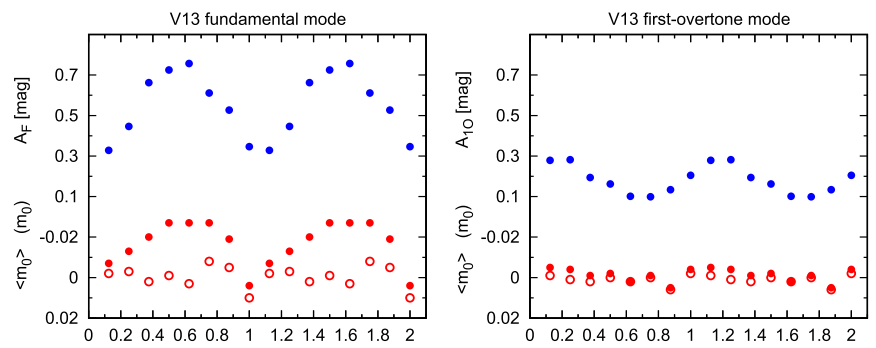

V166 fundamental mode

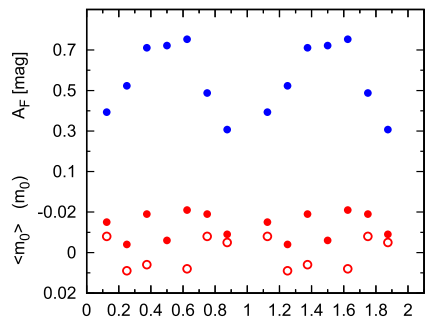

Blazhko phase

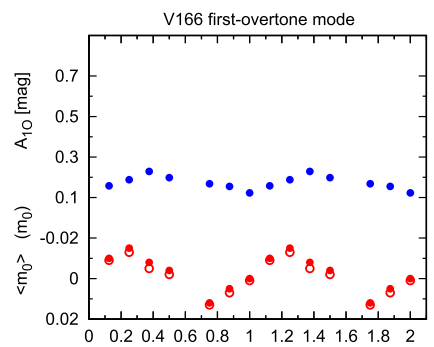

Blazhko phase

Figure 24. Variations of the fundamental- (left-side panels) and overtonemode (right-side panels) amplitudes and the magnitude- and intensity-averaged mean $V$ magnitudes $\left(\left\langle m_{0}\right\rangle\right.$ and $\left(m_{0}\right)$ shown by open and filled circles, respectively) of the light curves of V13 (top panels) and V166 (bottom panels) during the Blazhko cycle. Note that the Blazhko periods of the fundamental and overtone modes are the same for V13, but they are 71.5 and 43.5 days, respectively, for V166. The results are derived for the light curves in the eight Blazhko phases shown in Figure 15. The light curves are prewhitened for the pulsation and modulation of the other radial mode and for the linearcombination terms.

The dominant component is the $f+f_{\mathrm{m}}$ frequency in the double-mode stage of these three stars. Thus, the anomalously low period ratios would become even lower if the pre-RRd frequency of the fundamental-mode were taken as the real fundamental-mode frequency in V13 and V44.

Anomalous period ratios are detected mostly (probably exclusively) in Blazhko RRd stars. Assuming that pulsation models give the correct results for the period ratios of RR Lyrae stars (as in the case for non-Blazhko RRd stars), we have to think that the stellar structure of Blazhko and non-Blazhko stars should be different in order to explain the anomalous period ratios of Blazhko RRd stars.

The period ratios of the modulations of the overtone and the fundamental modes $\left(P \mathrm{~m}_{1 \mathrm{O}} / P \mathrm{~m}_{\mathrm{F}}\right)$ of the double-mode Blazhko stars (V13, V44, V99, and V166) match the 1:1, 4:7, 1:10, and 3:5 small-integer ratios within about the error limits of the periods. The modulation-period ratios of some of the Galactic bulge Blazhko RRd stars are also close to small-integer ratios (e.g., it is close to 3:10, 2:3, 1:3, 4:7, 1:5, 2:3 in OGLE-BLGRRLYR-04598, -07393, -10728, -11311, -22356, and -32462, respectively).

It was already shown that the light curves of some Blazhko RRab stars were modulated with different periods either simultaneously or at different epochs, and that the ratios of the modulation periods of multiple-periodic Blazhko stars were close to the ratios of small-integer numbers (Jurcsik et al. 2011, 2012b; Sódor et al. 2011; Benkő et al. 2014). In other stars, different components of the multiplets reflect the dominant modulation at different epochs (i.e., the amplitudes of the multiplet components vary in time), according to the long-term data as, for example, in V44, V140/M3 (this paper), and V2/ M5 (Jurcsik et al. 2011). In Jurcsik et al. (2011), we wrote "If these modulations correspond to elements of series of equidistant spacing multiplets, their frequency ratios are close
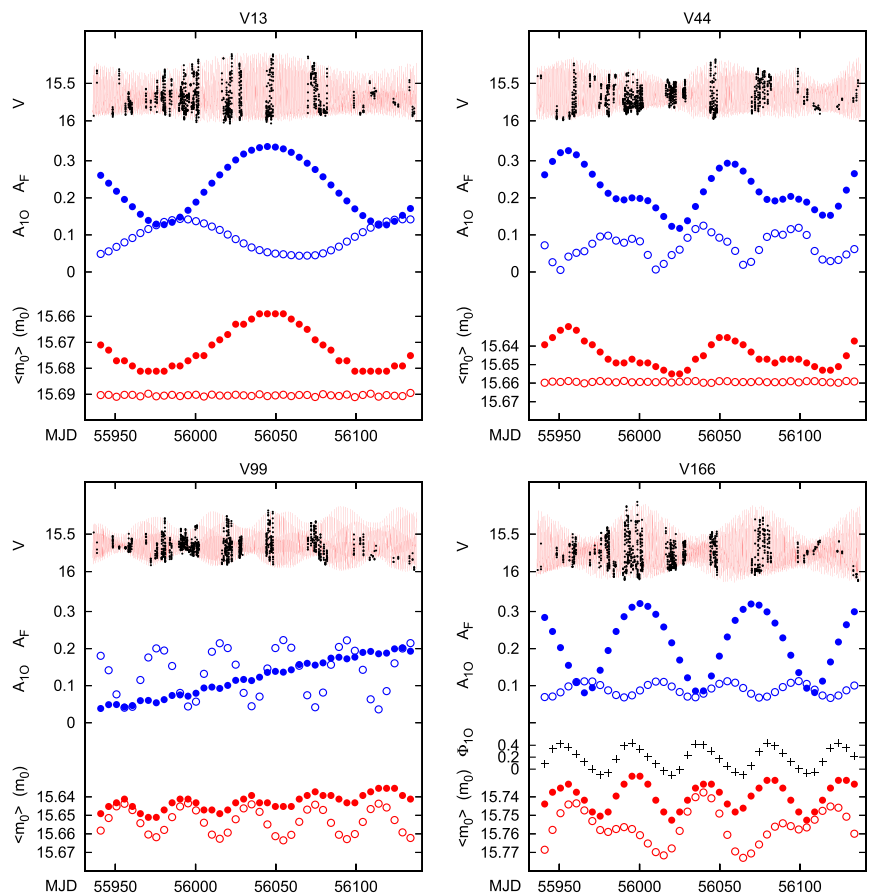

Figure 25. Amplitudes $\left(A_{10}\right.$ and $A_{\mathrm{F}}$ shown by open and filled blue circles, respectively) and magnitude- and intensity-averaged mean brightness (shown by open and filled red circles, respectively) of Blazhko RRd stars derived from 5 day segments of the synthetic data generated from the full light-curve solutions. In the top part of each plot the synthetic light curves are shown with the observations over-plotted. The phase variation of the overtone mode of V166 is also shown by "+" symbols.

to fractions of small-integer numbers. This indicates that the observed modulation frequency values may not always correspond to the 'base frequency,' (i.e., the shortest frequency component of a multiplet)." The ratios of the modulation periods of the $\mathrm{F}$ and $1 \mathrm{O}$ modes in Blazhko double-mode stars may be explained similarly. If both modes select their modulation frequency from the same rich multiplet spectrum, thean any small-integer ratio of the modulation periods is equally possible.

Smolec et al. (2015a) found a "feedback effect" between the star's brightness and pulsation-mode amplitude in some of the OGLE Blazhko RRd stars. They noticed that the "larger the amplitude of the dominant mode, smaller the mean stellar brightness."

The connection between the mean brightness and amplitude variations is checked both on the observed and on synthetic data. The magnitude- and intensity-mean $V$ brightnesses and the amplitudes of the $\mathrm{F}$ and 10 modes are shown in Figure 24 as derived from the disentangled light curves of the radial modes of V13 and V44: the two Blazhko RRd stars that have good pulsation-phase coverage of both radial modes in most of the Blazhko phases. The same parameters are also determined by fitting the radial-mode frequencies and their linear combinations to 5 day segments of synthetic data of the four Blazhko RRd stars (Figure 25). The synthetic data are generated from the modulated radial modes' components of the full light-curve solutions.

Mean-brightness variations are indeed detected in each of the four Blazhko RRd stars. The intensity-averaged mean brightness differs significantly from the magnitude-averaged value in some cases, because of the non-sinusoidal shape of the light curves, which is especially true for the fundamental mode in 
the large-amplitude phase of the modulation. If we accept that the intensity-averaged mean-brightness variation reflects the luminosity changes-as was shown in Blazhko stars (e.g., in Jurcsik et al. 2008, 2012b; Sódor et al. 2011) using the photometric Baade-Wesselink method (Sódor et al. 2009)_the brightness variations of V13 and V44 are in phase with the amplitude variations of the dominant fundamental mode. The intensity-averaged mean-brightness variation of V99 is twofold -it varies in anti-phase with the amplitude changes of the overtone mode, and a gradual brightness increase, parallel with the amplitude increase of the fundamental mode, is also observed. The amplitude changes of V166 are the most surprising: the intensity-averaged mean brightness does not show any systematic changes with the modulation period of the dominant fundamental mode, which shows large-amplitude modulation; instead, it varies with the modulation period of the overtone mode. The brightness variation is in a $\pi / 2$ phase lag with the very small amplitude changes of the overtone mode. The intensity-averaged mean brightness follows the phase variation (also shown in Figure 25 for V166) of the overtone mode. As a summary, no universal connection between the brightness and the amplitude variations seems to exist.

\section{SUMMARY}

The light variation of overtone and double-mode RR Lyrae stars has been investigated based on an extended new $B V I_{C}$ time-series photometry and on archive data. The GC data has a unique potential to map the distribution of variables showing different properties and extra features in a large and homogeneous sample of stars.

Peculiarities of the behavior of stars showing a frequency component at 0.61 frequency ratio, the Blazhko effect, $\mathrm{PD}$, and double-mode pulsation are discussed in detail.

The most important findings of our study are summarized in the following.

1. The $f_{0.61}$ frequency is observed in $14 \mathrm{RRc}$ stars, and three normal and one anomalous period-ratio RRd stars. Section 4.

2. The amplitude and frequency of the $f_{0.61}$ component show considerable changes, whereas the radial-mode component remains stable. Section 4.1.

3. The light curves of $\mathrm{RR}_{0.61}$ stars are sinusoidal, with a reduced, if any, bump preceding maximum brightness. Section 4.2.

4. The $f_{0.61}$ frequency occurs in four RRd stars, in each RRc star at the blue side of the double-mode region, and in some bright, evolved variables with periods longer than the period of the 10 mode of RRd stars. It is detected in each RRc star with $B-V \gtrsim 0.24$ mag and $V-I \gtrsim 0.36$ mag color indices. Connection between the double-mode pulsation and the appearance of the $f_{0.61}$ frequency is proposed based on the distribution $R_{0.61}$ stars. Section 4.3.

5. PD of the small-amplitude $2 \mathrm{O}$ mode of V13 $\left(3 f_{2 \mathrm{O}} / 2\right)$ and the fundamental mode of V166 $\left(7 f_{\mathrm{F}} / 2\right)$ are detected. The Blazhko effect of both the $\mathrm{F}$ and $1 \mathrm{O}$ modes of V13 and V166 is observed, but the $2 \mathrm{O}$ mode of V13 does not show any modulation. No half-integer component of any firstovertone or $f_{0.61}$ frequency has been detected. Section 5 .

6. Blazhko modulation of both radial modes of four RRd stars and three luminous RRc stars are observed. A small- amplitude frequency component appears in the residual spectra of one normal-brightness RRc star and in a 20 variable close to the overtone-mode frequency. Strong phase modulation characterizes the modulation of the overtone modes. Section 6.

7. Two new double-mode stars are identified: F-mode frequency is detected in the RRc star, V125, and 10 mode frequency in the RRab star, V44. Section 7.

8. The most complex variability is detected in V13, which shows three radial modes, strong Blazhko effect of both the $\mathrm{F}$ and 10 modes, $\mathrm{PD}$ of the $2 \mathrm{O}$ mode, and the $f_{0.61}$ frequency component. Although modeling the radialmode frequencies of triple-mode stars may yield accurate physical parameters, unfortunately, this is not the case in V13. Pulsation models fail to explain the anomalous period ratios of some RRd stars observed in M3 and in the Galactic bulge (C04, Smolec et al. 2015a), and V13 is one of the Blazhko RRd stars with an anomalously small $P_{1 \mathrm{O}} / P_{\mathrm{F}}$ period ratio. Section 7.1 .

9. The modal content and frequencies of RRd stars seems to remain stable during the timescale of the photometric observations of M3 for variables with period ratios matching the sequence defined by the majority of the known RRd stars on the $P_{1 \mathrm{O}} / P_{\mathrm{F}}-P_{\mathrm{F}}$ Petersen diagram (V68, V97, V200, V251, and V252). Section 7.2.

10. The period ratio of four RRd stars (V13, V44, V99, and V166) are anomalous, as was the case for V79 during its double-mode stage. To explain the anomalous period ratios of Blazhko RRd stars is a great challenge for stellar pulsation theory. The anomalous period ratios of Blazhko RRd stars can, in principle, be explained by extreme highor low-metallicity models (see Figure 7. in Smolec et al. 2015b), however, it is unlikely in about $50 \%$ of the RRd stars in M3, as no extreme metallicity value for any other star in the cluster has been reported. Each anomalous period-ratio RRd star shows the Blazhko modulation of both the $\mathrm{F}$ and $1 \mathrm{O}$ radial modes. The fundamental mode is dominant in these stars. Sections 7.2 and 8 .

11. No evidence of a resonance-induced systematic deformation of the light curves of Blazhko RRd stars is found, even in the case of V13, where the modulations of the F and 10 modes have the same periods and show antiphase amplitude changes similar to 10/2O Cepheids. Section 8.

12. The M3 data show that the "feedback effect" (Smolec et al. 2015a) between the mean brightness and the amplitude of the dominant mode in Blazhko RRd stars does not exist in general. The connection between the amplitude variations of the modes and the mean brightness (defined as the intensity-averaged mean $V$ magnitude) is different star by star. Section 8 .

13. Large period jumps are recorded in V13, V44, and V79, which are connected to the switch from fundamental- to double-mode pulsation. The value of the frequency increase equals with the modulation frequency (or its half), and a strong amplitude decrease accompanies the appearance of the overtone mode in Blazhko RRab stars. Different components of the Blazhko multiplets seem to be the highest amplitude signals at the fundamental-mode frequency in single- and double-mode stages in these stars. Sections 7.2 and 8. 
14. The period-ratio anomaly of Blazhko RRd stars cannot be resolved with any pair of the components of the multiplets appearing at the radial modes. Section 8.

As a conclusion, we would like to put into the focus the strange $f_{0.61}$ signals detected in different samples of classical radial pulsating stars and the special anomalous period ratios of RRd stars observed in this M3 survey as open questions requiring further investigation.

The authors would like to express their acknowledgment to the referee for the notes, questions, and comments, which helped us improve the clarity and quality of the paper. G.H. acknowledges support by the Ministry for the Economy, Development, and Tourisms Programa Iniciativa Científica Milenio through grant IC210009, awarded to the Millennium Institute of Astrophysics; by Proyecto Basal PFB-06/2007; by Fondecyt grant 1141141 ; and by grants CONICYT Anillo ACT1101 CONICYT-PCHA/Doctorado Nacional/201463140099. K.K. is supported by a Marie Curie International Outgoing Fellowship within the 7th European Community Framework Programme (PIOF 255267 SAS-RRL). N.J. acknowledges the OTKA K-83790 grant; M.A. the grant of the MTA CSFK Lendület Disk Research Group; and A.P. the LP2012-31, K-109276, and K-104607 grants. K.S. has been supported by the Lendület-2009 program of the HAS, the Hungarian OTKA grant K-104607, and the ESA PECS Contract No. 4000110889/14/NL/NDe.

\section{REFERENCES}

Alard, C. 2000, A\&AS, 144, 363

Benkő, J. M., Bakos, G. Á, \& Nuspl, J. 2006, MNRAS, 372, 1657

Benkő, J. M., Plachy, E., Szabó, R., Molnár, L., \& Kolláth, Z. 2014, ApJS, 213,31

Buchler, R., \& Kolláth, Z. 2011, ApJ, 731, 24

Cacciari, C., Corwin, T. M., \& Carney, B. W. 2005, AJ, 129, 267

Castelli, F., \& Kurucz, R. L. 2003, in IAU Symp. 210, Modelling of Stellar Atmospheres, ed. N. Piskunov, W.-W. Weiss, \& D.-F. Gray (San Francisco, CA: ASP), 20

Clement, C. M., \& Goranskij, V. P. 1999, ApJ, 513, 767

Clement, C. M., Hilditch, R. D., Kaluzny, J., \& Rucinski, S. M. 1997, ApJL, 489, L55

Clement, C. M., Muzzin, A., Dufton, Q., et al. 2001, AJ, 122, 2587

Clement, C. M., \& Thompson, M. 2007, JAVSO, 35, 336

Clementini, G., Corwin, T. M., Carney, B. W., \& Sumerel, A. N. 2004, AJ, 127,938

Corwin, B. W., \& Carney, B. W. 2001, AJ, 122, 3183

Cseresnjes, P. 2001, A\&A, 375, 909

Dziembowski, W. A. 2012, AcA, 62, 323

Garrido, R. 2004, in Second Eddington Workshop: Stellar Structure and Habitable Planet Finding, ed. F. Favata, S. Aigrain, \& A. Wilson ( ESA SP538; Noordwijk: ESA), 231
Gillet, D., \& Fokin, A. B. 2014, A\&A, 565, 73

Goranskij, V. P., \& Barsukova, E. A. 2007, ATel, 1120, 1

Goranskij, V. P., Clement, C. M., \& Thompson, M. 2010 (in Variable Stars, the Galactic halo and Galaxy Formation,) ed. C. Sterken, N. Samus, \& L. Szabados (Moscow: Sternberg Astronomical Institute of Moscow Univ.), 115

Govea, J., Gomez, T., Preston, G. W., \& Sneden, C. 2014, ApJ, 782, 59

Hartman, J. D., Kaluzny, J., Szentgyörgyi, A., \& Stanek, K. Z. 2005, AJ, 129,1596

Hurdis, D. A., \& Krajci, T. 2012, JAAVSO, 40, 268

Jurcsik, J. 2009, in AIP Conf. Proc. 1170, Stellar Pulsation: Challenges for Theory and Observation, ed. J. A. Guzik, \& P. A. Bradley (Melville, NY: AIP), 286

Jurcsik, J., Hajdu, G., Szeidl, B., et al. 2012a, MNRAS, 419, 2173

Jurcsik, J., Sódor, Á, Szeidl, B., et al. 2008, MNRAS, 393, 1553

Jurcsik, J., Sódor, Á, Szeidl, B., et al. 2009, MNRAS, 400, 1006

Jurcsik, J., Szeidl, B., Clement, C., Hurta, Z., \& Lovas, M. 2011, MNRAS, 411,1763

Jurcsik, J., Sódor, Á, Hajdu, G., et al. 2012b, MNRAS, 423, 993

Jurcsik, J., Smitola, P., Hajdu, G., \& Nuspl, J. 2014, ApJL, 797, L3

Kaluzny, J., Hilditch, R. W., Clement, C., \& Rucinski, S. M. 1998, MNRAS, 296, 347

Kolláth, Z. 1990, Occ. Techn. Notes Konkoly Obs No, 1 http://www.konkoly. hu/staff/kollath/mufran.html

Kolláth, Z., Molnár, L., \& Szabó, R. 2011, MNRAS, 414, 1111

Le Borgne, J.-F., Klotz, A., Poretti, E., et al. 2012, AJ, 144, 39

Marconi, M., \& Degl'Innocenti, S. 2007, A\&A, 474, 557

McClusky, J. V. 2008, IBVS, 5825, 1

Moskalik, P. 2014, in Proc. IAU Symp. 301, Precision Asteroseismology, ed. J. A. Guzik et al. (Cambridge: Cambridge Unvi. Press), 249

Moskalik, P., \& Kołaczkowski, Z. 2008, CoAst, 157, 343

Moskalik, P., \& Kołaczkowski, Z. 2009, MNRAS, 394, 1649

Moskalik, P., Smolec, R., Kolenberg, K., et al. 2015, MNRAS, 447, 2348

Munari, U., Sordo, R., Castelli, F., \& Zwitter, T. 2005, A\&A, 442, 1127

Netzel, H., Smolec, R., \& Moskalik, P. 2015, MNRAS, 447, 1173

Peterson, R. C., Rood, R. T., \& Crocker, D. A. 1995, ApJ, 453, 214

Preston, G. W., \& Chadid, M. 2013, in New Advances in Stellar Physics: From Microscopic to Macroscopic Processes, ed. G. Alecian et al. (EAS Pub. Ser. 63: Les Ulis: EDP Sciences), 35

Samus, N. N., Kazarovets, E. V., Pastukhova, E. N., Tsvetkova, T. M., \& Durlevich, O. V. 2009, PASP, 121, 1378

Sódor, Á 2012, Occ. Techn. Notes Konkoly Obs No., 1 www.konkoly.hu/staff/ sodor/lcfit.html

Sódor, Á, Jurcsik, J., Szeidl, B., et al. 2011, MNRAS, 411, 1585

Sódor, Á, Jurcsik, J., \& Szeidl, B. 2009, MNRAS, 394, 261

Smolec, R., Soszyński, I., Udalski, A., et al. 2015a, MNRAS, 447, 3756

Smolec, R., Soszyński, I., Udalski, A., et al. 2015b, MNRAS, 447, 3873

Soszyński, I., Poleski, R., Udalski, A., et al. 2008, AcA, 58, 163

Soszyński, I., Poleski, R., Udalski, A., et al. 2010, AcA, 60, 17

Soszyński, I., Dziembowski, W. A., Udalski, A., et al. 2011, AcA, 61, 1

Soszyński, I., Dziembowski, W. A., Udalski, A., et al. 2014, AcA, 64, 1

Stetson, P. B. 2000, PASP, 112, 925

Szabó, R. 2014, in Proc. of the Int. Astronomical Union, IAU Symposium 301, Precision Asteroseismology, ed. J. A. Guzik et al. (Cambridge: Cambridge Unvi. Press), 241

Szabó, R., Benkő, J. M., Paparó, M., et al. 2014, A\&A, 570, 100

Szentgyörgyi, A., Fúrész, G., Cheimets, P., et al. 2011, PASP, 123, 1188

Walker, A. R., \& Nemec, J. M. 1996, AJ, 112, 2026

Wils, P. 2010, IBVS, 5955, 1 